\title{
The elliptical galaxies NGC 1052 and NGC 7796
}

\section{Stellar populations and abundance ratio $\alpha / \mathrm{Fe}^{\star}$}

\author{
A. de C. Milone ${ }^{1}$, M. G. Rickes ${ }^{2}$, and M. G. Pastoriza ${ }^{2}$ \\ ${ }^{1}$ Instituto Nacional de Pesquisas Espaciais (INPE), Coordenação de Cências Espaciais e Atmosféricas, Divisão de Astrofísica, \\ Av. dos Astronautas 1758, São José dos Campos, SP 12227-010, Brazil \\ e-mail: acmilone@das.inpe.br \\ 2 Universidade Federal do Rio Grande do Sul (UFRGS), Instituto de Física, Departamento de Astronomia, Av. Bento Gonçalves \\ 9500, Porto Alegre, RS 91501-970, Brazil
}

Received 30 November 2005 / Accepted 22 March 2007

\begin{abstract}
Context. Understanding how each early-type galaxy forms and evolves is one of the objectives of extragalactic astrophysics and cosmology. The spatial distribution of the stellar populations inside a spheroidal system and their kinematic properties supply important information about the formation process. The reconstruction of the star formation history is crucial in this context.

Aims. We have performed a detailed stellar population analysis using long slit spectroscopic observations up to almost one effective radius of two different early-type galaxies of low density regions of the local Universe: NGC 1052, an E4 Liner prototype of a loose group that has a stellar rotating disc, and NGC 7796, an E1 of the field which shows a kinematically distinct core. Their mean luminosity-weighted stellar age, metallicity, and $\alpha / \mathrm{Fe}$ ratio along both photometric axes have been obtained to reconstruct the star formation history in their kinematically distinct subsystems.

Methods. We have measured Lick indices and computed their radial gradients. They were compared with the predicted ones of simple stellar population models. We have also applied a stellar population synthesis.

Results. The star characteristics are associated with their kinematics: they are older and $\alpha$-enhanced in the bulge of NGC 1052 and core of NGC 7796, while they show a strong spread of $\alpha / \mathrm{Fe}$ and age along the disc of NGC 1052 and an outward radial decrease outside the core of NGC 7796. The age variation is possibly connected to the $\alpha / \mathrm{Fe}$ one.

Conclusions. Both galaxies were formed by processes in which the star formation occurred first at the bulge (NGC 1052) and nucleus (NGC 7796) 12-15 Gyr ago on short timescales (0.1-1 Gyr), providing an efficient chemical enrichment by SN-II. In the disc of NGC 1052, there is some spread of age and formation timescales around its stars. In NGC 7796, the star formation timescale had some outward radial increases along both axes.
\end{abstract}

Key words. galaxies: elliptical and lenticular, cD - galaxies: stellar content - galaxies: individual: NGC 1052 galaxies: individual: NGC 7796

\section{Introduction}

Much effort has been spent to understand how stellar populations are formed and evolve in early-type galaxies. Early-type galaxies (or their nuclei) are not composed of a single-aged stellar population but a mix of simple stellar populations. Recent discoveries show that ellipticals are not merely a one-parameter family as a function of the global initial mass only (Yoshii \& Arimoto 1987). It is known they are a two-parameter family, given by the fundamental plane. Many ellipticals have signatures of interaction with the environment. These galaxies might have had different star formation histories, with stellar populations differing in metallicity and/or age (Worthey et al. 1992). Crucial information on the above issues have been compiled from the radial gradients of the metal line-strength indices for field ellipticals (Davies et al. 1993; Kobayashi \& Arimoto 1999) and for cluster ones (Jorgensen 1997; Mehlert et al. 2003). The metal linestrength gradients in early-type galaxies, for example, can vary

* Based on observations made in Observatório do Pico dos Dias (OPD), which is operated by LNA (Laboratório Nacional de Astrofísica), Brazil. considerably, ranging from essentially featureless to structured profiles showing e.g. changes of slope possibly associated with kinematically decoupled cores (Bender \& Surma 1992; Morelli et al. 2004), or anomalies in the stellar populations (Carollo \& Danziger 1994).

More recent studies about the stellar content of early-type galaxies have analyzed two dimensional spectroscopic data of their central parts in terms of kinematics and population parameters (e.g. Davies et al. 2001; McDermid et al. 2006). Davies et al. (2001) have found two independent kinematic spatial subsystems in the E3 galaxy NGC 4365 which have the same luminosity-weighted age (nearly 14 Gyr) and the same overabundance for the magnesium-to-iron ratio, indicating a common star formation history for both kinematically distinct components. McDermid et al. (2006) showed a summary of the results of two integral-field spectroscopic surveys using two instruments (SAURON and OASIS). These results led to an analysis in detail of the relationship between kinematically distinct components and their host galaxies in terms of the stellar age distribution (presence or absence of young populations). 
The metallicity radial gradients in bulges or early-type galaxies are related to the formation process of these spheroidal systems. A monolithic dissipative collapse of gas clouds associated with intense star formation can form a strong stellar metallicity gradient (Carlberg 1984; Chiosi \& Carraro 2002; Kawata 1999). On the other hand, it can also be induced by a hierarchical merging (Mihos \& Hernsquist 1994; Bekki \& Shioya 1999). Kobayashi (2004), in a general point of view, concludes that the metallicity and age gradients are dependent on the galaxy merging history. Forbes et al. (2005) found there is a direct correlation between the strength of the metallicity gradient and the mass of the early-types.

Many galaxies show in the central region a $\mathrm{Mg} / \mathrm{Fe}$ stellar abundance ratio larger than the solar one (i.e. an overabundance of the alpha-elements relative to the iron peak elements) which is interpreted as a consequence of the chemical enrichment given by type II supernovae relative to type Ia ones (Idiart et al. 2003). However, calcium is found underabundant relative to iron in ellipticals, through the analysis of different absorption lines of it, despite it being an alpha-element like magnesium: the Ca II triplet at $8600 \AA$ (Saglia et al. 2002), and the blue Lick index Ca4227 (Thomas et al. 2003b).

Moreover, the radial gradients of $\mathrm{Mg}$ line-strength indices of the Lick System can have equal or different behavior to those of Fe indices due to the galaxy formation process and the time scales of the star formation events.

Other studies have shown that the radial dependency of stel$\operatorname{lar} \alpha / \mathrm{Fe}$, or specifically the $\mathrm{Mg} / \mathrm{Fe}$ abundance ratio, can solve the star formation history inside a galaxy. Pipino et al. (2006) have found that NGC 4697 was formed by an outside-in process because the $\mathrm{Mg} / \mathrm{Fe}$ ratio is increasing outwards in that galaxy based on a chemical evolution analysis. However, the majority of the stellar population studies in ellipticals have shown that the oversolar $\mathrm{Mg} / \mathrm{Fe}$ abundance ratio is a global characteristic of an E galaxy (e.g. Mehlert et al. 2003). It is still not obvious that the $\mathrm{Mg} / \mathrm{Fe}$ abundance ratio is correlated to the global mass and/or the central velocity dispersion of the early-type galaxies (e.g. Henry \& Worthey 1999; Thomas et al. 2005).

At least three kinds of chemical evolution models have been proposed for ellipticals based on the occurrence of a internal galactic wind which causes the end of the star formation (Matteucci et al. 1998). During a long or short star burst, some amount of kinetical and thermal energy is transferred to the interstellar medium by the supernova explosions. When this energy is greater than the gravitational energy of the system, the interstellar material can be driven out of the galaxy by a global wind, which is produced by the supernovae. Thus the wind interrupts the star formation and the stellar populations evolve passively. One model is the classic wind model (Larson 1974a,b; Arimoto \& Yoshii 1987; Matteucci \& Tornambè 1987), which says that the star formation stops later in more massive galaxies than less massive ones because of their deeper gravitational potential wells. The consequence is to provide higher $\mathrm{Mg} / \mathrm{Fe}$ abundance ratios in less massive galaxies (disagreeing with the observed ratios). The other is the inverse wind model (Matteucci 1994), which establishes that the galactic wind occurs earlier in more massive galaxies providing an efficient star formation and a higher $\mathrm{Mg} / \mathrm{Fe}$ ratio. This is according to the relation $\mathrm{Mg}_{2}-\sigma_{v}^{0}$ (or $\log \langle\mathrm{Fe}\rangle-\sigma_{v}^{0}$ that has a weaker positive correlation). These chemical evolution models are based on the monolithic collapse of gas cloud. The last model was also proposed by Matteucci (1994). It suggests that more massive ellipticals are formed by merging of proto-clouds providing higher relative velocities to the cloudcloud collisions. The result is more star formation effectiveness for the more massive galaxies so that galactic wind can happen earlier providing more $\mathrm{Mg}$ enrichment relative to $\mathrm{Fe}$.

We have selected for the analysis of stellar populations two elliptical galaxies with intermediate masses $\left(\sim 10^{11} M_{\odot}\right)$ of low density regions of the local Universe: NGC 1052, which belongs to a loose group, and NGC 7796 to the field. They are different in terms of stellar kinematics: NGC 1052 has a stellar rotating disc and NGC 7796 a kinematically distinct core. Some parameters of them are given in Table 1.

The E4 galaxy NGC 1052 is well studied for several aspects: it is classified as a LINER prototype, and it has a nuclear jet at radio, optical and X-ray frequencies (Kadler et al. 2004). This elliptical has neutral hydrogen too. It is the third brightest member of a group with 11 galaxies identified by Giuricin et al. (2000). At $V, R$ and $I$ bands the isophotes, that follow de Vaucouleurs profile, change the ellipticity outwards from 0.10 up to 0.35 and they have an small isophotal twist too (Bender et al. 1988). Radial gradients of the $(U-R)$ and $(B-R)$ colours were observed in this galaxy (Peletier et al. 1990). The presence of nuclear gas was detected through HST Nicmos observations by Ravindranath et al. (2001). The NGC 1052 internal kinematics was analyzed by several authors. Binney et al. (1990), using dynamical models and photometric/spectroscopic data, have concluded that this elliptical has a rotating disc with inclination angle $i=90^{\circ}$ (a pure axisymmetric component) and it shows $M / L=4.5 h_{50}$ in $R$ band and $8.0 h_{50}$ in $B$ band. Fried \& Illingworth (1994) have calculated its rotation parameter $\left(V_{\text {rot }}^{\max } / \sigma_{v}^{0}\right)^{\star}=0.83 \pm 0.05$ and the logarithmic velocity dispersion radial gradient $\frac{\Delta \log \left(\sigma_{v}\right)}{\Delta \log (r)}=-0.010 \pm 0.020$. The Lick index $\mathrm{Mg}_{2}$ radial gradients along both photometric axes were firstly determined by Couture \& Hardy (1988). Carollo et al. (1993) have measured the gradients of some Lick indices along the E-W direction only. Central values of several Lick indices of NGC 1052 were obtained in some works (e.g. Trager et al. 1998; Beuing et al. 2002; and Thomas et al. 2005) but the majority have only published the central $\mathrm{Mg}_{2}$ (e.g. Terlevich et al. 1981, who were the first ones). The stellar population analysis of NGC 1052 made by Raimann et al. (2001), using long slit spectroscopic data over one direction only, indicates a larger spatial spread in age than in metallicity so that the old metal-rich populations dominate the nucleus but the young population of $1 \mathrm{Gyr}$ becomes important outside of the nuclear region. Thomas et al. (2005) have derived stellar age, $[\mathrm{Z} / \mathrm{H}],[\alpha / \mathrm{Fe}]$ for an extended sample of early-type galaxy nuclei. For NGC 1052, these parameters are: $21.7 \mathrm{Gyr},[\mathrm{Z} / \mathrm{H}]=+0.222$ and $[\alpha / \mathrm{Fe}]=+0.390 \mathrm{dex}$. On the other hand, the recent study of Pierce et al. (2005) of the stellar ages, metallicities and abundances ratios of the region inside $0.3 r_{\mathrm{e}}$ of NGC 1052 and a sample of 16 globular clusters of it have found that its nucleus has a luminosity-weighted age $\sim 2 \mathrm{Gyr}$ and $[\mathrm{Fe} / \mathrm{H}] \sim+0.6$ dex. They have not found any strong radial gradients in either age and metallicity, but there is a strong gradient in $\alpha$-element abundance whose central value is very high. Moreover, for all observed globular clusters of NGC 1052, they have obtained an age of $\sim 13 \mathrm{Gyr}$. These results indicate the NGC 1052 is a result of a recent merger (with starburst) that did not induce the formation of a young population of globular clusters. Apparently, it is a puzzle for the evolutionary history of this LINER elliptical galaxy.

NGC 7796 is an isolated E+ galaxy, or E1 using the ellipticity at $25 \mathrm{mag} \operatorname{arcmin}^{-2}$. According to $J H K_{\mathrm{s}}$ surface photometry of Rembold et al. (2002), this galaxy shows boxy isophotes with constant ellipticity and position angle. Its surface brightness profile follows the $r^{1 / 4}$ law with a decreasing value of $r_{\mathrm{e}}$ 
from $16.3 \operatorname{arcsec}\left(J\right.$ band) to $10.7 \operatorname{arcsec}\left(K_{\mathrm{s}}\right.$ band). According to Ferrari et al. (2002), NGC 7796 presents warm and cold dust components with uniform spherical distributions inside the central region. A counter rotating core for the stellar component was detected by Bertin et al. (1994). In addition, the stellar velocity dispersion along the major photometric axis changes from $230 \mathrm{~km} \mathrm{~s}^{-1}$ at the $r=20 \operatorname{arcsec}$ up to $270 \mathrm{~km} \mathrm{~s}^{-1}$ at the center. The radial profile of $\mathrm{Mg}_{2}$ was also observed by Bertin et al. (1994) and central values of several Lick indices were only obtained by Beuing et al. (2002) and Thomas et al. (2005). The stellar population for the central region was estimated having an age of $11.8 \mathrm{Gyr},[\mathrm{Z} / \mathrm{H}]=+0.248 \mathrm{dex}$ and $[\alpha / \mathrm{Fe}]=+0.344 \mathrm{dex}$ (Thomas et al. 2005).

The goal of this paper is to study in detail the radial distribution of the stellar populations along the main photometric directions in two different ellipticals, a LINER of a group NGC 1052 and the field galaxy NGC 7796, in order to understand the formation/evolution process of these galaxies, specially the star formation histories. The paper is organized as follows: Sect. 2 presents the observations, data reduction and kinematical measurements; Sect. 3 gives the Lick index measurements; Sect. 4 shows the radial gradients of some Lick indices; Sect. 5 presents the comparisons with the simple stellar population models; Sect. 6 describes the stellar population synthesis approach and Sect. 7 gives the star formation histories of these galaxies. In Sect. 8 a general discussion of the results is given and conclusions are drawn.

\section{Long slit spectroscopic observations, reductions and kinematical measurements}

Long slit spectroscopic observations of NGC1052 and NGC 7796 along their major and minor photometric axes were carried out on 1999 (August 12th and October 11th nights) at the Cassegrain focus with a Boller \& Chivens spectrograph of the $1.60 \mathrm{~m}$ telescope of the Observatório do Pico dos Dias (OPD) operated by the Laboratório Nacional de Astrofísica (LNA). The photometric parameters of NGC 1052 and NGC 7796 taken from the RC3 Catalogue and the extra-galactic data base NED are listed in Table $1 ; H_{0}=75 \mathrm{~km} \mathrm{~s}^{-1} \mathrm{Mpc}^{-1}$ has been adopted.

The slit width was 2.08 arcsec and its length was 230 arcsec. The spatial angular scale was $1.092 \operatorname{arcsec~pixel}^{-1}$. Adopting $h_{0}=0.75$, this corresponds to the linear scale of $111 \mathrm{pc} \mathrm{pixel}^{-1}$ for NGC 1052 ( $R$ distance of $1723 \mathrm{~km} \mathrm{~s}^{-1}$ from Faber et al. 1989) and $213 \mathrm{pc} \mathrm{pixel}^{-1}$ for NGC 7796 ( $c z_{\text {helio }}$ as distance indicator, Table 1$)$. The average seeing was $F W H M_{\text {seeing }}=2.0$ arcsec.

The spectral range at the wavelength of rest is $\lambda \lambda 4320-$ $6360 \AA$ and the sampling is $2.01 \AA$ pixel $^{-1}$ using a grating of 600 lines $\mathrm{mm}^{-1}$. The mean instrumental spectral resolution at $\lambda \lambda 4400-6200 \AA$ has $\sigma_{\text {inst }}=71 \mathrm{~km} \mathrm{~s}^{-1}$.

The total galaxy spectral exposures were divided in three equal sets of $30 \mathrm{~min}$ each for the major axis observations and $2 \times 30 \mathrm{~min}$ for the minor axis ones in order to perform the "cosmic ray" elimination and to obtain better signal-to-noise ratio for their aperture spectra.

Spectra of one $\mathrm{G}$ and seven $\mathrm{K}$ giants of the Lick sample (Worthey 1994 and http: //astro.wsu.edu/worthey/) were collected to calibrate the absorption line-strengths to the Lick System. These stellar spectra were also used to perform the cross correlations with the galaxy spectra following the Tonry \& Davis (1979) method. Spectrophotometric standard stars were observed as well.
Table 1. Data obtained in the RC3 Catalogue, the NASA/IPAC Extragalactic Database (NED) which is operated by the Jet Propulsion Laboratory, California Institute of Technology, under contract with the National Aeronautics and Space Administration and the extended version of the Lyon-Meudon Extragalactic DAtabase (the HyperLEDA) operated by the Centre de Recherche Astronomique de Lyon (for the mean values of the central velocity dispersion and $\mathrm{Mg}_{2}$ only).

\begin{tabular}{lrr}
\hline \hline Parameter & NGC 1052 & NGC 7796 \\
\hline Classifications & E4, LINER, Sy2 & E+ \\
PA & $120^{\circ}$ & $168^{\circ}$ \\
$\epsilon$ & 0.3082 & 0.1290 \\
$M_{B}$ & -20.50 & -20.79 \\
$B_{T}$ & 11.41 & 12.46 \\
$r_{\mathrm{e}}(\operatorname{arcsec})$ & 33.7 & 21.2 \\
$\mu_{\mathrm{e}}\left(\mathrm{mag} \mathrm{arcmin}^{-2}\right)$ & 12.15 & 12.20 \\
$r_{25}(\operatorname{arcsec})$ & 90.6 & 65.6 \\
$E(B-V)$ & 0.027 & 0.01 \\
$(B-V)_{\mathrm{e}}$ & 1.01 & 1.00 \\
$c z_{\text {helio }}\left(\mathrm{km} \mathrm{s}^{-1}\right)$ & $1510 \pm 6$ & $3290 \pm 24$ \\
Redshift $_{\left\langle\sigma_{v}^{0}\right\rangle\left(\mathrm{km} \mathrm{s}^{-1}\right)}$ & 0.00504 & 0.01097 \\
$\left\langle\mathrm{Mg}_{2}\right\rangle\left(\mathrm{mag}^{-1}\right)$ & $208 \pm 33$ & $259 \pm 11$ \\
Size $_{25}(\operatorname{arcmin} \times$ arcmin $)$ & $0.299 \pm 0.015$ & $0.237 \pm 0.003$ \\
Environment & $3.63 \times 2.51$ & $2.34 \times 2.04$ \\
\hline
\end{tabular}

\subsection{Data reduction, spectrum extraction and sky subtraction}

The digital images were processed and reduced using The NOAO Optical Astronomy Packages of IRAF. Firstly, the images were properly bias subtracted and flat field corrected. The cosmic ray hits above specific flux ratio thresholds were also removed in all of them.

The one-dimensional stellar spectra were extracted using apertures characterized by the FWHM of their profiles. The sky subtractions of them were made using two regions at 55 arcsec from the profile centers and a second order Chebyshev function to represent the sky level across the slit. These spectra were wavelength calibrated ( $\mathrm{rms}=0.2-0.4 \AA$ ) using the line identifications in the helium-argon frames which were obtained immediately before or after each exposure.

The aperture spectra along the major axis of NGC 1052 $\left(\mathrm{PA}=120^{\circ}\right)$ were extracted for the radial distances of $0.00,1.10$, $3.56,6.84,11.80,20.21$ and 37.03 arcsec each side of the center. They are adjacent to each other and each aperture is always greater than the previous one in order to improve the spectral quality. The symmetric spectral extractions along its minor axis $\left(\mathrm{PA}=210^{\circ}\right)$ were for the radial distances of $0.00,2.18,4.91$, 9.42 and 18.0 arcsec. The obtained spectral signal-to-noise ratio per Angstrom, $S / N\left(\AA^{-1}\right)$ has been from 9 (minor axis) and 36 (major axis) for the most external spectra up to 34 (minor axis) and 83 (major axis) for the central ones. The $S / N\left(\AA^{-1}\right)$ was measured in the spectral range $\lambda \lambda 5800-5850 \AA$ (wavelength of rest) according to the simple expression derived for the photon statistic by Cardiel et al. (1998).

The aperture spectra along the major axis of NGC 7796 $\left(\mathrm{PA}=168^{\circ}\right)$ were obtained for the radial distances of $0.00,1.10$, $3.55,7.10$ and 13.21 arcsec and along the minor axis for the distances of $0.00,1.09,3.55,6.93$ and 12.5 arcsec. The spectral $(S / N)\left(\AA^{-1}\right)$ was from 17 (minor axis) or 26 (major axis) for the most external spectra up to 47 (minor axis) or 61 (major axis) for the central ones.

The extractions of the long slit aperture spectra of both galaxies adopted second order Chebyshev functions to fit the background level across the slit at each wavelength. For 
Table 2. Parameters and stellar kinematical measurements of the long slit spectroscopic extraction regions along the major axis of NGC 1052.

\begin{tabular}{rrrrrr}
\hline \hline $\begin{array}{r}\text { Radius } \\
(\operatorname{arcsec})\end{array}$ & $\begin{array}{r}\text { Area } \\
\left(\operatorname{arcsec}^{2}\right)\end{array}$ & $\begin{array}{r}S / N \\
\left(\AA^{-1}\right)\end{array}$ & $\begin{array}{r}V_{\text {rot }} \\
\left(\mathrm{km} \mathrm{s}^{-1}\right)\end{array}$ & $\begin{array}{r}\sigma_{v} \\
\left(\mathrm{~km} \mathrm{~s}^{-1}\right)\end{array}$ & $R_{\mathrm{cc}}$ \\
\hline 0.00 & 4.53 & 83 & $0 \pm 18$ & $223 \pm 10$ & 18.5 \\
\hline $1.10 \mathrm{SE}$ & 4.53 & 78 & $-19 \pm 18$ & $221 \pm 09$ & 18.5 \\
$1.08 \mathrm{NW}$ & 4.53 & 78 & $+16 \pm 18$ & $225 \pm 09$ & 19.3 \\
\hline $3.56 \mathrm{SE}$ & 5.68 & 62 & $-36 \pm 16$ & $210 \pm 11$ & 22.2 \\
$3.54 \mathrm{NW}$ & 5.68 & 59 & $+44 \pm 16$ & $220 \pm 09$ & 22.2 \\
\hline $6.84 \mathrm{SE}$ & 7.95 & 48 & $-40 \pm 17$ & $211 \pm 14$ & 20.5 \\
$6.81 \mathrm{NW}$ & 7.95 & 45 & $+75 \pm 17$ & $216 \pm 14$ & 21.7 \\
\hline $11.80 \mathrm{SE}$ & 12.73 & 42 & $-60 \pm 18$ & $213 \pm 17$ & 18.7 \\
$11.78 \mathrm{NW}$ & 12.73 & 40 & $+79 \pm 17$ & $212 \pm 16$ & 20.6 \\
\hline $20.21 \mathrm{SE}$ & 22.26 & 36 & $-83 \pm 21$ & $212 \pm 18$ & 13.8 \\
$20.19 \mathrm{NW}$ & 22.26 & 36 & $+108 \pm 18$ & $182 \pm 16$ & 15.6 \\
\hline $37.03 \mathrm{SE}$ & 47.69 & 30 & $-105 \pm 30$ & $180 \pm 18$ & 7.2 \\
$37.01 \mathrm{NW}$ & 47.69 & 30 & $+149 \pm 30$ & $169 \pm 17$ & 7.2 \\
\hline
\end{tabular}

Notes. Column 1: distance of the extraction region to the galaxy center. SE and NW correspond to Southeast and Northwest respectively. Column 6: cross correlation factor $R_{\mathrm{cc}}$ of the best stellar template (HR 8924).

NGC 1052, the sky windows were localized from 81 arcsec of the galactic center. For NGC 7796, the sky windows stayed at $r \geq$ 67.5 arcsec for the major axis extractions and at $r \geq 55.7$ arcsec $\left(0.91 r_{25}^{\text {cor }}\right)$ for the minor axis ones. There were some flux residuals due to the poor subtraction of the telluric lines in the outer regions of the galaxies. However, these residuals are not in the spectrum windows of the Lick indices (except for Fe5406; see details in Sect. 3).

In the sky background windows, the contribution of the galaxy spectrum was smaller than $\approx 7.6 \%$ relative to the sky level in the OPD/LNA where $\left\langle V_{\text {sky }}\right\rangle=21.2 \mathrm{mag} \mathrm{arcsec}^{-2}$ in the dark nights of August/October on 1999. For the central aperture of an unique exposure of NGC 1052 (major axis), the relative error of the sky subtraction increases from $\sim 1.4 \%$ in the red up to $\sim 2.2 \%$ in the blue region. For a respective exposure of NGC 7796, we have obtained $\sim 1.9 \%$ in the red and $\sim 3.1 \%$ in the blue. For the most distant apertures of an exposure for NGC 1052 (major axis), this error is $\sim 6.4 \%$ in the red and $\sim 9.5 \%$ in the blue; and for an analog exposure of NGC 7796 , it is $\sim 5.7 \%$ in the red and $\sim 8.9 \%$ in the blue.

The relative difference between the sensitivity functions of distinct flux standard stars was $\leq 2.0 \%$ and $\leq 0.8 \%$ in the runs of August and October respectively. The influence of the flux calibration on the Lick index measurements is discussed in Sect. 3.

In summary, all aperture spectra of both galaxies were wavelength calibrated ( $\mathrm{rms} \leq 0.30 \AA$ ) using He-Ar frames (obtained immediately before or after of each exposure), accordingly summed, flux calibrated, deredden using the respective line-of-sight Galactic extinction, put at the rest wavelength using the observed recession velocities (from the cross correlation measurements) and flux normalized to $\lambda 5870 \AA$. The parameters and kinematical measurements of all long slit spectroscopic extractions are given in Tables 2 and 3 for NGC 1052 and Tables 4 and 5 for NGC 7796.

\subsection{Stellar kinematical measurements with external comparisons}

The line-of-sight rotation curve and the line-of-sight velocity dispersion radial profile of the stellar component along both photometric axes were measured for both galaxies using our
Table 3. Parameters and stellar kinematical measurements of the long slit spectroscopic extraction regions along the minor axis of NGC 1052.

\begin{tabular}{rrrrrr}
\hline \hline $\begin{array}{r}\text { Radius } \\
(\operatorname{arcsec})\end{array}$ & $\begin{array}{r}\text { Area } \\
\left(\operatorname{arcsec}^{2}\right)\end{array}$ & $\begin{array}{r}S / N \\
\left(\AA^{-1}\right)\end{array}$ & $\begin{array}{r}V_{\text {rot }} \\
\left(\mathrm{km} \mathrm{s}^{-1}\right)\end{array}$ & $\begin{array}{r}\sigma_{v} \\
\left(\mathrm{~km} \mathrm{~s}^{-1}\right)\end{array}$ & $R_{\mathrm{cc}}$ \\
\hline 0.00 & 4.53 & 34 & $0 \pm 18$ & $236 \pm 24$ & 17.1 \\
\hline $2.17 \mathrm{SW}$ & 4.53 & 25 & $+11 \pm 18$ & $234 \pm 32$ & 18.0 \\
$2.19 \mathrm{NE}$ & 4.53 & 24 & $-21 \pm 20$ & $233 \pm 32$ & 15.0 \\
\hline $4.90 \mathrm{SW}$ & 6.82 & 17 & $+8 \pm 18$ & $214 \pm 33$ & 17.7 \\
$4.92 \mathrm{NE}$ & 6.82 & 16 & $+1 \pm 19$ & $220 \pm 37$ & 15.6 \\
\hline $9.41 \mathrm{SW}$ & 11.92 & 12 & $-5 \pm 21$ & $233 \pm 47$ & 13.7 \\
$9.43 \mathrm{NE}$ & 11.92 & 12 & $+5 \pm 19$ & $177 \pm 25$ & 14.0 \\
\hline $18.00 \mathrm{SW}$ & 23.80 & 9 & $-6 \pm 29$ & $184 \pm 28$ & 7.5 \\
$18.01 \mathrm{NE}$ & 23.80 & 9 & $-10 \pm 29$ & $144 \pm 18$ & 6.6 \\
\hline
\end{tabular}

Notes. Column 1: SW and NE correspond to Southwest and Northeast respectively.

Column 6: as in Table 2.

Table 4. Parameters and stellar kinematical measurements of the long slit spectroscopic extraction regions along the major axis of NGC 7796.

\begin{tabular}{rrrrrr}
\hline \hline $\begin{array}{r}\text { Radius } \\
(\operatorname{arcsec})\end{array}$ & $\begin{array}{r}\text { Area } \\
\left(\operatorname{arcsec}^{2}\right)\end{array}$ & $\begin{array}{r}S / N \\
\left(\AA^{-1}\right)\end{array}$ & $\begin{array}{r}V_{\text {rot }} \\
\left(\mathrm{km} \mathrm{s}^{-1}\right)\end{array}$ & $\begin{array}{r}\sigma_{v} \\
\left(\mathrm{~km} \mathrm{~s}^{-1}\right)\end{array}$ & $R_{\mathrm{cc}}$ \\
\hline 0.00 & 4.53 & 61 & $0 \pm 14$ & $261 \pm 10$ & 26.6 \\
\hline $1.09 \mathrm{~S}$ & 4.53 & 56 & $-3 \pm 13$ & $257 \pm 11$ & 28.1 \\
$1.09 \mathrm{~N}$ & 4.53 & 56 & $+8 \pm 15$ & $274 \pm 14$ & 24.0 \\
\hline $3.55 \mathrm{~S}$ & 5.68 & 41 & $+4 \pm 16$ & $255 \pm 20$ & 21.9 \\
$3.55 \mathrm{~N}$ & 5.68 & 40 & $+1 \pm 14$ & $271 \pm 20$ & 25.4 \\
\hline $7.10 \mathrm{~S}$ & 9.09 & 32 & $+20 \pm 17$ & $237 \pm 26$ & 17.2 \\
$7.10 \mathrm{~N}$ & 9.09 & 31 & $-8 \pm 18$ & $260 \pm 30$ & 16.4 \\
\hline $13.21 \mathrm{~S}$ & 16.35 & 26 & $+50 \pm 24$ & $240 \pm 33$ & 10.6 \\
$13.21 \mathrm{~N}$ & 16.35 & 26 & $+25 \pm 24$ & $243 \pm 34$ & 10.9 \\
\hline
\end{tabular}

Notes. Column 1: $\mathrm{S}$ and $\mathrm{N}$ correspond to South and North respectively. Column 6: as in Table 2.

Table 5. Parameters and stellar kinematical measurements of the long slit spectroscopic extraction regions along the minor axis for NGC 7796.

\begin{tabular}{rrrrrr}
\hline \hline $\begin{array}{r}\text { Radius } \\
(\operatorname{arcsec})\end{array}$ & $\begin{array}{r}\text { Area } \\
\left(\operatorname{arcsec}^{2}\right)\end{array}$ & $\begin{array}{r}S / N \\
\left(\AA^{-1}\right)\end{array}$ & $\begin{array}{r}V_{\text {rot }} \\
\left(\mathrm{km} \mathrm{s}^{-1}\right)\end{array}$ & $\begin{array}{r}\sigma_{v} \\
\left(\mathrm{~km} \mathrm{~s}^{-1}\right)\end{array}$ & $R_{\mathrm{cc}}$ \\
\hline 0.00 & 4.53 & 47 & $0 \pm 14$ & $261 \pm 16$ & 24.7 \\
\hline $1.09 \mathrm{E}$ & 4.53 & 44 & $-2 \pm 15$ & $267 \pm 18$ & 23.9 \\
$1.09 \mathrm{~W}$ & 4.53 & 44 & $-1 \pm 15$ & $260 \pm 18$ & 24.4 \\
\hline $3.55 \mathrm{E}$ & 5.68 & 31 & $+12 \pm 16$ & $265 \pm 26$ & 19.5 \\
$3.55 \mathrm{~W}$ & 5.68 & 32 & $-8 \pm 16$ & $253 \pm 28$ & 20.3 \\
\hline $6.93 \mathrm{E}$ & 8.40 & 22 & $+18 \pm 21$ & $253 \pm 41$ & 13.1 \\
$6.93 \mathrm{~W}$ & 8.40 & 22 & $-35 \pm 21$ & $224 \pm 32$ & 12.3 \\
\hline $12.50 \mathrm{E}$ & 14.77 & 17 & $+28 \pm 34$ & $264 \pm 40$ & 7.3 \\
$12.50 \mathrm{~W}$ & 14.77 & 17 & $-23 \pm 23$ & $215 \pm 33$ & 10.6 \\
\hline
\end{tabular}

Notes. Column 1: E and W correspond to East and West respectively. Column 6: as in Table 2.

kinematical data: $c z_{\text {helio }}$ and $\sigma_{v}$ of the aperture spectra. We have also calculated the rotational parameter $\left(V_{\text {rot }}^{\max } / \sigma_{v}^{0}\right)^{\star}$, the kinematical misalignment $\Psi_{k}$ and the logarithmic radial gradient of the velocity dispersion $\frac{\Delta \log \sigma_{v}}{\Delta \log r}$ for both galaxies. Our data were compared with the literature data.

We adopted for this purpose the cross correlation method (Tonry \& Davies 1979) using the RVSAO (Radial Velocity Package of the Smithsonian Astrophysical Observatory) in IRAF enviroment (Kurtz \& Mink 1998). The uncertainty of the geocentric radial velocity of each aperture spectrum is computed by $\frac{3}{8} \frac{F W H M_{\text {peak }}}{(1+r)}$, where $F W H M_{\text {peak }}$ is the $F W H M$ of the peak of the 
cross correlation function, $r$ is the ratio between the height of this peak and the amplitude of a sinusoidal noise for the function (Kurtz \& Mink 1998). The heliocentric radial velocities of the stellar templates (K giants) came from the Duflot et al. (1995) Catalogue.

We had to apply an empirical calibration in order to transform the $F W H M_{\text {peak }}$ into the galaxy velocity dispersion as made for example by de la Rosa et al. (2001). This takes into account the instrumental resolution and closely obeys the relation $F W H M_{\text {peak }}=2 \sqrt{\ln 4} \times \sqrt{\sigma_{v}^{2}+2 \times \sigma_{\text {inst }}}$, where $\sigma_{\text {inst }}$ corresponds to the mean $\sigma_{\text {peak }}$ of the stellar auto-correlations divided by $\sqrt{2}$. A parabolic function fitting was adopted to fit the half of the height of the correlation peak. A third order polynomial function $F W H M_{\text {peak }}\left(\sigma_{v}\right)$ was obtained adopting correlations between artificially broadened spectra of six observed stars (HR 6136, HR 6159, HR 6299, HR 8841 and HR 8924) and the non-broadened stellar ones. The mean instrumental resolution $\sigma_{\text {inst }}=71 \pm 10 \mathrm{~km} \mathrm{~s}^{-1}(\lambda \lambda 4400-6200 \AA)$ was estimated by the template-template correlations. Gaussian convolutions were made to represent $\sigma_{v}$ of 50 up to $400 \mathrm{~km} \mathrm{~s}^{-1}$ with steps of $50 \mathrm{~km} \mathrm{~s}^{-1}$.

The velocity dispersion errors were estimated as a function of the signal-to-noise ratio and $\sigma_{v}$ itself. Six levels of artificial Poisson noise were applied to all artificially broadened spectra of HR 8924 in order to represent the $S / N$ variation of 14 up to $85 \AA^{-1}$. For each noisy broadened spectrum, the velocity dispersion was measured and compared with the actual value. After that, a second order polynomial function of the $S / N\left(\AA^{-1}\right)$ was constructed for each one of the $\sigma_{v}$ values (of 50 up to $400 \mathrm{~km} \mathrm{~s}^{-1}$ ) to estimate the relative error of the velocity dispersion. For example, the typical error of $\sigma_{v}=200 \mathrm{~km} \mathrm{~s}^{-1}$ varies from 4 up to $16 \%$ when the $S / N\left(\AA^{-1}\right)$ changes from 85 to $14 \AA^{-1}$.

The stellar kinematical results of all observed galaxy regions are shown in Tables 2 and 3 (NGC 1052) and 4 and 5 (NGC 7796), including the $R_{\mathrm{cc}}$ factor of the respective best cross correlation. Our measurements of $c z_{\text {helio }}$ of the central aperture are in agreement with the catalog values, i.e. the relative differences are smaller than $9 \%$ for NGC 1052 and $3.5 \%$ for NGC 7796.

Figures $1 \mathrm{a}$ and $1 \mathrm{~b}$ show respectively the rotation curve and the velocity dispersion radial profile of NGC 1052 along its major axis. Figures $2 \mathrm{a}$ and $2 \mathrm{~b}$ show the same its the minor axis. Our results are also plotted together with those of Binney et al. (1990) for both photometric directions and Fried \& Illingworth (1994) for the major axis only. Figures $3 a$ and $3 b$ show respectively the rotation curve and the velocity dispersion radial profile of NGC 7796 along its major photometric axis. Figures 4a and $4 \mathrm{~b}$ show the same for its minor axis. Our data are plotted together with those of Bertin et al. (1994) whose observations were made along the major axis only (Figs. 3a,b).

In order to compare our results about velocity dispersion with the literature, the observed $\sigma_{v}^{\text {obs }}$ of the central spectra must be corrected for the aperture effect considering a typical value for the logarithmic radial gradient of $\sigma_{v}$ (Jorgensen et al. 1995).

Taking into account the observations over both axes and adopting $\frac{\Delta \log \sigma_{v}}{\Delta \log r}=-0.040$ from Jorgensen et al. (1995), the mean values of $\sigma_{v}^{0}$ of NGC 1052 and NGC 7796 are $213 \pm 9 \mathrm{~km} \mathrm{~s}^{-1}$ and $250 \pm 8 \mathrm{~km} \mathrm{~s}^{-1}$ respectively. They are in excellent agreement with the published ones. For NGC 1052, LEDA presents $\sigma_{v}^{0}=208 \pm$ $33 \mathrm{~km} \mathrm{~s}^{-1}$ from 24 measurements including Binney et al. (1990) and Fried \& Illingworth (1994) who measured $240 \pm 10 \mathrm{~km} \mathrm{~s}^{-1}$ and $195 \pm 15 \mathrm{~km} \mathrm{~s}^{-1}$ respectively. For NGC 7796, LEDA shows $\sigma_{v}^{0}=259 \pm 11 \mathrm{~km} \mathrm{~s}^{-1}$ from two studies only; one of them
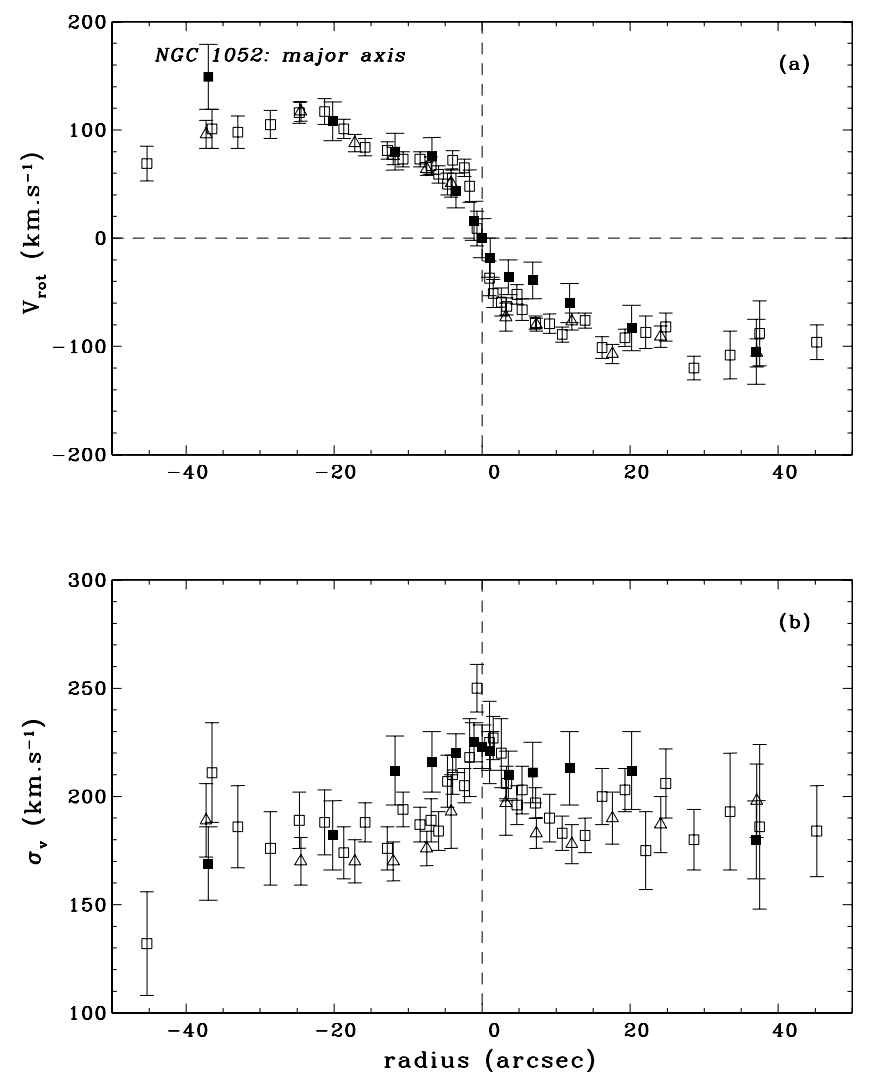

Fig. 1. The stellar kinematical measurements along the major axis of NGC 1052. a) The line-of-sight velocity rotation curve; b) the line-ofsight velocity dispersion radial profile. Our data are plotted using solid squares. The data of Binney et al. (1990), open squares, and Fried \& Illingworth (1994), open triangles, are presented as well.

is Bertin et al. (1994) who measured $265 \pm 9 \mathrm{~km} \mathrm{~s}^{-1}$. The individual $\sigma_{v}^{0}$ for NGC 1052 are: $207 \pm 10 \mathrm{~km} \mathrm{~s}^{-1}$ (major axis) and $220 \pm 24 \mathrm{~km} \mathrm{~s}^{-1}$ (minor axis). For NGC 7796 they are: $250 \pm 10 \mathrm{~km} \mathrm{~s}^{-1}$ (major axis) and $251 \pm 16 \mathrm{~km} \mathrm{~s}^{-1}$ (minor axis).

For NGC 1052, the rotation curve along the major axis is similar to those acquired by Binney et al. (1990) and Fried \& Illingworth (1994); see Fig. 1a. The rotation curve over the minor axis is in agreement with that of Binney et al. (1990); see Fig. 2a. Along the major axis, despite the present worse spatial resolution, our result shows nuclear stellar rotation suggesting a rigid body (up to $r=4-7$ arcsec with a projected angular velocity of $10 \mathrm{~km} \mathrm{~s}^{-1} \operatorname{arcsec}^{-1}$ ), a discontinuity region at $r=7-12$ arcsec in a plateau, and a similar rigid body rotation outwards after this rotation discontinuity with an smaller projected angular velocity $\left(\sim 2 \mathrm{~km} \mathrm{~s}^{-1} \operatorname{arcsec}^{-1}\right)$. However, our result reveal an increasing of $V_{\text {rot }}$ outside of the observed region, differently from the Binney et al. (1990) and Fried \& Illingworth (1994) data that both show a constant value. Along the minor axis, no residual rotation is detected for the stellar component. The present line-of-sight velocity dispersion radial profile along the major axis is similar to those of Binney et al. (1990) and Fried \& Illingworth (1994); see Fig. 1b. The $\sigma_{v}$ profile along the minor axis is in agreement with that of Binney et al. (1990): Fig. 2b. For the major axis profile, the main difference is that the data of Binney et al. (1990) and Fried \& Illingworth (1994) have a more negative $\sigma_{v}$ gradient (up to $r=10 \mathrm{arcsec}$ ) than our data. The calculated rotational parameter $\left(V_{\text {rot }}^{\max } / \sigma_{v}^{0}\right)^{\star}$ assumes the nuclear velocity dispersion $\sigma_{v}^{0}$ corrected for the aperture effect using the logarithmic $\sigma_{v}$ radial gradient measured along the major 

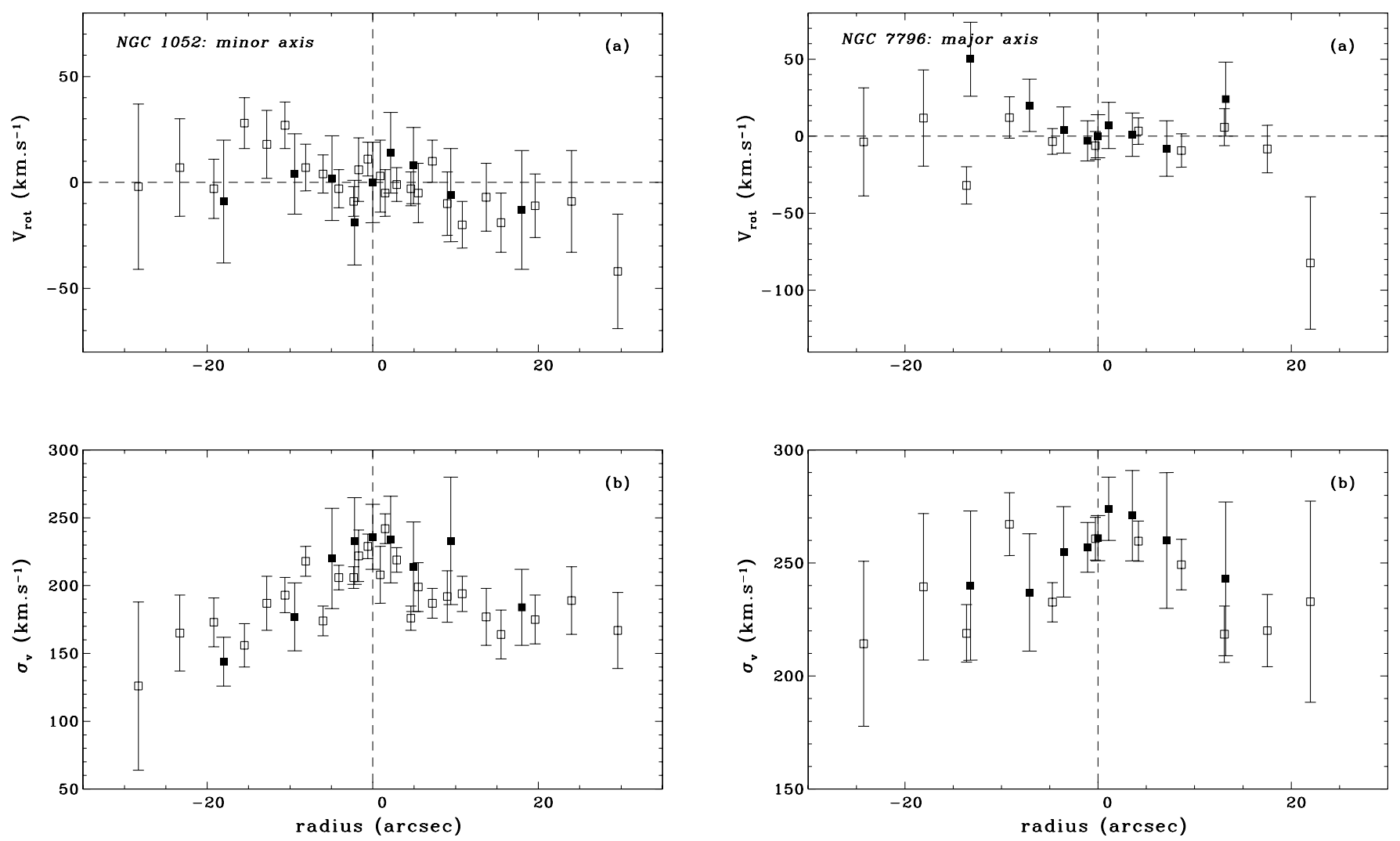

Fig. 2. The stellar kinematical measurements along the minor axis of NGC 1052. a) The line-of-sight velocity rotation curve; b) the line-ofsight velocity dispersion radial profile. Our data are plotted using solid squares. The data of Binney et al. (1990), open squares, are presented as well.

axis direction in the present work $\left(\frac{\Delta \log \sigma_{v}}{\Delta \log r}=-0.046 \pm 0.015\right)$. Our maximum line-of-sight rotational velocity $V_{\text {rot }}^{\max }$ is the average of those measured on each side of the $V_{\text {rot }}$ radial profile which were calculated using a third polynomial fit for the velocity curve that takes into account the errors in $V_{\text {rot }}$ only. NGC 1052 is a spheroidal system flattened by the rotational residual motion around the minor axis. Its rotational parameter, considering all the observed region $\left(\leq 3.8 \mathrm{kpc} \sim 0.9 r_{\mathrm{e}}\right)$, is $\left(V_{\mathrm{rot}}^{\max } / \sigma_{v}^{0}\right)^{\star}=0.89( \pm 0.14)$ which is in close agreement with the one found by Fried \& Illingworth (1994). Its maximum rotational velocity is $122 \pm 13 \mathrm{~km} \mathrm{~s}^{-1}$ in the observed region, but it still appears to increase outwards. Its nuclear velocity dispersion is $204 \pm 10 \mathrm{~km} \mathrm{~s}^{-1}$ (Coma normalized using its $\sigma_{v}$ radial gradient). Its line-of-sight velocity dispersion radial profile inside $0.9 r_{\mathrm{e}}$ is nearly symmetric. We could classify NGC 1052 as an oblate rotator, but NGC 1052 has a fast rotating stellar component because its rotational parameter does not reach unity. The estimated kinematic misalignment $\Psi_{\text {est }}$ is about $3^{\circ}$, if the maximum projected rotational velocity along the minor axis is not zero. This indicates that the stellar disc of NGC 1052 has an inclination angle very near to $90^{\circ}$ or, in other words, the photometric axes of this elliptical are direct projections of the kinematical axes. This corroborates the result of Binney et al. (1990).

For NGC 7796, the rotation curve and $\sigma_{v}$ radial profile, both along the major axis, are in agreement with those of Bertin et al. (1994) up to $r=14$ arcsec; see Figs. 3a,b. NGC 7796 has no significant stellar rotation around both axes. Its rotational parameter $\left(V_{\text {rot }}^{\max } / \sigma_{v}^{0}\right)^{\star}$ is $0.15( \pm 0.04)$. The velocity dispersion profiles inside the observed region $\left(\leq 4.3 \mathrm{kpc} \sim 0.6 r_{\mathrm{e}}\right)$

Fig. 3. The stellar kinematical measurements along the major axis of NGC 7796. a) The line-of-sight velocity rotation curve; b) the line-ofsight velocity dispersion radial profile. Our data are plotted using solid squares. The data of Bertin et al. (1994), open squares, are presented as well.

are nearly uniform; see Figs. $3 \mathrm{~b}$ and $4 \mathrm{~b}$. The logarithmic $\sigma_{v}$ radial gradient measured over the major axis in the present work is $\frac{\Delta \log \sigma_{v}}{\Delta \log r}=-0.028 \pm 0.030$. The nuclear velocity dispersion is $253 \pm 10 \mathrm{~km} \mathrm{~s}^{-1}$ (Coma normalized using this $\sigma_{v}$ gradient). Its $\Psi_{\text {est }}$ is very high, $\sim 62^{\circ}$. Bertin et al. (1994), whose long slit observations have greater spatial scale $\left(1.78 \operatorname{arcsec~pixel}^{-1}\right)$, have concluded that NGC 7769 has a counter rotating core inside $r=4$ arcsec that our observations marginally corroborate. But, if the major axis rotation curve is considered up to $r=8$ arcsec we would detect a kinematically decoupled inner component ( $r \leq 3.5$ arcsec) that has no rotation while "the main body" has a very small rotation $\left(14 \mathrm{~km} \mathrm{~s}^{-1}\right)$. The minor axis rotation curve (Fig. 4a), considered only up to $r=7$ arcsec, does not show any rotation inside $r=1.5$ arcsec and it presents a small rotation $\left(26 \mathrm{~km} \mathrm{~s}^{-1}\right)$ but greater than another one about the major axis. Thus, the core of NGC 7796 shows complex kinematics.

\section{Measurements of the Lick indices}

The Lick indices are measurements of optical absorption lines of spectra of stars and stellar composite systems like globular clusters and galaxies. They have been employed to quantity the luminosity-weighted mean ages, metallicities and abundance ratios (as $\alpha$-elements/Fe) of the composite stellar populations with ages $\geq 2$ Gyr. The Lick indices of atomic lines are measured as equivalent widths $E W$ ( $\mathrm{A}$ unity) considering a linear local pseudo-continuum, which is defined by two wavelength windows next to the central feature bandpass $\left[\lambda_{1}, \lambda_{2}\right]$. The Lick indices of molecular lines quantify the absorbed flux in the index 

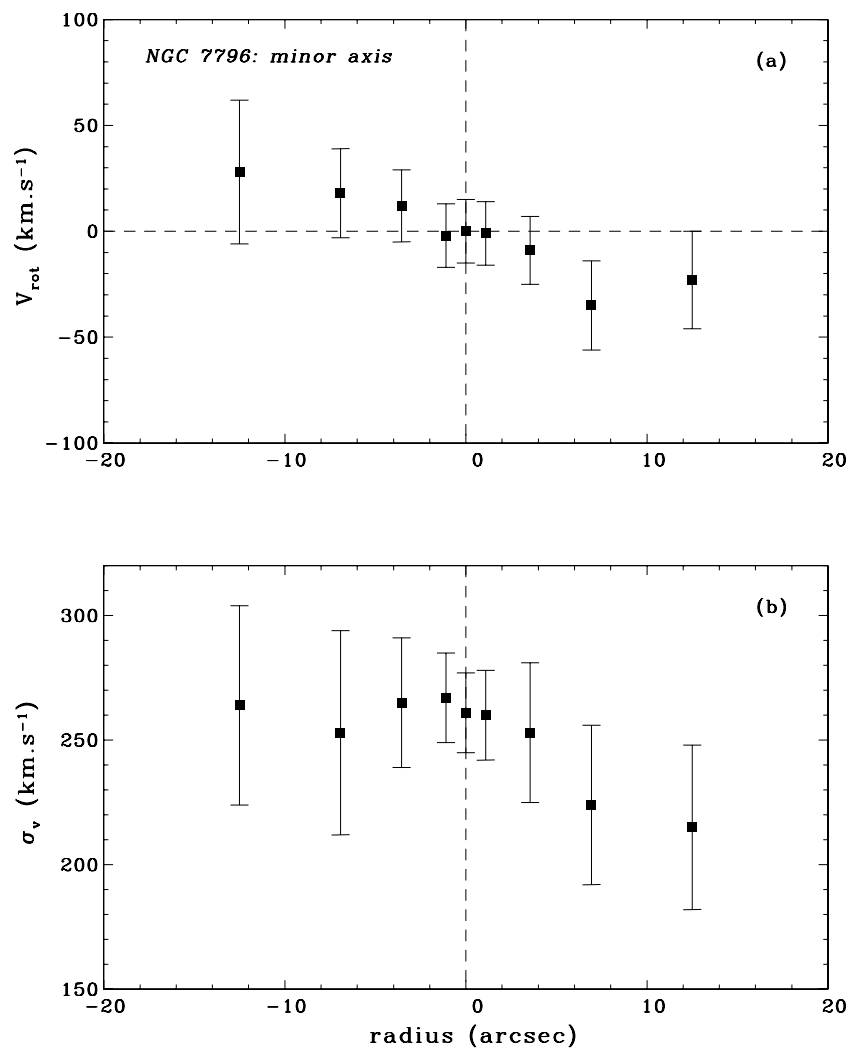

Fig. 4. The stellar kinematical measurements along the minor axis of NGC 7796. a) The line-of-sight velocity rotation curve; b) the line-ofsight velocity dispersion radial profile.

bandpass relative to the pseudo-continuum and it is expressed in the magnitude scale (here denoted by $M A G$ ). These linestrengths were originally measured in flux non-calibrated spectra with low resolution using an one-dimensional image detector (Image Digital Scanner, IDS, of the Lick Observatory). The spectral resolution of the Lick System is actually variable in the range $\lambda \lambda 4000-6000 \AA: F W H M_{\text {Lick }}=8.4-11.5 \AA$ with greater values in the red and blue edges (Worthey \& Ottaviani 1997). The Lick indices are defined to be independent of the line-ofsight velocity dispersion of the stellar system. Therefore, the spectral broadening effect due to the velocity dispersion must be subtracted in quadrature.

The EW indices can be transformed into the MAG index scale and vice-verse: $M A G=-2.5 \log \left[1-\frac{E W}{\left(\lambda_{2}-\lambda_{1}\right)}\right]$ and $E W=$ $\left(\lambda_{2}-\lambda_{1}\right)\left(1-10^{-0.4 M A G}\right)$.

\subsection{Lick calibration and index errors}

The current instrumental resolution $\sigma_{\text {inst }}$ is nearly $71 \mathrm{~km} \mathrm{~s}^{-1}$ considering the interval $\lambda \lambda 4400-6200 \AA$ so that $F W H M \sim$ $3 \AA$ at $\lambda 5300 \AA$. The respective one of the Lick System is $\sigma_{\text {Lick }}=220 \mathrm{~km} \mathrm{~s}^{-1}$, or $F W H M_{\text {Lick }} \approx 9.2 \AA$ in approximately the same range (Worthey \& Ottaviani 1997). Note that $\sigma\left(\mathrm{km} \mathrm{s}^{-1}\right)=$ $\frac{c . F W H M}{2 \sqrt{\ln 4} \lambda}$. Therefore, we had to put all galaxy and IDS sample star spectra to the Lick spectral resolution in order to measure the absorption line indices. All spectra were broadened using Gaussian fits. The appropriate spectral broadening $\sigma_{\text {broad }}$ was obtained from the result of the cross correlation of the actual Lick/IDS spectra (http://astro.wsu.edu/worthey/) with the observed stellar sample, subtracting the instrumental spectral resolution in quadrature, i.e. $\sigma_{\text {Lick }}^{2}=\sigma_{\text {inst }}^{2}+\sigma_{\text {broad }}^{2}$.

All galaxy and star spectra were accurately reduced to the wavelength of rest and broadened to the Lick resolution accordingly. The indices of Fe4383 to $\mathrm{NaD}$, including $\mathrm{Mg}_{1}$ and $\mathrm{Mg}_{2}$, were measured. However, two steps are still necessary in order to transform the index measurements to the Lick System: (i) a linear correction; and (ii) a correction for the line-of-sight velocity dispersion $\sigma_{v}$ (Worthey \& Ottaviani 1997). Both steps must be carefully done and they must be used to estimate the final errors of the indices Lick. It is still necessary to compare the indices to the published ones, specifically those for the galaxy nuclei. We have adopted eight Lick standard stars (see Sect. 2).

The first step is made through linear correlations between the actual published stellar Lick indices and the measured ones in our adequately broadened stellar spectra, like INDEX $_{\text {Lick }}=$ $\mathrm{A}+\mathrm{B} \times \mathrm{INDEX}_{\text {our }}$ (see Table 6 ). The angular coefficient stayed in the range $0.63 \leq B \leq 1.27$, and fitting rms was smaller than $0.37 \AA$ for the $E W$ indices excluding the Fe5015 index $(\mathrm{rms}=0.63 \AA)$ and $\leq 0.0062 \mathrm{mag}$ for the $M A G$ ones. The range of the index values measured in the broadened spectra of the eight Lick standards was similar to those ones observed in the galaxies, i.e. Fe4383 in [4.50, $8.50 \AA]$, Ca4455 in [0.50, $2.00 \AA]$, $\mathrm{Fe} 4531$ in $[3.00,4.50 \AA]$, Fe4668 in [5.00, $9.00 \AA ̊ \mathrm{~A}], \mathrm{H} \beta$ in $[0.50$, $2.10 \AA], \mathrm{Fe} 5015$ in $[5.00,7.00 \AA], \mathrm{Mgb}$ in $[2.00,5.00 \AA]$, Fe5270 in $[2.80,4.20 \AA], F e 5335$ in $[2.20,4.20 \AA], F e 5406$ in $[1.40,3.00 \AA], F e 5709$ in $[0.90,1.60 \AA]$, Fe5782 in [0.60, $1.50 \AA], \mathrm{NaD}$ in $[1.80,5.40 \AA], \mathrm{Mg}_{1}$ in [0.010, $\left.0.230 \mathrm{mag}\right]$, and $\mathrm{Mg}_{2}$ in [0.100, $\left.0.400 \mathrm{mag}\right]$.

The second step is based on the galaxy velocity dispersion that broadens the absorption lines of each spectrum. Except for the $\mathrm{H} \beta$ Lick index, the general consequence is a decreasing of the index value. We computed artificial broadenings of our Lick observed stellar spectra to different $\sigma_{\text {broad }}$ representative of the line-of-sight galaxy $\sigma_{v}$. Again, we have assumed spectral broadening by Gaussian convolutions for $\sigma_{v}$ from 50 to $400 \mathrm{~km} \mathrm{~s}^{-1}$ (with $50 \mathrm{~km} \mathrm{~s}^{-1}$ steps). The $F\left(\sigma_{v}\right)$ correction factor is the ratio of the measured index in the stellar observed spectrum to the measured index in the spectrum of a given representative $\sigma_{v}$ denoted by $\frac{E W(\text { index })_{0}}{E W(\text { index })_{\sigma_{\nu}}}$ where $E W(\text { index })_{0}$ is the equivalent width of the index for an unbroadened spectrum (i.e. $\sigma_{v}=0 \mathrm{~km} \mathrm{~s}^{-1}$ ). We have computed a second order polynomial fit in order to determine the mean correction factor as a function of the actual velocity dispersion for each Lick index, $\left.F\left(\sigma_{v}\right)=a+b \times \sigma_{v}+c \times \sigma_{v}^{2}\right)$; see Table 6 . The respective fitting rms was always smaller than $0.08 \AA$, except for the Ca4455 index $(0.14 \AA)$.

The final errors of the Lick indices for each aperture spectrum were computed considering a Poisson error of the respective measurement, the error of the computed heliocentric velocity and the error propagations due to both steps of the Lick transformation. The Poisson uncertainty of each index, $\delta(E W)$ or $\delta(\mathrm{MAG})$, was estimated as a function of the $S / N\left(\AA^{-1}\right)$ of each extracted spectrum following the simple expressions of Cardiel et al. (1998).

In Table 7, the errors of the Lick indices and their respective propagations due to the Lick calibration are shown for two extreme spectral $S / N\left(\AA^{-1}\right)$ ratios (83 from the central spectrum of NGC 1052 and 26 from the most external one of NGC 7796). For higher $S / N$, the final errors of the Lick indices are determinate by the Lick transformation and, for lower $S / N$, the intrinsic errors of the measurements become important to determine the final errors, except for Ca4455 and Fe5335. 
Table 6. Coefficients of the calibration to the Lick System: the linear correction, $\operatorname{INDEX}_{\text {Lick }}=A+B \times \operatorname{INDEX}_{\text {our }}$, and the velocity dispersion correction, $F\left(\sigma_{v}\right)=a+b \times \sigma_{v}+c \times \sigma_{v}^{2}$, with their respective errors.

\begin{tabular}{|c|c|c|c|c|c|c|c|}
\hline \multicolumn{8}{|c|}{ Fitting coefficients and errors } \\
\hline Index & $A$ & $B$ & $\mathrm{rms}$ & $a$ & $b$ & $c$ & $\mathrm{rms}$ \\
\hline$E W$ & $(\AA)$ & & $(\AA)$ & & $\left(10^{-4} \mathrm{~km}^{-1} \mathrm{~s}\right)$ & $\left(10^{-6} \mathrm{~km}^{-2} \mathrm{~s}^{2}\right)$ & \\
\hline $\mathrm{Fe} 4383$ & 0.6920 & 0.94864 & 0.2720 & 0.9990 & 0.0699 & 2.2919 & 0.0149 \\
\hline $\mathrm{Ca} 4455$ & 1.3488 & 0.62615 & 0.2025 & 1.0085 & -5.0818 & 8.5130 & 0.1418 \\
\hline $\mathrm{Fe} 4531$ & -0.3046 & 1.22950 & 0.3710 & 1.0083 & -4.6272 & 1.6660 & 0.0574 \\
\hline $\mathrm{Fe} 4668$ & 0.1884 & 1.10060 & 0.3419 & 1.0005 & -0.6561 & 1.3835 & 0.0159 \\
\hline $\mathrm{H} \beta$ & -0.3155 & 1.12340 & 0.2026 & 1.0045 & -5.3309 & 1.7006 & 0.0754 \\
\hline $\mathrm{Fe} 5015$ & -0.5575 & 1.18350 & 0.6288 & 0.9913 & 3.5982 & 1.7919 & 0.0286 \\
\hline $\mathrm{Mg} \mathrm{b}$ & -0.2062 & 1.10380 & 0.2202 & 1.0019 & -1.3020 & 2.6367 & 0.0261 \\
\hline $\mathrm{Fe} 5270$ & -0.7634 & 1.27300 & 0.1708 & 0.9959 & 1.9521 & 1.7809 & 0.0215 \\
\hline $\mathrm{Fe} 5335$ & -0.3890 & 1.19720 & 0.1422 & 1.0005 & -1.5167 & 6.1129 & 0.0186 \\
\hline $\mathrm{Fe} 5406$ & -0.2449 & 1.22160 & 0.0725 & 1.0030 & -1.8058 & 5.2013 & 0.0298 \\
\hline Fe5709 & 0.0660 & 1.16050 & 0.2494 & 1.0041 & -2.5330 & 3.0240 & 0.0370 \\
\hline $\mathrm{Fe} 5782$ & 0.0465 & 1.23470 & 0.2014 & 0.9933 & 3.0617 & 4.7034 & 0.0420 \\
\hline $\mathrm{NaD}$ & 0.0183 & 0.97408 & 0.0962 & 1.0012 & -0.8740 & 1.3064 & 0.0116 \\
\hline$M A G$ & (mag) & & (mag) & & & & \\
\hline $\mathrm{Mg}_{1}$ & 0.0305 & 0.98239 & 0.0057 & 0.9989 & 0.6306 & 0.3009 & 0.0258 \\
\hline $\mathrm{Mg}_{2}$ & 0.0153 & 1.09010 & 0.0062 & 0.9995 & 0.1950 & 0.1189 & 0.0074 \\
\hline
\end{tabular}

\subsection{Influence of the sky subtraction and flux calibration}

The subtraction of the sky background was satisfactory for all galaxy and stellar spectra. However, the poor subtraction of the telluric emission line $\mathrm{HgI} \lambda 5460.74 \AA$ only affected the Fe5406 index in the outer regions of the galaxies. The residual of this line subtraction stays in the Fe5406 red continuum in the aperture spectra of NGC 1052 for $r \geq 11.78$ arcsec (major axis) or for all apertures (minor axis). The regions of all sky line residuals have been changed in the minor axis spectra of NGC 1052 by linear interpolations. For NGC 7796, the red continuum and bandpass of Fe5406 are affected by that sky line beyond the radial distance of 7.10 arcsec (major axis) or 6.93 arcsec (minor axis). The other sky line residuals do not affect the Lick index measurements because they do not occur in the index bandpasses as well as their continuum windows.

All Lick indices were also measured in the flux noncalibrated spectra (fnc) of the galaxies according to the original procedure of the Lick/IDS System. We have computed the index differences, $\triangle \mathrm{INDEX}=\mathrm{INDEX}_{\mathrm{fc}}-\mathrm{INDEX}_{\mathrm{fnc}}$, where fc denotes flux calibrated spectra. There is no systematic disagreement for all indices because these differences are comparable to or smaller than their errors: $\overline{\Delta E W} \approx+0.02 \AA$ and $\overline{\triangle M A G} \approx-0.009 \mathrm{mag}$. As an example, for the central major axis spectrum of NGC 1052 whose $S / N$ ratio is $83 \AA^{-1}$, these differences are: $\Delta E W(\AA)=+0.066$ for Fe4383, +0.010 for $\mathrm{Ca} 4455,+0.024$ for $\mathrm{Fe} 4531,+0.015$ for Fe4668, -0.009 for $\mathrm{H} \beta,-0.184$ for $\mathrm{Fe} 5015,+0.006$ for $\mathrm{Mg} \mathrm{b},+0.003$ for $\mathrm{Fe} 5270$, -0.005 for Fe5335, -0.003 for Fe5406, -0.003 for Fe5709, -0.005 for $\mathrm{Fe} 5782$ and -0.010 for $\mathrm{NaD}$; and $\triangle M A G$ (mag) $=$ -0.0103 for $\mathrm{Mg}_{1}$, and -0.0102 for $\mathrm{Mg}_{2}$. For the major axis spectrum of NGC 1052 at $r=20.21$ arcsec, whose $S / N$ ratio is $36 \AA^{-1}$, they are: $\Delta E W(\AA)=+0.102$ for Fe $4383,+0.015$ for $\mathrm{Ca} 4455,-0.003$ for $\mathrm{Fe} 4531,-0.041$ for $\mathrm{Fe} 4668,-0.007$ for $\mathrm{H} \beta$, -0.063 for Fe 5015, -0.004 for $\mathrm{Mg} \mathrm{b},+0.003$ for Fe5270, -0.008 for $\mathrm{Fe} 5335,-0.003$ for $\mathrm{Fe} 5406,-0.003$ for $\mathrm{Fe} 5709,-0.002$ for Fe5782 and -0.010 for $\mathrm{NaD}$; and $\triangle \mathrm{MAG}(\mathrm{mag})=-0.0118$ for $\mathrm{Mg}_{1}$, and -0.0115 for $\mathrm{Mg}_{2}$. Therefore, if there is some influence of the flux normalization precision over the Lick index measurements, it is negligible.

\subsection{Emission line corrections of some indices for NGC 1052}

For the aperture spectra of NGC 1052 up to the radial distance $r=3.56 \operatorname{arcsec}$ (major axis) and up to $r=4.92 \operatorname{arcsec}$ (minor axis), some Lick indices are affected by nebular emission lines of this LINER. The $\mathrm{H} \beta$ index, of course, is directly contaminated by its respective gas emission. The $\mathrm{Mg}_{1}, \mathrm{Mg}_{2}$ and Fe5015 features are severely affected by the [O III] $] 4958 \AA$ emission line present in their blue continuum windows. The bandpass of $\mathrm{Mg}_{2}$ is still contaminated by the [N I] $25198-5200 \AA$ emission doublet, hereafter [N I] $\lambda 5199 \AA$. Additionally, Fe5015 is strongly disturbed by the [O III] $\lambda 5007 \AA$ in its red continuum as well. The Mgb index has less contamination by the [NI] $\lambda 5199 \AA$ in its red continuum window. These indices must be carefully corrected, specifically the $\mathrm{Mg} b$ whose respective correction is more reliable. The corrections of $\mathrm{Mg} \mathrm{b}, \mathrm{Mg}_{1}$ and $\mathrm{Mg}_{2}$ were computed following the simple and accurate procedure described by Goudfrooij \& Emsellem (1996). The H $\beta$ correction should be only applied through suitable composite absorption-line templates that were constructed using the stellar population synthesis approach (Sect. 6). However, it is redundant because the synthesis method directly supplies the information that would be obtained from this stellar age indicator Lick index. On the other hand, the composite stellar population templates of the synthesis technique had to be adopted for computing the emission line corrections of the magnesium indices. These corrections are based on the equivalent widths of the nebular lines that must be measured in the pure emission spectra at the Lick/IDS resolution, i.e. after the subtraction of an absorption line template from the observed spectrum. The corrections due to the presence of an emission line inside an index continuum window or an index bandpass are computed as a function of the emission line equivalent width $E W_{\text {em.line }}$ measured in the continuum window or the index bandpass respectively, where $a$ (INDEX) and $b$ (INDEX) 
Table 7. The errors of the Lick indices for two spectra with different $S / N$ ratios: the Poisson error plus the $c z_{\text {helio }}$ uncertainty and the Lick System calibration errors.

\begin{tabular}{|c|c|c|c|}
\hline \multicolumn{4}{|c|}{ Lick index errors } \\
\hline Index & Poisson $+c z$ & linear corr. & $\sigma_{v}$ corr. \\
\hline$S / N=83 \AA^{-1}$ & & & \\
\hline$E W$ & $(\AA)$ & $(\AA)$ & $(\AA)$ \\
\hline $\mathrm{Fe} 4383$ & 0.128 & 0.170 & 0.173 \\
\hline Ca4455 & 0.065 & 0.088 & 0.385 \\
\hline $\mathrm{Fe} 4531$ & 0.091 & 0.292 & 0.165 \\
\hline Fe4668 & 0.137 & 0.258 & 0.178 \\
\hline $\mathrm{H} \beta$ & 0.060 & 0.151 & 0.187 \\
\hline Fe5015 & 0.114 & 0.466 & 0.047 \\
\hline $\mathrm{Mg} \mathrm{b}$ & 0.049 & 0.159 & 0.208 \\
\hline Fe5270 & 0.054 & 0.145 & 0.088 \\
\hline Fe5335 & 0.065 & 0.122 & 0.173 \\
\hline $\mathrm{Fe} 5406$ & 0.048 & 0.070 & 0.125 \\
\hline Fe5709 & 0.037 & 0.180 & 0.067 \\
\hline Fe5782 & 0.036 & 0.153 & 0.115 \\
\hline $\mathrm{NaD}$ & 0.038 & 0.063 & 0.095 \\
\hline$M A G$ & (mag) & (mag) & (mag) \\
\hline $\mathrm{Mg}_{1}$ & 0.0011 & 0.0038 & 0.0047 \\
\hline $\mathrm{Mg}_{2}$ & 0.0013 & 0.0044 & 0.0024 \\
\hline$S / N=26 \AA^{-1}$ & & & \\
\hline$E W$ & $(\AA)$ & $(\AA)$ & $(\AA)$ \\
\hline $\mathrm{Fe} 4383$ & 0.424 & 0.140 & 0.340 \\
\hline Ca4455 & 0.196 & 0.010 & 0.728 \\
\hline $\mathrm{Fe} 4531$ & 0.266 & 0.372 & 0.302 \\
\hline $\mathrm{Fe} 4668$ & 0.409 & 0.313 & 0.314 \\
\hline $\mathrm{H} \beta$ & 0.155 & 0.175 & 0.068 \\
\hline Fe5015 & 0.306 & 0.537 & 0.512 \\
\hline $\mathrm{Mg} \mathrm{b}$ & 0.157 & 0.181 & 0.320 \\
\hline Fe5270 & 0.158 & 0.201 & 0.230 \\
\hline Fe5335 & 0.194 & 0.172 & 0.535 \\
\hline Fe5406 & 0.147 & 0.114 & 0.245 \\
\hline Fe5709 & 0.120 & 0.207 & 0.092 \\
\hline Fe5782 & 0.112 & 0.188 & 0.219 \\
\hline $\mathrm{NaD}$ & 0.134 & 0.058 & 0.132 \\
\hline$M A G$ & (mag) & (mag) & (mag) \\
\hline $\mathrm{Mg}_{1}$ & 0.0034 & 0.0037 & 0.0035 \\
\hline $\mathrm{Mg}_{2}$ & 0.0039 & 0.0049 & 0.0025 \\
\hline
\end{tabular}

are constants of each index. For $\mathrm{Mg} \mathrm{b}, a \approx 1.127$ and for $\mathrm{Mg}_{1}$, $a \approx 0.4475$. For $\mathrm{Mg}_{2}, a \approx 0.4158$ and $b \approx-1.086 \mathrm{mag}$.

$\Delta E W=a(\operatorname{INDEX}) \times E W_{\mathrm{em} . l i n e}$,

$\triangle M A G=b(\mathrm{INDEX}) \times \frac{E W_{\mathrm{em} . l i n e}}{\Delta \lambda_{i}-E W_{\mathrm{INDEX}}}$.

The $\mathrm{Mg}_{1}$ and $\mathrm{Mg}_{2}$ corrections due to the [O III] $\lambda 4958 \AA$ line (in their blue continuums) were computed in the $E W$ scale and were transformed to the $M A G$ one. Table 8 presents the equivalent widths of the [O III] $\lambda 4958 \AA$ and [N I] $25199 \AA$ lines, measured in the index continuum windows (at $\lambda \lambda 4895.125-4957.625 \AA$, the blue one of $\mathrm{Mg}_{1}$ and $\mathrm{Mg}_{2}$, and at $\lambda \lambda 5191.375-5206.375 \AA$, the red one of $\mathrm{Mg} \mathrm{b}$ ) and the [N I] $\lambda 5199 \AA$ line in the $\mathrm{Mg}_{2}$ bandpass $(\lambda \lambda 5154.125-5196.625 \AA)$ as well as for the inner regions of NGC 1052. The equivalent widths of the emission lines were measured adopting a single Gaussian profile fit.

After taking into account the emission line corrections for the measurements of $\mathrm{Mg}_{1}, \mathrm{Mg}_{2}$ (2 corrections) and $\mathrm{Mg} \mathrm{b}$, these indices were calibrated to the Lick scale. Beyond those radial distances for $\mathrm{NGC} 1052, \mathrm{H} \beta, \mathrm{Mg} \mathrm{b}, \mathrm{Mg}_{1}$ and $\mathrm{Mg}_{2}$ were directly measured without corrections. The Fe5015 index was not corrected or adopted for both galaxies.

For NGC 7796, emission lines were not detected in the aperture spectra or the residual spectra (Sect. 6).

\subsection{External comparisons of some Lick indices}

In order to compare our measurements of the nuclear Lick indices, they must be corrected for the aperture effect as was the central velocity dispersion (see Sect. 2.2 and Jorgensen 1997). The adopted logarithmic radial gradients of the indices $\left(\nabla \mathrm{INDEX}=\frac{\Delta \log E W}{\Delta \log r}\right.$ or $\left.\frac{\Delta M A G}{\Delta \log r}\right)$ were $\nabla(\mathrm{Fe}$ index $)=-0.050$ including $\langle\mathrm{Fe}\rangle$ except $\nabla(\mathrm{Fe} 4668)=-0.080, \nabla(\mathrm{Ca} 4455)=$ $0, \nabla(\mathrm{NaD})=-0.090$, and $\nabla\left(\mathrm{Mg}_{1}\right)=\nabla\left(\mathrm{Mg}_{2}\right)=$ -0.038 mag dex $^{-1}$.

The central Lick indices of both galaxies from the current work are shown in Table 17, in which the mean literature values of some indices are also presented from several sources: Terlevich et al. (1981), Davies et al. (1987), Burstein et al. (1988), Couture \& Hardy (1988), Faber et al. (1989), Worthey et al. (1992), Carollo et al. (1993), Huchra et al. (1996) (all for $\mathrm{Mg}_{2}^{0}$ only), Trager et al. (1998) (not for $\mathrm{Mg}_{2}$ ), Beuing et al. (2002) and Thomas et al. (2005) (only for $\mathrm{Mgb},\langle\mathrm{Fe}\rangle$ and $\mathrm{H} \beta$ ) for NGC 1052 and Bertin et al. (1994), Golev \& Prugniel (1998) (both for $\mathrm{Mg}_{2}^{0}$ only), Beuing et al. (2002) and Thomas et al. (2005) (only for $\mathrm{Mg} \mathrm{b},\langle\mathrm{Fe}\rangle$ and $\mathrm{H} \beta$ ) for NGC 7796.

The estimate of the nuclear $\mathrm{Mg}_{2}$ based on the $\mathrm{Mg}_{2}^{0}-\sigma_{v}^{0}$ relation was made with the results of Bender et al. (1993). The predictions, using $\sigma_{v}^{0}\left(\mathrm{NGC} \mathrm{1052)}=204 \mathrm{~km} \mathrm{~s}^{-1}\right.$ and $\sigma_{v}^{0}$ $\left(\right.$ NGC 7796) $=253 \mathrm{~km} \mathrm{~s}^{-1}$, are very near to our measurements: $\mathrm{Mg}_{2}^{0}=0.296 \mathrm{mag}$ and $\mathrm{Mg}_{2}^{0}=0.315 \mathrm{mag}$, respectively for NGC 1052 and NGC 7796. Note that the literature values of $\mathrm{Mg}_{2}^{0}$ of NGC 7796 are smaller than our value and the predicted one (see Tables 1 and 17).

For all indices, the agreements with the literature values are good considering both errors. Note that the published Lick indices are not fully corrected (for the aperture effect and emission lines).

\section{Radial gradients of the Lick indices}

The calibrated/corrected Lick indices (except Fe5015) of the aperture spectra along both axes are shown in Tables 9 to 12 for NGC 1052 and Tables 13 to 16 for NGC 7796.

The Lick indices for which we have measured radial gradients along both axes of two galaxies are Fe4383, Ca4455, Fe4531, Fe4668, Mg b, Fe5270, Fe5335, 〈Fe $\rangle,$ Fe5406, Fe5709, $\mathrm{Fe} 5782$ and $\mathrm{NaD}$ (including $\mathrm{H} \beta, \mathrm{Mg}_{1}$ and $\mathrm{Mg}_{2}$ for NGC 7796). The $\langle\mathrm{Fe}\rangle$ index is an average value of two iron indices: $\langle\mathrm{Fe}\rangle=$ $(\mathrm{Fe} 5270+\mathrm{Fe} 5335) \div 2$.

For the major axis of NGC 1052, we have used the aperture spectra up to $r=20.2$ arcsec and for its minor axis up to 9.4 arcsec. For the major and minor axes of NGC 7796, we have used the spectra up to $r=13.2$ and 12.5 arcsec respectively (all data).

Some atomic line-strengths are contaminated by molecular absorption, such as $\mathrm{Fe} 4383$ that is influenced by $\mathrm{CH}$ lines and 
Table 8. Equivalent widths of [O III] $\lambda 4958 \AA$ line, measured in the blue continuum windows of $\mathrm{Mg}_{1}$ and $\mathrm{Mg}_{2}$ (that are the same), and [N I] $\lambda 5199 \AA$ line, measured either in the red continuum of $\mathrm{Mg} b$ and $\mathrm{Mg}_{2}$ bandpass, for the aperture spectra of the more central regions of NGC 1052 along both axes.

\begin{tabular}{l|ccc}
\hline \hline \multicolumn{5}{|c}{$E W(\AA)$} & \\
\hline$R(\operatorname{arcsec})$ & {$[\mathrm{O} \mathrm{III}] \lambda 4958_{\lambda \lambda 4895.125-4957.625}$} & {$[\mathrm{~N} \mathrm{I}] \lambda 5199_{\lambda \lambda 5191.375-5206.375}$} & {$[\mathrm{~N} \mathrm{I}] \lambda 5199_{\lambda \lambda 5154.125-5196.625}$} \\
\hline major axis & $-5.0 \pm 0.3$ & $-1.4 \pm 0.1$ & $-1.2 \pm 0.1$ \\
\hline 0.00 & $-5.5 \pm 0.5$ & $-1.0 \pm 0.1$ & $-0.9 \pm 0.1$ \\
\hline $1.10 \mathrm{SE}$ & $-4.1 \pm 0.6$ & $-0.8 \pm 0.1$ & $-0.7 \pm 0.1$ \\
$3.56 \mathrm{SE}$ & $-4.5 \pm 0.2$ & $-0.9 \pm 0.1$ & $-0.8 \pm 0.1$ \\
\hline $1.08 \mathrm{NW}$ & $-2.1 \pm 0.7$ & $-0.4 \pm 0.1$ & $-0.4 \pm 0.1$ \\
$3.54 \mathrm{NW}$ & & & - \\
\hline minor axis & $-4.1 \pm 0.2$ & $-0.70 \pm 0.05$ & - \\
\hline 0.00 & $-3.1 \pm 1.5$ & $-0.88 \pm 0.09$ & - \\
\hline $2.17 \mathrm{SW}$ & - & $-0.81 \pm 0.10$ & - \\
$4.90 \mathrm{SW}$ & $-3.2 \pm 0.6$ & $-0.82 \pm 0.05$ & $-3.1 \pm 0.2$ \\
\hline $2.19 \mathrm{NE}$ & - & $-0.15 \pm 0.10$ & \\
$4.92 \mathrm{NE}$ & & & \\
\hline
\end{tabular}

Table 9. Lick indices as a function of the distance to the galaxy center - NGC 1052, major axis.

\begin{tabular}{|c|c|c|c|c|c|c|c|c|}
\hline \multicolumn{9}{|c|}{$E W(\AA)$ indices } \\
\hline$R(\operatorname{arcsec})$ & Fe4383 & Ca4455 & $\mathrm{Fe} 4531$ & $\mathrm{Fe} 4668$ & $\mathrm{Mg} \mathrm{b}$ & Fe5270 & Fe5335 & Fe5406 \\
\hline 0.00 & $6.14 \pm 0.30$ & $2.35 \pm 0.30$ & $4.42 \pm 0.32$ & $8.81 \pm 0.35$ & $4.21 \pm 0.23$ & $2.46 \pm 0.17$ & $2.61 \pm 0.21$ & $2.02 \pm 0.15$ \\
\hline $1.10 \mathrm{SE}$ & $6.52 \pm 0.30$ & $2.50 \pm 0.30$ & $3.60 \pm 0.32$ & $8.53 \pm 0.36$ & $5.03 \pm 0.23$ & $2.29 \pm 0.17$ & $2.49 \pm 0.21$ & $1.96 \pm 0.14$ \\
\hline $3.56 \mathrm{SE}$ & $5.89 \pm 0.33$ & $2.44 \pm 0.32$ & $3.77 \pm 0.35$ & $8.80 \pm 0.40$ & $4.37 \pm 0.25$ & $2.55 \pm 0.19$ & $2.20 \pm 0.23$ & $1.82 \pm 0.16$ \\
\hline 6.84 SE & $5.72 \pm 0.40$ & $2.42 \pm 0.35$ & $3.07 \pm 0.39$ & $8.18 \pm 0.46$ & $5.09 \pm 0.27$ & $2.67 \pm 0.23$ & $2.05 \pm 0.28$ & $1.73 \pm 0.20$ \\
\hline $11.80 \mathrm{SE}$ & $6.11 \pm 0.44$ & $2.38 \pm 0.37$ & $3.50 \pm 0.42$ & $6.86 \pm 0.50$ & $4.75 \pm 0.29$ & $2.58 \pm 0.25$ & $2.74 \pm 0.33$ & $2.12 \pm 0.23$ \\
\hline $20.21 \mathrm{SE}$ & $4.62 \pm 0.47$ & $2.91 \pm 0.43$ & $4.42 \pm 0.47$ & $8.39 \pm 0.55$ & $4.10 \pm 0.30$ & $2.84 \pm 0.27$ & $2.49 \pm 0.35$ & $2.30 \pm 0.25$ \\
\hline $1.08 \mathrm{NW}$ & $5.98 \pm 0.30$ & $2.43 \pm 0.30$ & $3.78 \pm 0.32$ & $8.93 \pm 0.36$ & $4.94 \pm 0.28$ & $2.59 \pm 0.17$ & $2.67 \pm 0.21$ & $1.93 \pm 0.15$ \\
\hline $3.54 \mathrm{NW}$ & $6.79 \pm 0.33$ & $2.46 \pm 0.31$ & $3.70 \pm 0.35$ & $8.22 \pm 0.36$ & $5.07 \pm 0.24$ & $2.49 \pm 0.19$ & $2.71 \pm 0.23$ & $1.99 \pm 0.16$ \\
\hline $6.81 \mathrm{NW}$ & $6.44 \pm 0.40$ & $2.33 \pm 0.35$ & $3.61 \pm 0.39$ & $7.77 \pm 0.45$ & $5.16 \pm 0.26$ & $3.01 \pm 0.22$ & $2.62 \pm 0.28$ & $1.92 \pm 0.19$ \\
\hline $11.78 \mathrm{NW}$ & $4.86 \pm 0.42$ & $2.28 \pm 0.36$ & $2.85 \pm 0.40$ & $8.12 \pm 0.50$ & $4.54 \pm 0.28$ & $2.80 \pm 0.25$ & $2.48 \pm 0.31$ & $1.62 \pm 0.21$ \\
\hline $20.19 \mathrm{NW}$ & $5.62 \pm 0.45$ & $2.36 \pm 0.36$ & $2.83 \pm 0.43$ & $5.55 \pm 0.51$ & $4.55 \pm 0.28$ & $3.03 \pm 0.26$ & $1.76 \pm 0.30$ & $1.39 \pm 0.21$ \\
\hline
\end{tabular}

Table 10. Lick indices as a function of the distance to the galaxy center - NGC 1052, major axis.

\begin{tabular}{l|cccccc}
\hline \hline \multicolumn{7}{c}{$E W(\AA)$ and $M A G(\mathrm{mag})$ indices } \\
\hline$R(\operatorname{arcsec})$ & Fe5709 & Fe5782 & Na D & $\mathrm{H} \beta$ & $\mathrm{Mg}_{1}$ & $\mathrm{Mg}_{2}$ \\
\hline 0.00 & $1.09 \pm 0.16$ & $0.98 \pm 0.17$ & $6.46 \pm 0.11$ & - & $0.181 \pm 0.004$ & $0.331 \pm 0.004$ \\
\hline $1.10 \mathrm{SE}$ & $0.94 \pm 0.16$ & $0.91 \pm 0.17$ & $6.03 \pm 0.11$ & - & $0.172 \pm 0.004$ & $0.303 \pm 0.004$ \\
$3.56 \mathrm{SE}$ & $0.91 \pm 0.17$ & $0.86 \pm 0.18$ & $4.59 \pm 0.12$ & - & $0.148 \pm 0.004$ & $0.304 \pm 0.004$ \\
$6.84 \mathrm{SE}$ & $1.20 \pm 0.20$ & $0.39 \pm 0.20$ & $3.84 \pm 0.14$ & $0.68 \pm 0.19$ & $0.174 \pm 0.005$ & $0.329 \pm 0.005$ \\
$11.80 \mathrm{SE}$ & $1.31 \pm 0.22$ & $0.58 \pm 0.22$ & $3.33 \pm 0.15$ & $1.28 \pm 0.22$ & $0.138 \pm 0.006$ & $0.298 \pm 0.006$ \\
20.21 SE & $1.22 \pm 0.23$ & $0.26 \pm 0.22$ & $3.36 \pm 0.16$ & $1.08 \pm 0.23$ & $0.148 \pm 0.006$ & $0.294 \pm 0.006$ \\
\hline $1.08 \mathrm{NW}$ & $1.11 \pm 0.16$ & $0.91 \pm 0.17$ & $6.31 \pm 0.11$ & - & $0.178 \pm 0.004$ & $0.322 \pm 0.004$ \\
$3.54 \mathrm{NW}$ & $1.02 \pm 0.17$ & $0.73 \pm 0.18$ & $5.41 \pm 0.12$ & - & $0.164 \pm 0.004$ & $0.317 \pm 0.004$ \\
$6.81 \mathrm{NW}$ & $1.23 \pm 0.20$ & $1.05 \pm 0.21$ & $4.51 \pm 0.14$ & $0.61 \pm 0.18$ & $0.168 \pm 0.005$ & $0.330 \pm 0.005$ \\
$11.78 \mathrm{NW}$ & $0.94 \pm 0.20$ & $0.62 \pm 0.22$ & $3.91 \pm 0.15$ & $1.19 \pm 0.21$ & $0.147 \pm 0.006$ & $0.304 \pm 0.006$ \\
20.19 NW & $1.06 \pm 0.21$ & $0.74 \pm 0.22$ & $3.32 \pm 0.16$ & $1.28 \pm 0.23$ & $0.125 \pm 0.006$ & $0.291 \pm 0.006$ \\
\hline
\end{tabular}

Table notes. Columns 6 and 7: mag unity.

Table 11. Lick indices as a function of the distance to the galaxy center - NGC 1052, minor axis.

\begin{tabular}{l|cccccccc}
\hline \hline \multicolumn{7}{c}{$E W(\AA)$ indices } \\
\hline$R(\operatorname{arcsec})$ & Fe4383 & Ca4455 & Fe4531 & Fe4668 & Mg b & Fe5270 & Fe5335 & Fe5406 \\
\hline 0.00 & $7.09 \pm 0.63$ & $2.39 \pm 0.48$ & $3.84 \pm 0.54$ & $8.46 \pm 0.66$ & $5.44 \pm 0.39$ & $2.60 \pm 0.30$ & $2.86 \pm 0.43$ & $2.09 \pm 0.29$ \\
\hline $2.17 \mathrm{SW}$ & $5.80 \pm 0.84$ & $2.39 \pm 0.48$ & $3.67 \pm 0.69$ & $8.84 \pm 0.86$ & $4.92 \pm 0.47$ & $2.59 \pm 0.38$ & $2.48 \pm 0.54$ & $1.98 \pm 0.37$ \\
$4.90 \mathrm{SW}$ & $4.99 \pm 1.17$ & $2.67 \pm 0.71$ & $2.17 \pm 0.90$ & $6.64 \pm 1.16$ & $4.07 \pm 0.55$ & $1.88 \pm 0.49$ & $2.49 \pm 0.67$ & $1.95 \pm 0.47$ \\
$9.41 \mathrm{SW}$ & $6.68 \pm 1.66$ & $2.42 \pm 0.95$ & $3.61 \pm 1.25$ & $7.21 \pm 1.60$ & $5.51 \pm 0.76$ & $3.40 \pm 0.70$ & $3.03 \pm 0.98$ & $2.33 \pm 0.70$ \\
\hline $2.19 \mathrm{NE}$ & $6.95 \pm 0.86$ & $2.56 \pm 0.61$ & $3.27 \pm 0.69$ & $8.68 \pm 0.87$ & $5.03 \pm 0.48$ & $2.61 \pm 0.39$ & $3.24 \pm 0.58$ & $1.99 \pm 0.38$ \\
$4.92 \mathrm{NE}$ & $5.32 \pm 1.25$ & $2.40 \pm 0.74$ & $3.43 \pm 0.97$ & $7.56 \pm 1.23$ & $5.53 \pm 0.60$ & $2.59 \pm 0.53$ & $2.66 \pm 0.74$ & $2.01 \pm 0.52$ \\
$9.43 \mathrm{NE}$ & $4.27 \pm 1.75$ & $1.90 \pm 0.69$ & $1.80 \pm 1.16$ & $4.12 \pm 1.51$ & $4.38 \pm 0.63$ & $1.39 \pm 0.63$ & $1.56 \pm 0.77$ & $1.49 \pm 0.56$ \\
\hline
\end{tabular}


Table 12. Lick indices as a function of the distance to the galaxy center - NGC 1052, minor axis.

\begin{tabular}{l|cccccc}
\hline \hline \multicolumn{7}{c}{$E W(\AA)$ and $M A G(\mathrm{mag})$ indices } \\
\hline$R$ (arcsec) & Fe5709 & Fe5782 & Na D & $\mathrm{H} \beta$ & $\mathrm{Mg}_{1}$ & $\mathrm{Mg}_{2}$ \\
\hline 0.00 & $1.08 \pm 0.25$ & $0.87 \pm 0.27$ & $6.74 \pm 0.21$ & - & $0.183 \pm 0.007$ & $0.285 \pm 0.007$ \\
\hline $2.17 \mathrm{SW}$ & $0.91 \pm 0.29$ & $1.15 \pm 0.34$ & $5.85 \pm 0.25$ & - & $0.172 \pm 0.008$ & $0.306 \pm 0.008$ \\
4.90 SW & $0.95 \pm 0.36$ & $1.21 \pm 0.42$ & $5.41 \pm 0.31$ & - & $0.178 \pm 0.010$ & $0.348 \pm 0.011$ \\
9.41 SW & $0.90 \pm 0.47$ & $0.76 \pm 0.55$ & $2.04 \pm 0.40$ & $0.88 \pm 0.56$ & $0.168 \pm 0.012$ & $0.333 \pm 0.014$ \\
\hline $2.19 \mathrm{NE}$ & $0.95 \pm 0.30$ & $1.26 \pm 0.35$ & $5.52 \pm 0.25$ & - & $0.179 \pm 0.008$ & $0.303 \pm 0.009$ \\
$4.92 \mathrm{NE}$ & $1.25 \pm 0.39$ & $1.42 \pm 0.46$ & $3.93 \pm 0.32$ & - & $0.183 \pm 0.011$ & $0.460 \pm 0.012$ \\
$9.43 \mathrm{NE}$ & $1.18 \pm 0.45$ & $1.30 \pm 0.50$ & $2.90 \pm 0.38$ & $0.73 \pm 0.54$ & $0.157 \pm 0.012$ & $0.301 \pm 0.014$ \\
\hline
\end{tabular}

Table notes. Columns 6 and 7: mag unity.

Table 13. Lick indices as a function of the distance to the galaxy center - NGC 7796, major axis.

\begin{tabular}{|c|c|c|c|c|c|c|c|c|}
\hline \multicolumn{9}{|c|}{$E W(\AA)$ indices } \\
\hline$R(\operatorname{arcsec})$ & $\mathrm{Fe} 4383$ & Ca4455 & $\mathrm{Fe} 4531$ & $\mathrm{Fe} 4668$ & $\mathrm{Mg} \mathrm{b}$ & Fe5270 & Fe5335 & Fe5406 \\
\hline 0.00 & $5.25 \pm 0.35$ & $2.65 \pm 0.36$ & $3.41 \pm 0.35$ & $9.22 \pm 0.41$ & $4.93 \pm 0.25$ & $2.84 \pm 0.20$ & $2.42 \pm 0.26$ & $1.83 \pm 0.18$ \\
\hline $1.09 \mathrm{~S}$ & $5.28 \pm 0.38$ & $2.82 \pm 0.41$ & $3.28 \pm 0.36$ & $8.37 \pm 0.44$ & $5.16 \pm 0.27$ & $2.68 \pm 0.22$ & $2.28 \pm 0.30$ & $1.60 \pm 0.19$ \\
\hline $3.55 \mathrm{~S}$ & $5.22 \pm 0.48$ & $2.71 \pm 0.47$ & $3.59 \pm 0.44$ & $7.50 \pm 0.53$ & $5.47 \pm 0.34$ & $2.80 \pm 0.27$ & $2.87 \pm 0.39$ & $1.30 \pm 0.24$ \\
\hline $7.10 \mathrm{~S}$ & $5.05 \pm 0.58$ & $2.70 \pm 0.55$ & $4.11 \pm 0.53$ & $7.38 \pm 0.65$ & $5.45 \pm 0.41$ & $3.35 \pm 0.33$ & $3.14 \pm 0.51$ & $1.14 \pm 0.29$ \\
\hline $13.21 \mathrm{~S}$ & $5.26 \pm 0.68$ & $2.72 \pm 0.59$ & $3.59 \pm 0.59$ & $5.82 \pm 0.73$ & $5.42 \pm 0.44$ & $3.01 \pm 0.37$ & $2.25 \pm 0.52$ & $0.51 \pm 0.30$ \\
\hline $1.09 \mathrm{~N}$ & $5.27 \pm 0.36$ & $2.50 \pm 0.35$ & $3.47 \pm 0.36$ & $9.12 \pm 0.43$ & $5.06 \pm 0.26$ & $3.12 \pm 0.21$ & $2.65 \pm 0.28$ & $1.84 \pm 0.18$ \\
\hline $3.55 \mathrm{~N}$ & $4.60 \pm 0.45$ & $2.63 \pm 0.44$ & $3.76 \pm 0.43$ & $8.42 \pm 0.52$ & $4.99 \pm 0.32$ & $3.10 \pm 0.26$ & $2.56 \pm 0.36$ & $1.81 \pm 0.24$ \\
\hline $7.10 \mathrm{~N}$ & $4.68 \pm 0.53$ & $2.23 \pm 0.45$ & $3.92 \pm 0.51$ & $7.95 \pm 0.61$ & $4.71 \pm 0.35$ & $3.06 \pm 0.30$ & $3.42 \pm 0.45$ & $1.45 \pm 0.27$ \\
\hline $13.21 \mathrm{~N}$ & $4.49 \pm 0.66$ & $2.53 \pm 0.55$ & $4.70 \pm 0.60$ & $7.36 \pm 0.72$ & $4.35 \pm 0.41$ & $3.60 \pm 0.37$ & $3.26 \pm 0.55$ & $1.35 \pm 0.32$ \\
\hline
\end{tabular}

Table 14. Lick indices as a function of the distance to the galaxy center - NGC 7796, major axis.

\begin{tabular}{l|cccccc}
\hline \hline \multicolumn{7}{c}{$E W(\AA)$ and $M A G(\mathrm{mag})$ indices } \\
\hline$R(\operatorname{arcsec})$ & Fe5709 & Fe5782 & Na D & $\mathrm{H} \beta$ & $\mathrm{Mg}_{1}$ & $\mathrm{Mg}_{2}$ \\
\hline 0.00 & $0.81 \pm 0.18$ & $0.66 \pm 0.19$ & $5.06 \pm 0.13$ & $1.56 \pm 0.19$ & $0.155 \pm 0.005$ & $0.345 \pm 0.005$ \\
\hline $1.09 \mathrm{~S}$ & $0.98 \pm 0.19$ & $0.51 \pm 0.20$ & $5.11 \pm 0.14$ & $1.46 \pm 0.19$ & $0.157 \pm 0.005$ & $0.338 \pm 0.005$ \\
$3.55 \mathrm{~S}$ & $1.09 \pm 0.23$ & $0.58 \pm 0.25$ & $4.82 \pm 0.17$ & $1.79 \pm 0.24$ & $0.191 \pm 0.005$ & $0.359 \pm 0.006$ \\
$7.10 \mathrm{~S}$ & $0.88 \pm 0.26$ & $0.91 \pm 0.30$ & $4.47 \pm 0.21$ & $1.41 \pm 0.27$ & $0.147 \pm 0.006$ & $0.334 \pm 0.007$ \\
$13.21 \mathrm{~S}$ & $0.05 \pm 0.26$ & $-0.18 \pm 0.28$ & $4.26 \pm 0.23$ & $1.43 \pm 0.30$ & $0.153 \pm 0.007$ & $0.311 \pm 0.008$ \\
\hline $1.09 \mathrm{~N}$ & $0.94 \pm 0.19$ & $0.68 \pm 0.20$ & $4.99 \pm 0.13$ & $1.54 \pm 0.19$ & $0.173 \pm 0.005$ & $0.356 \pm 0.005$ \\
$3.55 \mathrm{~N}$ & $0.87 \pm 0.22$ & $0.67 \pm 0.24$ & $4.39 \pm 0.16$ & $1.66 \pm 0.23$ & $0.184 \pm 0.006$ & $0.353 \pm 0.006$ \\
$7.10 \mathrm{~N}$ & $0.80 \pm 0.24$ & $0.83 \pm 0.28$ & $4.48 \pm 0.19$ & $1.52 \pm 0.25$ & $0.150 \pm 0.006$ & $0.319 \pm 0.007$ \\
$13.21 \mathrm{~N}$ & $0.55 \pm 0.27$ & $0.77 \pm 0.32$ & $3.99 \pm 0.23$ & $1.02 \pm 0.28$ & $0.140 \pm 0.007$ & $0.306 \pm 0.008$ \\
\hline
\end{tabular}

Table notes. Columns 6 and 7: mag unity.

Fe4668 by $C_{2}$ lines of the Swan System (Tripicco \& Bell 1995). Thomas et al. (2003a) re-classified Fe4668 as $\mathrm{C}_{2} 4668$. However they affirmed that Fe4383 index is still very sensitive to the iron abundance.

The blue continuum of the $\mathrm{NaD}$ index is partially contaminated by the same sodium absorption of the interestellar medium of the Galaxy, but this is equal for all spectra.

The distances to the galaxy center are normalized by the effective radius $r_{\mathrm{e}}$ that is corrected considering the apparent ellipticity $\epsilon$, so that $r_{\mathrm{e}}^{\text {corr }}=r_{\mathrm{e}}(1-\epsilon)^{-1 / 2}$ in the major axis direction or $r_{\mathrm{e}}^{\text {corr }}=r_{\mathrm{e}}(1-\epsilon)^{1 / 2}$ in the minor one (Davies et al. 1993 and Kobayashi \& Arimoto 1999).

We have computed the radial gradients of the Lick indices as a function of the logarithm of the normalized radius through a linear regression fit,

$\operatorname{Index}(r)=\operatorname{Index}_{\mathrm{e}}+\frac{\Delta \operatorname{Index}}{\Delta \log r} \cdot \log \frac{r}{r_{\mathrm{e}}^{\text {corr }}}$,

where $\operatorname{Index}_{\mathrm{e}}=\operatorname{Index}\left(\log \frac{r}{r_{\mathrm{e}}^{\text {corr }}}=0\right)=\operatorname{Index}\left(r=r_{\mathrm{e}}^{\text {corr }}\right)$ is the linear regression constant. The linear fits are computed by the least square method that takes into account the data errors only on the Lick indices (like Kobayashi \& Arimoto 1999).

In Table 18, we have shown the respective results of the fits: the radial gradients and the index values at one $r_{\mathrm{e}}^{\text {corr }}$.

In Figs. 5-7, the Lick indices as a function of the logarithm of the normalized radius along both axes are shown with their respective linear fits for NGC 1052. Figure 5 shows the gradients of Fe4383, Fe4531, Fe4668, Fe5270, Fe5335 and $\langle\mathrm{Fe}\rangle$. Figure 6 shows the gradients of Fe5406, Fe5709, Fe5782, Ca4455, Mg b and $\mathrm{Na} \mathrm{D}$. Figure 7 presents the radial gradients of $\mathrm{Mg}_{1}$ and $\mathrm{Mg}_{2}$.

In Figs. 8-10, the Lick indices as a function of the logarithm of the normalized radius along both axes are presented with their respective linear fits for NGC 7796. Figure 8 shows the gradients of Fe4383, Fe4531, Fe4668, Fe5270, Fe5335 and $\langle\mathrm{Fe}\rangle$. Figure 9 shows the gradients of Fe5406, Fe5709, Fe5782, Ca4455, Mg b and $\mathrm{NaD}$. Figure 10 presents the radial gradients of $\mathrm{H} \beta, \mathrm{Mg}_{1}$ and $\mathrm{Mg}_{2}$.

For NGC 1052, the majority of the gradients of the iron indices are practically zero considering their errors: Fe4531, $\mathrm{Fe} 5335,\langle\mathrm{Fe}\rangle, \mathrm{Fe} 5406$ and Fe5709 along the major axis and 
Table 15. Lick indices as a function of the distance to the galaxy center - NGC 7796, minor axis.

\begin{tabular}{|c|c|c|c|c|c|c|c|c|}
\hline \multicolumn{9}{|c|}{$E W(\AA)$ indices } \\
\hline$R(\operatorname{arcsec})$ & Fe4383 & Ca4455 & Fe4531 & $\mathrm{Fe} 4668$ & $\mathrm{Mg} \mathrm{b}$ & Fe5270 & Fe5335 & Fe5406 \\
\hline 0.00 & $5.33 \pm 0.42$ & $2.88 \pm 0.44$ & $3.05 \pm 0.39$ & $8.33 \pm 0.48$ & $5.10 \pm 0.30$ & $2.50 \pm 0.24$ & $2.60 \pm 0.33$ & $1.67 \pm 0.22$ \\
\hline $1.09 \mathrm{E}$ & $5.93 \pm 0.45$ & $2.95 \pm 0.46$ & $3.11 \pm 0.41$ & $7.85 \pm 0.50$ & $4.91 \pm 0.31$ & $2.51 \pm 0.25$ & $2.57 \pm 0.36$ & $1.49 \pm 0.23$ \\
\hline $3.55 \mathrm{E}$ & $5.43 \pm 0.58$ & $2.64 \pm 0.53$ & $2.70 \pm 0.49$ & $6.72 \pm 0.62$ & $4.66 \pm 0.37$ & $2.88 \pm 0.32$ & $3.05 \pm 0.48$ & $1.31 \pm 0.29$ \\
\hline $6.93 \mathrm{E}$ & $4.19 \pm 0.79$ & $2.13 \pm 0.60$ & $2.70 \pm 0.64$ & $2.59 \pm 0.80$ & $4.66 \pm 0.49$ & $4.03 \pm 0.45$ & $2.32 \pm 0.61$ & $1.44 \pm 0.41$ \\
\hline $12.50 \mathrm{E}$ & $2.90 \pm 0.94$ & $2.40 \pm 0.72$ & $1.90 \pm 0.74$ & $8.03 \pm 1.02$ & $3.99 \pm 0.55$ & $3.54 \pm 0.51$ & $2.46 \pm 0.73$ & $0.58 \pm 0.43$ \\
\hline $1.09 \mathrm{~W}$ & $5.42 \pm 0.44$ & $2.74 \pm 0.44$ & $3.08 \pm 0.41$ & $7.96 \pm 0.50$ & $5.23 \pm 0.31$ & $2.48 \pm 0.25$ & $2.68 \pm 0.35$ & $1.84 \pm 0.23$ \\
\hline $3.55 \mathrm{~W}$ & $4.77 \pm 0.54$ & $2.94 \pm 0.54$ & $2.98 \pm 0.48$ & $6.41 \pm 0.60$ & $5.02 \pm 0.38$ & $2.69 \pm 0.31$ & $2.80 \pm 0.46$ & $1.54 \pm 0.29$ \\
\hline $6.93 \mathrm{~W}$ & $4.66 \pm 0.72$ & $2.56 \pm 0.56$ & $2.24 \pm 0.61$ & $3.83 \pm 0.79$ & $5.01 \pm 0.45$ & $2.09 \pm 0.39$ & $2.69 \pm 0.56$ & $1.42 \pm 0.36$ \\
\hline $12.50 \mathrm{~W}$ & $2.57 \pm 0.88$ & $1.45 \pm 0.51$ & $2.02 \pm 0.73$ & $-4.62 \pm 0.94$ & $4.33 \pm 0.49$ & $3.10 \pm 0.47$ & $2.92 \pm 0.64$ & $0.84 \pm 0.40$ \\
\hline
\end{tabular}

Table 16. Lick indices as a function of the distance to the galaxy center - NGC 7796, minor axis.

\begin{tabular}{l|cccccc}
\hline \hline \multicolumn{7}{c}{$E W(\AA)$ and $M A G(\mathrm{mag})$ indices } \\
\hline$R(\operatorname{arcsec})$ & Fe5709 & Fe5782 & $\mathrm{Na} \mathrm{D}$ & $\mathrm{H} \beta$ & $\mathrm{Mg}_{1}$ & $\mathrm{Mg}_{2}$ \\
\hline 0.00 & $0.68 \pm 0.20$ & $0.50 \pm 0.22$ & $4.74 \pm 0.15$ & $1.11 \pm 0.20$ & $0.178 \pm 0.005$ & $0.366 \pm 0.006$ \\
\hline $1.09 \mathrm{E}$ & $0.75 \pm 0.21$ & $0.54 \pm 0.23$ & $4.85 \pm 0.16$ & $1.19 \pm 0.21$ & $0.180 \pm 0.006$ & $0.360 \pm 0.006$ \\
$3.55 \mathrm{E}$ & $1.17 \pm 0.27$ & $0.31 \pm 0.28$ & $4.55 \pm 0.21$ & $1.08 \pm 0.25$ & $0.169 \pm 0.007$ & $0.326 \pm 0.007$ \\
$6.93 \mathrm{E}$ & $0.76 \pm 0.32$ & $0.83 \pm 0.38$ & $4.61 \pm 0.28$ & $2.27 \pm 0.36$ & $0.148 \pm 0.008$ & $0.331 \pm 0.009$ \\
$12.50 \mathrm{E}$ & $0.17 \pm 0.35$ & $-0.09 \pm 0.39$ & $4.53 \pm 0.32$ & $1.52 \pm 0.40$ & $0.158 \pm 0.009$ & $0.341 \pm 0.010$ \\
\hline $1.09 \mathrm{~W}$ & $0.90 \pm 0.16$ & $0.62 \pm 0.23$ & $4.46 \pm 0.08$ & $1.26 \pm 0.21$ & $0.172 \pm 0.006$ & $0.363 \pm 0.006$ \\
$3.55 \mathrm{~W}$ & $0.84 \pm 0.20$ & $0.70 \pm 0.28$ & $4.32 \pm 0.10$ & $1.31 \pm 0.25$ & $0.163 \pm 0.007$ & $0.345 \pm 0.007$ \\
$6.93 \mathrm{~W}$ & $0.08 \pm 0.28$ & $0.65 \pm 0.34$ & $3.95 \pm 0.25$ & $1.57 \pm 0.33$ & $0.159 \pm 0.008$ & $0.340 \pm 0.009$ \\
$12.50 \mathrm{~W}$ & $0.89 \pm 0.35$ & $-0.02 \pm 0.36$ & $4.43 \pm 0.29$ & $1.40 \pm 0.38$ & $0.148 \pm 0.009$ & $0.283 \pm 0.0010$ \\
\hline
\end{tabular}

Table notes. Columns 6 and 7: mag unity.

Table 17. Lick index external comparisons: galaxy central values of Lick indices (major axis only).

\begin{tabular}{l|cccc}
\hline \hline \multicolumn{5}{c}{$E W(\AA)$ and $M A G(\mathrm{mag})$ indices } \\
\hline Index & NGC 1052 & NGC 7796 & \\
\hline $\mathrm{Fe} 4383^{0}$ & $5.62 \pm 0.30$ & $6.11 \pm 0.45$ & $4.97 \pm 0.35$ & literature \\
$\mathrm{Ca} 4455^{0}$ & $2.36 \pm 0.30$ & $2.10 \pm 0.21$ & $2.66 \pm 0.36$ & - \\
$\mathrm{Fe} 4531^{0}$ & $3.36 \pm 0.47$ & $3.62 \pm 0.30$ & $3.22 \pm 0.35$ & - \\
$\mathrm{Fe} 4668^{0}$ & $7.65 \pm 0.35$ & $8.50 \pm 0.44$ & $8.45 \pm 0.41$ & - \\
$\mathrm{Mg} \mathrm{b}$ & $3.83 \pm 0.23$ & $5.63 \pm 0.14$ & $4.67 \pm 0.25$ & $5.20 \pm 0.25$ \\
$\mathrm{Fe} 5270^{0}$ & $2.25 \pm 0.17$ & $2.81 \pm 0.04$ & $2.69 \pm 0.20$ & $3.63 \pm 0.05$ \\
$\mathrm{Fe} 5335^{0}$ & $2.39 \pm 0.21$ & $2.74 \pm 0.07$ & $2.30 \pm 0.26$ & $2.98 \pm 0.06$ \\
$\mathrm{Fe} 5406^{0}$ & $1.85 \pm 0.15$ & $1.82 \pm 0.02$ & $1.73 \pm 0.18$ & $1.87 \pm 0.05$ \\
$\mathrm{Fe} 5709^{0}$ & $1.00 \pm 0.16$ & $0.98 \pm 0.02$ & $0.76 \pm 0.18$ & $1.01 \pm 0.04$ \\
$\mathrm{Fe} 5782^{0}$ & $0.90 \pm 0.17$ & $0.93 \pm 0.02$ & $0.62 \pm 0.19$ & $1.02 \pm 0.04$ \\
$\mathrm{Na} \mathrm{D}$ & $5.52 \pm 0.11$ & $6.10 \pm 0.03$ & $4.58 \pm 0.13$ & $5.48 \pm 0.06$ \\
$\langle\mathrm{Fe}\rangle^{0}$ & $2.32 \pm 0.21$ & $2.78 \pm 0.02$ & $2.49 \pm 0.33$ & $3.04 \pm 0.37$ \\
$\mathrm{H} \beta^{0}$ & - & $1.22 \pm ?$ & $1.57 \pm 0.19$ & $1.64 \pm 0.16$ \\
$\mathrm{Mg}_{1}^{0}$ & $0.149 \pm 0.004$ & $0.193 \pm 0.001$ & $0.137 \pm 0.005$ & $0.165 \pm 0.002$ \\
$\mathrm{Mg}_{2}^{0}$ & $0.293 \pm 0.004$ & $0.303 \pm 0.017$ & $0.326 \pm 0.005$ & $0.267 \pm 0.051$ \\
\hline
\end{tabular}

Fe5270, Fe5406, Fe5709 and Fe5782 along the minor axis. One of them is positive: that of Fe5270 in the major axis. The $\mathrm{Mg} \mathrm{b}$, $\mathrm{Mg}_{1}$ and $\mathrm{Mg}_{2}$ gradients are negative. The $\mathrm{Mg}_{2}$ minor axis gradient was seriously affected by the emission line corrections for the aperture spectrum at $r=4.92 \operatorname{arcsec}$ (see Tables 12 and 18). However, the $\mathrm{Mg}_{1}$ and $\mathrm{Mg}_{2}$ gradients are weaker than the average ones for ellipticals $\left(-0.038 \mathrm{mag} \mathrm{dex}^{-1}\right.$ as denoted by Jorgensen 1997) and the gradients of other Fe indices are still negative. The Ca4455 gradient is also zero along both axes. The $\mathrm{Na} \mathrm{D}$ gradient is strongly negative in both axes and it is greater than the $\mathrm{Mgb}$ one. The $\mathrm{NaD}$ is probably contaminated by the interstellar absorption of NGC 1052 which should increase inwards. If $\mathrm{Fe} 4383$ and $\mathrm{Fe} 4668$ are actually contaminated by $\mathrm{CH}$ and $\mathrm{C}_{2}$ lines, there might be a negative radial gradient of the carbon stellar abundance in NGC 1052.

For NGC 7796, the gradients of the Lick indices along the major axis are equal to the other ones along the minor axis for the majority of them. The exceptions are for Fe4383, Fe4531, $\mathrm{Fe} 4668$ and marginally $\mathrm{H} \beta$. Considering both directions and the uncertainties of the gradients, the majority of the iron indices are zero or positive (Fe4531, Fe5270, Fe5335, 〈Fe $\rangle, \mathrm{Fe} 5709$ and Fe5782), while the $\mathrm{Mgb}$ gradient is zero and the $\mathrm{Mg}_{1}$ and $\mathrm{Mg}_{2}$ gradients are negative. However, the gradients of Fe4383, $\mathrm{Fe} 4668$ and Fe5406 are still negative. The Ca4455 gradient is 
Table 18. Radial gradients along the major and minor axes of both galaxies respectively for each Lick index.

\begin{tabular}{|c|c|c|c|c|}
\hline \multicolumn{5}{|c|}{ Gradients and Linear constants } \\
\hline \multirow[t]{2}{*}{ Lick Index } & NGC 1052 & & NGC 7796 & \\
\hline & $\left(\AA \operatorname{dex}^{-1}\right)$ & 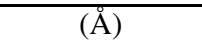 & $\left(\AA \operatorname{dex}^{-1}\right)$ & $(\AA)$ \\
\hline \multirow[t]{2}{*}{$\mathrm{Fe} 4383$} & $-0.81 \pm 0.24$ & $5.16 \pm 0.26$ & $-0.44 \pm 0.39$ & $4.65 \pm 0.38$ \\
\hline & $-2.04 \pm 0.64$ & $3.99 \pm 0.59$ & $-2.17 \pm 0.50$ & $3.09 \pm 0.47$ \\
\hline \multirow[t]{2}{*}{ Fe4531 } & $-0.29 \pm 0.24$ & $3.25 \pm 0.26$ & $+0.72 \pm 0.39$ & $4.31 \pm 0.38$ \\
\hline & $-1.57 \pm 0.64$ & $1.70 \pm 0.59$ & $-0.92 \pm 0.50$ & $1.99 \pm 0.47$ \\
\hline \multirow[t]{2}{*}{ Fe4668 } & $-1.34 \pm 0.24$ & $6.80 \pm 0.26$ & $-1.73 \pm 0.39$ & $6.52 \pm 0.38$ \\
\hline & $-5.15 \pm 0.64$ & $3.08 \pm 0.59$ & $-5.80 \pm 0.50$ & $0.97 \pm 0.47$ \\
\hline \multirow[t]{3}{*}{ Fe5270 } & $+0.38 \pm 0.24$ & $3.00 \pm 0.26$ & $+0.34 \pm 0.39$ & $3.33 \pm 0.38$ \\
\hline & $-0.67 \pm 0.64$ & $1.82 \pm 0.59$ & $+0.67 \pm 0.50$ & $3.32 \pm 0.47$ \\
\hline & $-0.56 \pm 0.27$ & - & - & - \\
\hline \multirow[t]{2}{*}{ Fe5335 } & $-0.23 \pm 0.24$ & $2.20 \pm 0.26$ & $+0.53 \pm 0.39$ & $3.19 \pm 0.38$ \\
\hline & $-0.98 \pm 0.64$ & $1.78 \pm 0.59$ & $+0.06 \pm 0.50$ & $2.75 \pm 0.47$ \\
\hline \multirow[t]{2}{*}{$\langle\mathrm{Fe}\rangle$} & $+0.07 \pm 0.23$ & $2.61 \pm 0.25$ & $+0.44 \pm 0.39$ & $3.26 \pm 0.38$ \\
\hline & $-0.82 \pm 0.64$ & $1.80 \pm 0.59$ & $+0.37 \pm 0.73$ & $3.04 \pm 0.69$ \\
\hline \multirow[t]{2}{*}{ Fe5406 } & $-0.11 \pm 0.24$ & $1.77 \pm 0.26$ & $-0.63 \pm 0.39$ & $0.94 \pm 0.38$ \\
\hline & $-0.17 \pm 0.64$ & $1.81 \pm 0.59$ & $-0.64 \pm 0.50$ & $0.90 \pm 0.47$ \\
\hline \multirow[t]{2}{*}{ Fe5709 } & $+0.12 \pm 0.24$ & $1.19 \pm 0.26$ & $-0.50 \pm 0.50$ & $0.41 \pm 0.53$ \\
\hline & $+0.26 \pm 0.64$ & $1.23 \pm 0.59$ & $-0.34 \pm 0.50$ & $0.49 \pm 0.47$ \\
\hline \multirow[t]{2}{*}{ Fe5782 } & $-0.30 \pm 0.24$ & $0.45 \pm 0.26$ & $-0.41 \pm 0.39$ & $0.49 \pm 0.38$ \\
\hline & $-0.10 \pm 0.64$ & $1.12 \pm 0.59$ & $-0.34 \pm 0.50$ & $0.49 \pm 0.47$ \\
\hline \multirow[t]{2}{*}{ Ca4455 } & $0.00 \pm 0.49$ & $2.44 \pm 0.50$ & $-0.10 \pm 0.80$ & $2.51 \pm 0.74$ \\
\hline & $-0.51 \pm 1.08$ & $1.97 \pm 0.95$ & $-0.89 \pm 0.91$ & $1.84 \pm 0.78$ \\
\hline \multirow[t]{2}{*}{$\mathrm{Mg} \mathrm{b}$} & $-0.38 \pm 0.18$ & $4.44 \pm 0.19$ & $-0.18 \pm 0.58$ & $4.93 \pm 0.55$ \\
\hline & $-0.28 \pm 0.89$ & $4.63 \pm 0.80$ & $-0.63 \pm 0.70$ & $4.32 \pm 0.64$ \\
\hline \multirow[t]{3}{*}{$\mathrm{Na} \mathrm{D}$} & $-2.34 \pm 0.19$ & $2.47 \pm 0.20$ & $-0.81 \pm 0.31$ & $3.98 \pm 0.30$ \\
\hline & $-4.60 \pm 0.49$ & $0.73 \pm 0.45$ & $-0.31 \pm 0.39$ & $4.24 \pm 0.36$ \\
\hline & $-4.01 \pm 0.14$ & - & - & - \\
\hline \multirow[t]{2}{*}{$\mathrm{H} \beta$} & - & - & $-0.17 \pm 0.40$ & $1.35 \pm 0.38$ \\
\hline & $\begin{array}{c}- \\
\left(\operatorname{mag} \operatorname{dex}^{-1}\right)\end{array}$ & $\begin{array}{c}- \\
\text { (mag) }\end{array}$ & $\begin{array}{c}+0.38 \pm 0.50 \\
\left(\operatorname{mag~dex~}^{-1}\right)\end{array}$ & $\begin{array}{c}1.68 \pm 0.47 \\
(\mathrm{mag})\end{array}$ \\
\hline \multirow[t]{3}{*}{$\mathrm{Mg}_{1}$} & $-0.028 \pm 0.004$ & $0.132 \pm 0.004$ & $-0.018 \pm 0.011$ & $0.149 \pm 0.010$ \\
\hline & $-0.015 \pm 0.016$ & $0.162 \pm 0.014$ & $-0.024 \pm 0.013$ & $0.146 \pm 0.011$ \\
\hline & $-0.074 \pm 0.004$ & - & - & - \\
\hline \multirow[t]{5}{*}{$\mathrm{Mg}_{2}$} & $-0.007 \pm 0.004$ & $0.304 \pm 0.004$ & $-0.031 \pm 0.011$ & $0.312 \pm 0.010$ \\
\hline & $+0.073 \pm 0.017$ & $0.400 \pm 0.016$ & $-0.043 \pm 0.013$ & $0.307 \pm 0.012$ \\
\hline & $-0.074 \pm 0.004$ & - & - & - \\
\hline & $-0.061 \pm 0.019$ & - & - & - \\
\hline & $-0.068 \pm 0.029$ & - & - & - \\
\hline
\end{tabular}

Columns 3 and 5: the linear regression constant of the fitting that represents the Lick index at one effective radius.

For Fe5270, Na D, $\mathrm{Mg}_{1}$ and $\mathrm{Mg}_{2}$, the third line shows their gradients measured in NGC 1052 by Carollo et al. (1993).

For $\mathrm{Mg}_{2}$ only, the fourth and fifth lines show the gradients measured respectively along the major and minor axis of NGC 1052 by Couture \& Hardy (1988).

zero. The Na D gradient is negative. The Na D can be contaminated by its own internal interstellar absorption of NGC 7796, like NGC 1052. Due to the contribution of molecular lines in $\mathrm{Fe} 4383$ and $\mathrm{Fe} 4668$, a negative gradient of the carbon stellar abundance can also be proposed for NGC 7796. The mean $\mathrm{H} \beta$ gradient is also zero.

In order to derive the radial variations of the abundance ratios, we have analyzed the index variations directly on the index-index planes, where the direction of the vector age-metallicity-abundance ratio can be modeled properly (see Sect. 5). The stellar population synthesis helps to disentangle the age-metallicity degeneracy of the Lick indices as well (see Sect. 6).

\subsection{External comparisons of some radial gradients}

Couture \& Hardy (1988) have measured the $\mathrm{Mg}_{2}$ gradient along both axes of NGC 1052 and their data are plotted in comparison with ours in Fig. 7. The agreement is marginal because the errors of the Couture \& Hardy (1988) measurements are greater. The $\mathrm{Mg}_{2}$ gradients measured by them are different to ours (see Table 18). The same exists when a comparison is made with the $\mathrm{Mg}_{2}$ gradient measured by Carollo et al. (1993) along the E-W direction. Carollo et al. (1993) have also computed the gradients of Fe5270, $\mathrm{NaD}$ and $\mathrm{Mg}_{1}$ along the same direction. Despite some agreement for the radial variation of them in the plots of Fig. 5 (Fe5270), Fig. 6 ( $\mathrm{Na} \mathrm{D}$ ) and Fig. 7 ( $\mathrm{Mg}_{1}$ and $\left.\mathrm{Mg}_{2}\right)$, the computed gradients are different to ours (Table 18). For $\mathrm{Mg}_{1}$ 

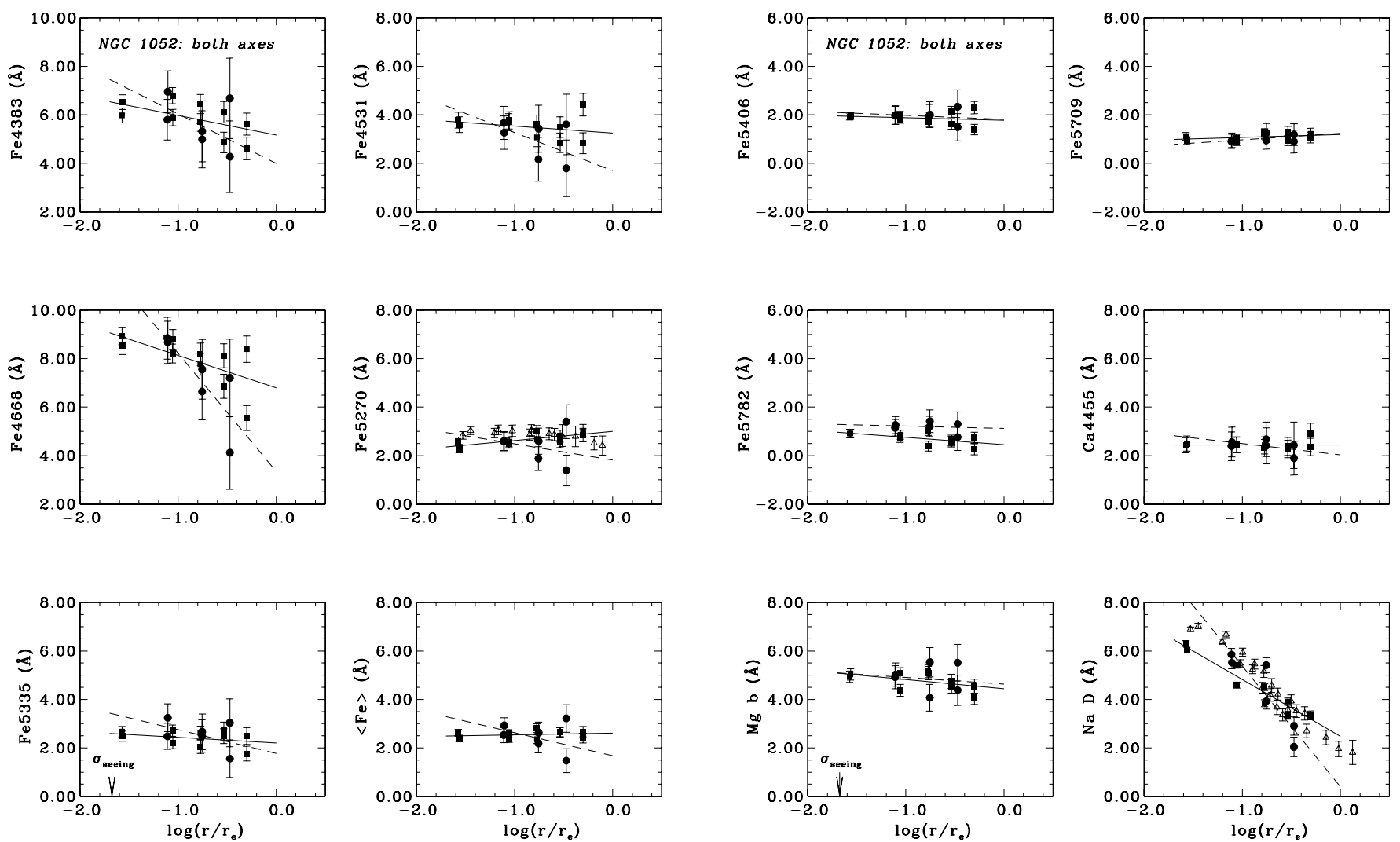

Fig. 5. Radial gradients of Fe4383, Fe4531, Fe4668, Fe5270, Fe5335 and $\langle\mathrm{Fe}\rangle$ along both axes of NGC 1052 whose respective linear regressions are shown by solid and dashed lines for the major (solid squares) and minor axis (solid circles) data respectively. The seeing size $\sigma_{\text {seeing }}$ is given on the abscissa of the bottom-left plot $\left(\sigma_{\text {seeing }}=\right.$ $F W H M_{\text {seeing }} / 2.355$ ). The Fe5270 data of Carollo et al. (1993) along the E-W direction, open triangles, are also plotted.

and $\mathrm{Mg}_{2}$ only, Carollo et al. (1993) should not have applied emission line corrections.

No radial gradient of the Lick index has been quantified for NGC 7796. Bertin et al. (1994) have measured the radial profile of $\mathrm{Mg}_{2}$ along the major axis whose direct comparison with our data is presented in Fig. 10. There is a systematic difference between the Bertin et al. (1994) data and our measurements ( $\sim 0.113 \mathrm{mag}$ ) although the $\mathrm{Mg}_{2}$ gradient computed using their data is very close to our result: $-0.031 \pm 0.013 \operatorname{mag~dex}^{-1}$. The predicted $\mathrm{Mg}_{2}^{0}$ for NGC 7796 by the $\mathrm{Mg}_{2}^{0}-\sigma_{v}^{0}$ relation of Bender et al. (1993) is greater than that using the observations of Bertin et al. (1994).

\section{Comparisons with single-aged stellar population models}

The single-aged stellar population (SSP) models of Thomas et al. (2003a), hereafter TMB (2003a), have been adopted in order to make predictions about the spatial distribution of the stellar populations inside the observed regions of each galaxy in terms of chemical abundances and age. We have made direct comparisons of the measured Lick indices of each aperture spectrum with the theoretical ones of these SSP models.

The SSP models of TMB (2003a) are built using the evolutive stellar population synthesis of Maraston (1998). The main characteristic of the code of TMB (2003a) is to compute the influence of the abundance variations on the Lick indices, although
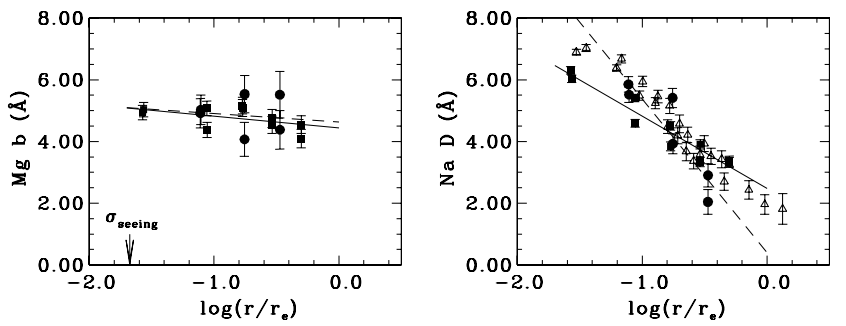

Fig. 6. Radial gradients of Fe5406, Fe5709, Fe5782, Ca4455, Mg b and $\mathrm{NaD}$ along both axes of NGC 1052. The notation is the same as in Fig. 5. The Na D data of Carollo et al. (1993) along the E-W direction, open triangles, are also plotted.

this code uses the evolutive models of Maraston (1998) which are essentially based on the solar abundance ratios. The Lick indices of these SSP models are computed adopting the stellar fitting functions of Worthey (1994) and the response functions of Tripicco \& Bell (1995). The abundance variations are only considered for the alpha-elements like magnesium.

The TMB (2003a) SSP models assuming the IMF of Salpeter (1955) have been used. We have used the SSP models with five ages $(2,6,10,12$ and $15 \mathrm{Gyr})$, six global metallicities $\left(\left[Z / Z_{\odot}\right]=\right.$ $-2.25,-1.35,-0.33,0.00,+0.35,+0.67 \mathrm{dex})$ and four alphaelement to iron ratios $([\alpha / \mathrm{Fe}]=-0.3,0.0,+0.3,+0.5 \mathrm{dex})$.

We have plotted, as planes Lick index versus Lick index, our data of all aperture spectra of NGC 1052 and NGC 7796 with the theoretical ones of TMB's SSP models.

For NGC 1052, Figs. 11a,b, 12a,b and 13a,b show the $\mathrm{Mg} \mathrm{b}$ index plotted versus iron index: $\mathrm{Mgb}$ versus $\mathrm{Fe} 4531$ (Fig. 11a), Mg b versus Fe5270 (Fig. 11b), Mg b versus Fe5335 (Fig. 12a), Mg b versus Fe5406 (Fig. 12b), Mg b versus Fe5709 (Fig. 13a) and Mgb versus Fe5782 (Fig. 13b). Figures 14a and $14 \mathrm{~b}$ show $\mathrm{Mg}_{1}$ versus $\langle\mathrm{Fe}\rangle$ and $\mathrm{Mg}_{2}$ versus $\langle\mathrm{Fe}\rangle$ respectively. Figure 15 shows $\mathrm{NaD}$ versus $\langle\mathrm{Fe}\rangle$.

The $\mathrm{Ca} 4455, \mathrm{Fe} 4383$ and $\mathrm{Fe} 4668$ indices were not adopted because they are blended with other absorption lines: Ca4455 with atomic lines of Fe and $\mathrm{Cr}$ as TMB (2003a), and Fe4383 and Fe4668 with molecular lines of $\mathrm{C}_{2}$ and $\mathrm{CH}$ respectively.

For NGC 7796, Figs. 17a,b, 18a,b and 19a,b show respectively the same plots of $\mathrm{Mgb}$ index versus an iron index as for NGC 1052. Figures 20a and 20b show $\mathrm{Mg}_{1}$ versus $\langle\mathrm{Fe}\rangle$ and $\mathrm{Mg}_{2}$ versus $\langle\mathrm{Fe}\rangle$ respectively. Figure 21 shows $\mathrm{NaD}$ versus $\langle\mathrm{Fe}\rangle$ respectively. 

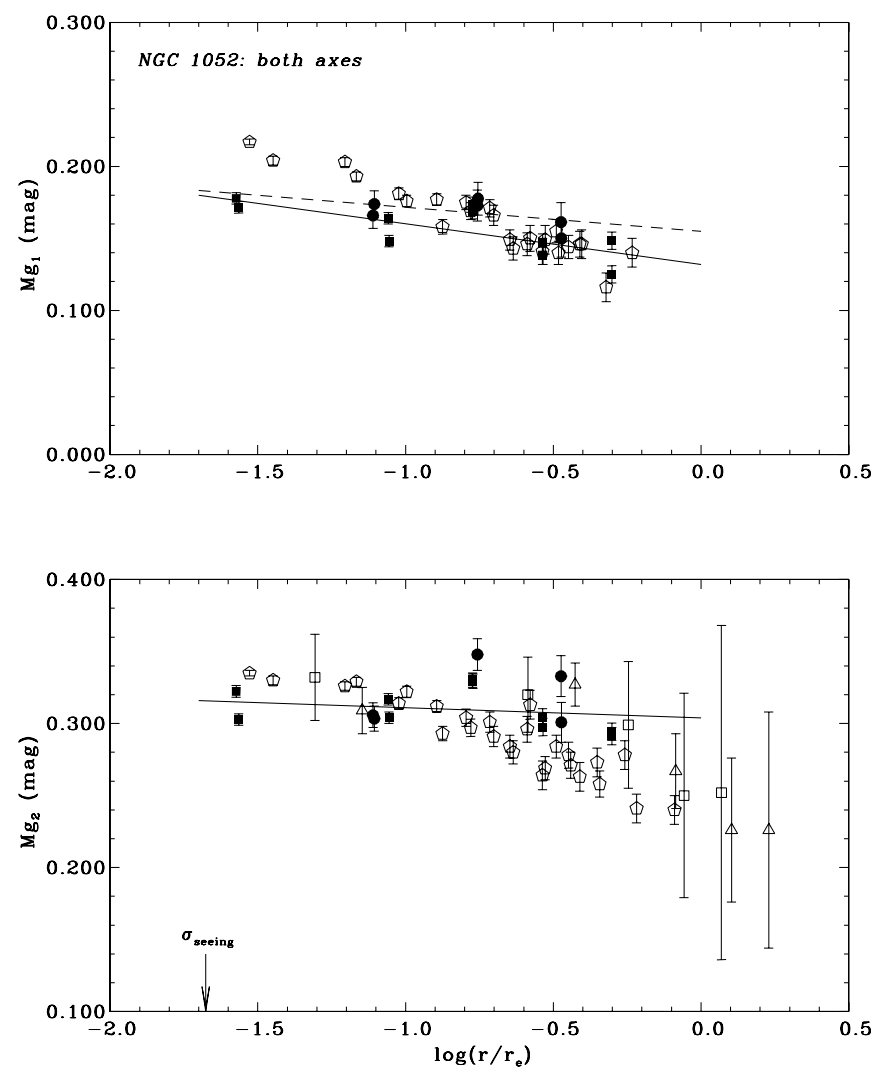

Fig. 7. Radial gradients of $\mathrm{Mg}_{1}$ and $\mathrm{Mg}_{2}$ along both axes of NGC 1052 . The notation is the same as in Fig. 5. The fit for the minor axis $\mathrm{Mg}_{2}$ data is not plotted. The $\mathrm{Mg}_{2}$ data of Couture \& Hardy (1988) along the major and minor axis, open squares and open triangles respectively, are also plotted (bottom panel). The $\mathrm{Mg}_{1}$ and $\mathrm{Mg}_{2}$ data of Carollo et al. (1993) along the E-W direction, open pentagons, are plotted (both panels).

The plots present an element abundance indicator $(\mathrm{Mgb}$, $\mathrm{Mg}_{1}$ and $\mathrm{Mg}_{2}$ for magnesium and $\mathrm{NaD}$ for sodium) versus an iron abundance indicator (Fe4531, Fe5270, Fe5335, Fe5408, $\mathrm{Fe} 5709, \mathrm{Fe} 5782$ and $\langle\mathrm{Fe}\rangle)$. In all these plots, the predicted Lick indices of the SSPs are localized in four curve families, each one with a different $\alpha$-element/Fe ratio. Each curve family presents Lick indices of the SSPs with five ages and six metallicities that are distributed with very small dispersion in some restricted area (the age-metallicity degeneracy of Lick indices). The present index errors correspond to a mean imprecision of \pm 0.1 dex for the $[\alpha / \mathrm{Fe}]$ determination in that plots.

Figure 16 shows $[\mathrm{MgFe}]_{\mathrm{TMB}}$ modified Lick (TMB 2003a) index versus $\mathrm{H} \beta$ for NGC 1052 using the data of the more external spectra only. Figure 22 presents the same for NGC 7796 using all data. Both figures are discussed later. The $[\mathrm{MgFe}]_{\mathrm{TMB}}$ index of TMB (2003a) is a metallicity indicator: $[\mathrm{MgFe}]_{\mathrm{TMB}}=$ $\sqrt{\mathrm{Mg} \mathrm{b} \times(0.72 \times \mathrm{Fe} 5270+0.28 \times \mathrm{Fe} 5335)}$. This index is insensitive to the $\alpha / \mathrm{Fe}$ ratio. The $\mathrm{H} \beta$ Lick index is a stellar age indicator for SSPs with 2 Gyr or more.

For NGC 1052, we have obtained the following stellar population analysis using the Lick indices of all extracted spectra along both photometric axes.

The stellar populations inside the observed region of NGC 1052 present a variable alpha-enhancement (between the solar ratio and $[\alpha / \mathrm{Fe}]=+0.5 \mathrm{dex})$. The luminosity-weighted mean global metallicity seems to change in the interval from $\left[Z / Z_{\odot}\right]=0.0$ to +0.35 dex. No information about age can be obtained from these plots. No plots of $\mathrm{Mgb}$ vs. iron index show a monotonic radial dependency for the $\alpha / \mathrm{Fe}$ ratio.
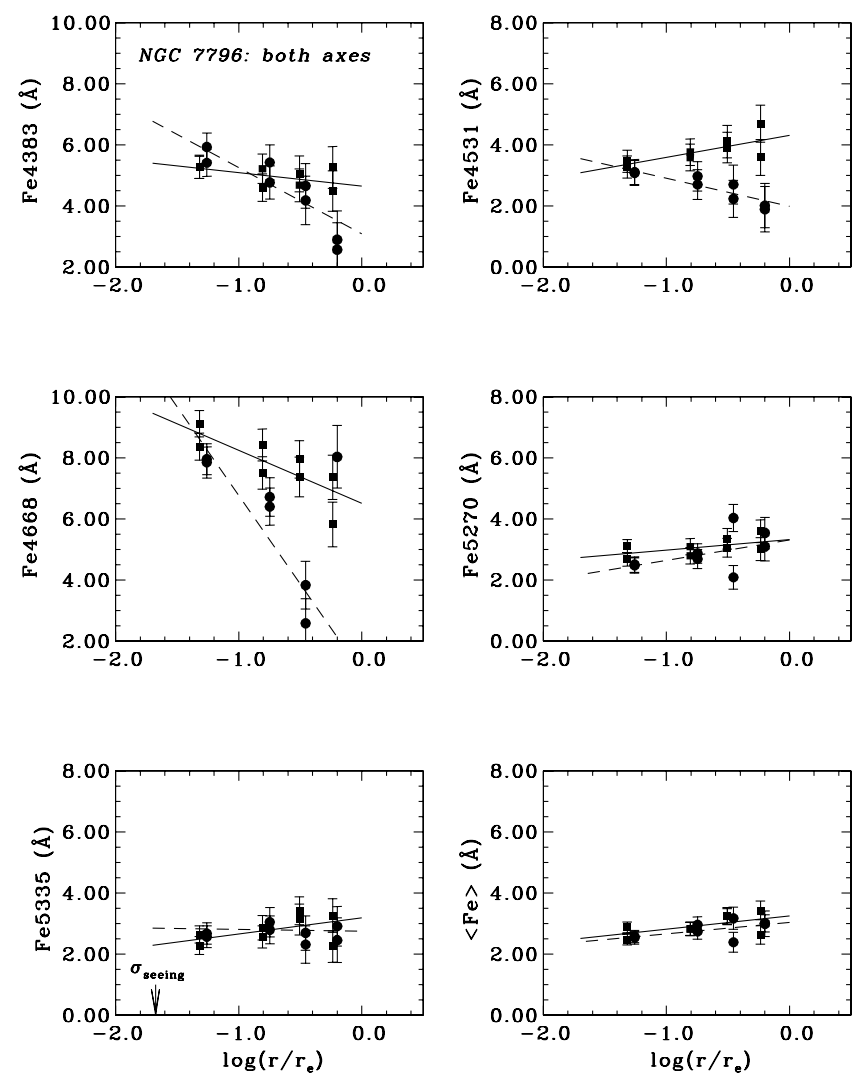

Fig. 8. Radial gradients of Fe4383, Fe4531, Fe4668, Fe5270, Fe5335 and $\langle\mathrm{Fe}\rangle$ along both axes of NGC 7796. The notation is the same as in Fig. 5.
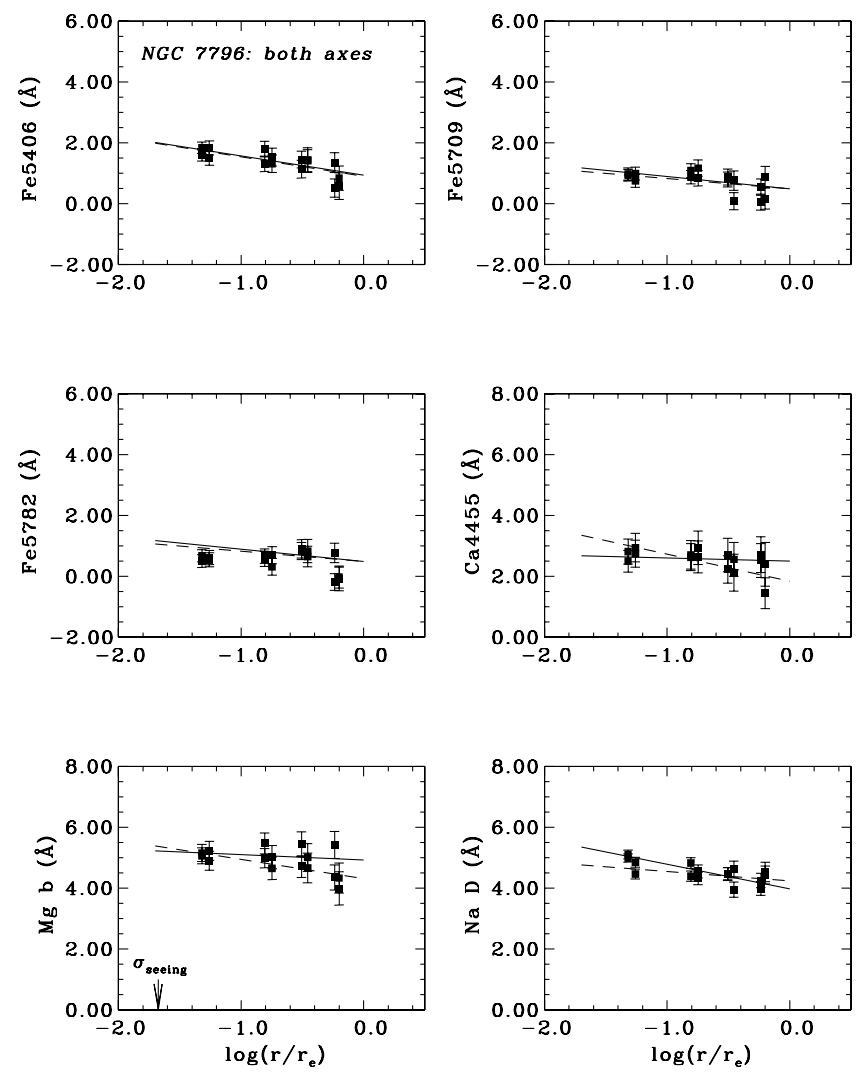

Fig. 9. Radial gradients of Fe5406, Fe5709, Fe5782, Ca4455, Mg b and $\mathrm{NaD}$ along both axes of NGC 7796. The notation is the same as in Fig. 5. 

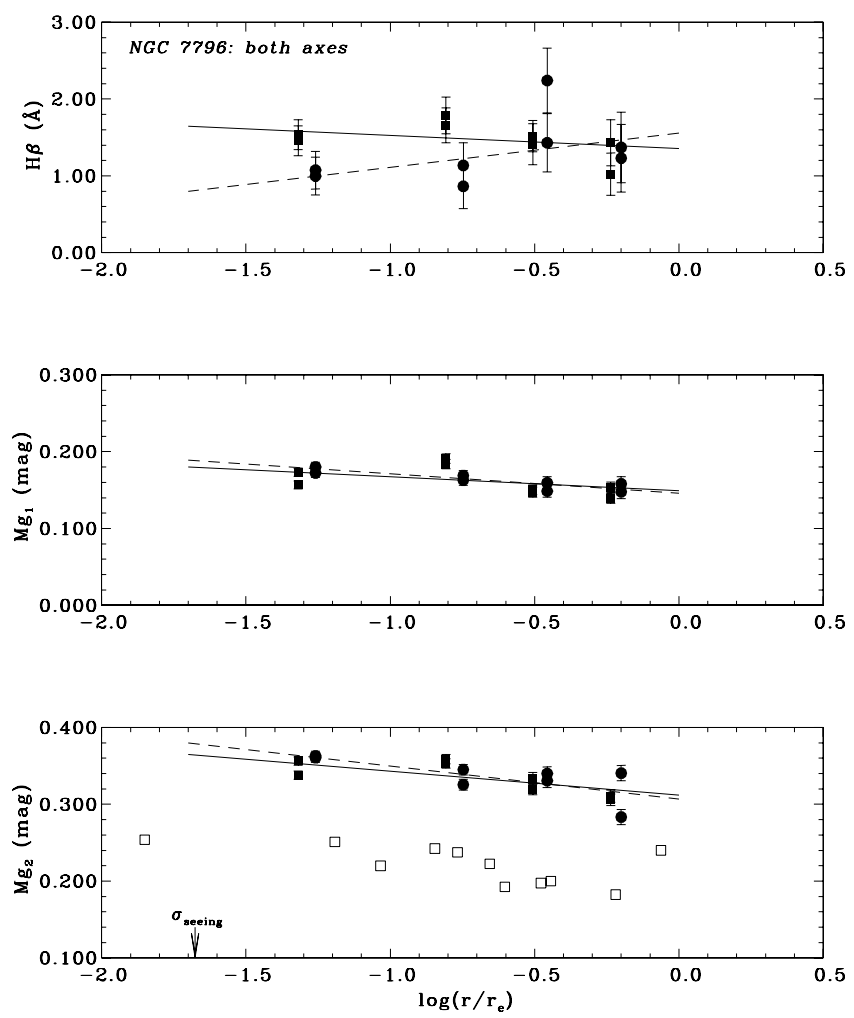

Fig. 10. Radial gradients of $\mathrm{H} \beta, \mathrm{Mg}_{1}$ and $\mathrm{Mg}_{2}$ along both axes of NGC 7796. The notation is the same as in Fig. 5. The $\mathrm{Mg}_{2}$ data of Bertin et al. (1994) along the major, open squares, are also plotted (bottom panel).

On the other hand, the data of the central extracted spectrum indicate a little oversolar $\alpha / \mathrm{Fe}$ ratio (luminosity-averaged $[\alpha / \mathrm{Fe}] \approx$ $+0.2 \pm 0.2 \mathrm{dex}$ ) or nearly solar, taking into account its error.

Therefore, the timescale of the star formation in the nucleus was $1 \mathrm{Gyr}$ if we adopt the relation between the $\alpha / \mathrm{Fe}$ ratio and timescale from Thomas et al. (2005); see Sect. 7. Nearly the same timescale is obtained for the observed region along the minor axis direction, i.e. the bulge. However, the strong spread of the $\mathrm{Mg} / \mathrm{Fe}$ ratio along the major axis or disc shows a great dispersion of the star formation timescale based on an unknown galaxy formation process. Therefore, in the disc of NGC 1052 the chemical enrichment of $\alpha$-elements by SN-II relative to the iron elements by $\mathrm{SN}-\mathrm{Ia}$ has happened in a heterogeneous way. No information about the star ages can be obtained because the $\mathrm{H} \beta$ index is completely modified by nebular emission of this Liner.

The relation between the global metallicity, the iron abundance and the $\alpha / \mathrm{Fe}$ ratio by Salaris et al. (1993) can be used to estimate one value as a function of the other ones and to understand the interconnected variations of these quantities. For example, the constant value of the global metallicity with the increasing of the $\alpha / \mathrm{Fe}$ ratio together with the decreasing of the iron abundance, and a decreasing of $Z$ due to a decreasing of $\alpha / \mathrm{Fe}$ are explained by this relation.

$\left[Z / Z_{\odot}\right]=[\mathrm{Fe} / \mathrm{H}]+\log \left(0.638 \times 10^{[\alpha / \mathrm{Fe}]}+0.362\right)$.

In the next paragraphs, we show the analysis in each index versus index plot for NGC 1052.

The Lick index data obtained along both axes are reasonably distributed in the same regions of the index vs. index planes.

The $\mathrm{Mg}$ b versus Fe4531 plot (Fig. 11a) and the $\mathrm{Mg}$ b versus Fe5782 plot (Fig. 13b) show the observed Lick indices of the extracted spectra with a distribution between the curve families
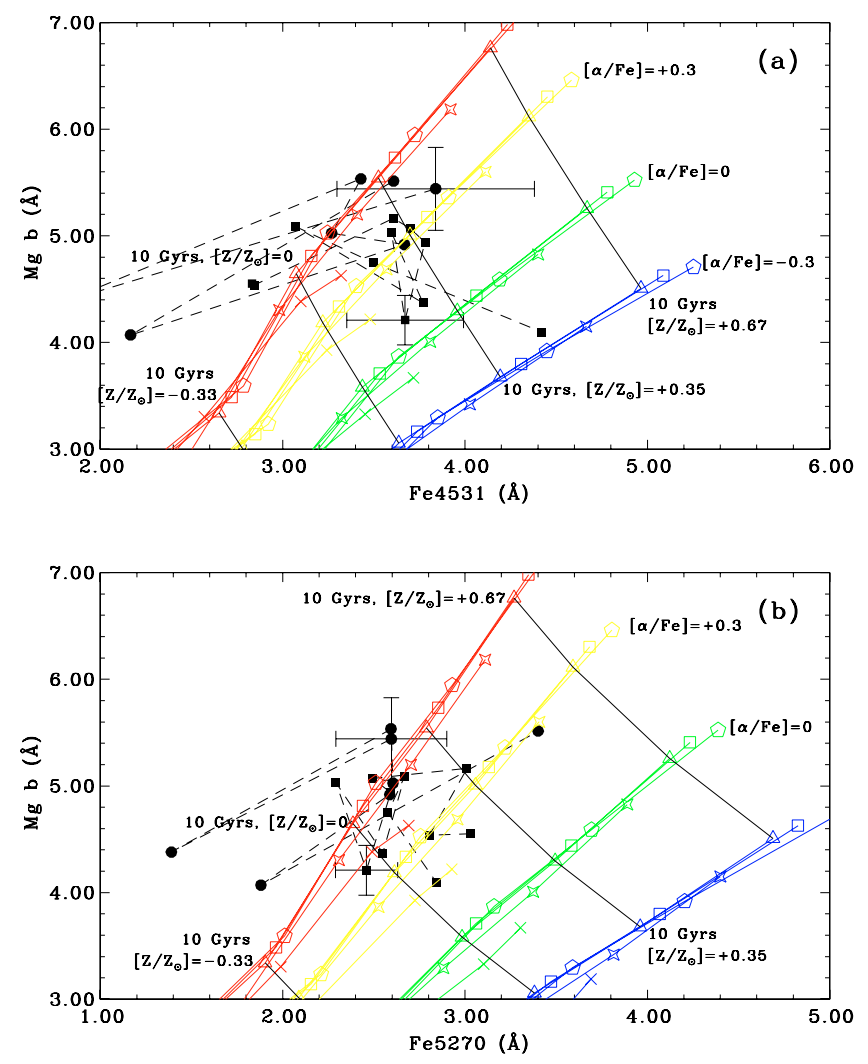

Fig. 11. a) $\mathrm{Mg}$ b versus Fe4531 plot (top panel); b) $\mathrm{Mg}$ b versus Fe5270 plot (bottom panel): comparisons of Lick indices of the long slit spectra of NGC 1052 (both photometric axes) with the theoretical ones of TMB (2003a)'s SSPs. The index values of the spectra are represented by solid black squares for the major axis data and solid black circles for the minor axis data, which are both connected by traced black lines. The index errors are only plotted for the central spectrum data. The SSP predicted index values are represented by open symbols whose colour indicate the curve families with different $[\alpha / \mathrm{Fe}]:-0.3$ (blue), 0.0 (green), +0.3 (yellow) and $+0.5 \mathrm{dex}$ (red). The SSP ages are represented by open symbols: 2 (crosses), 6 (four-points stars), 10 (triangles), 12 (squares) and $15 \mathrm{Gyr}$ (pentagons). The global metallicities, $\left[Z / Z_{\odot}\right]=-2.25,-1.35$, $-0.33,0.00,+0.35,+0.67 \mathrm{dex}$, increase from the left-bottom to the right-top for a given $[\alpha / \mathrm{Fe}]$ family curve. The predicted Lick indices of the SSPs with $10 \mathrm{Gyr}$ and different $[\alpha / \mathrm{Fe}]$ are connected by solid black lines for a specific metallicity.

of the SSPs with $[\alpha / \mathrm{Fe}]=0.0$ and +0.5 dex in the region of the metallicities $\left[Z / Z_{\odot}\right]=0.00$ and $+0.35 \mathrm{dex}$.

The $\mathrm{Mg}$ b versus Fe5270 plot (Fig. 11b) and the Mg b versus Fe5335 plot (Fig. 12a) show the indices of the spectra with a distribution between the curves of the SSPs with $[\alpha / \mathrm{Fe}]=$ +0.3 (or below) and +0.5 dex in the region of $\left[Z / Z_{\odot}\right]=0.00$ and +0.35 dex.

The $\mathrm{Mg} \mathrm{b}$ versus Fe5406 plot (Fig. 12b) and the Mg b versus Fe5709 plot (Fig. 13a) show the indices of the spectra with a distribution between the curves of the SSPs with $[\alpha / \mathrm{Fe}]=0.0$ and +0.3 dex in the region of $\left[Z / Z_{\odot}\right]=+0.35 \mathrm{dex}$.

The $\mathrm{Mg}_{1}$ versus $\langle\mathrm{Fe}\rangle$ plot (Fig. 14a) and the $\mathrm{Mg}_{2}$ versus $\langle\mathrm{Fe}\rangle$ plot (Fig. 14b) show the indices of the spectra with a distribution between the curves of the SSPs with $[\alpha / \mathrm{Fe}]=+0.3$ and $+0.5 \mathrm{dex}$ in the region of $\left[Z / Z_{\odot}\right]=0.00$ and $+0.35 \mathrm{dex}$.

The NaD versus $\langle\mathrm{Fe}\rangle$ plot (Fig. 15) shows the indices of the spectra with a distribution between the curves of the SSPs with $[\alpha / \mathrm{Fe}]=+0.3$ and $+0.5 \mathrm{dex}$ (or higher) at the region of $\left[Z / Z_{\odot}\right]=+0.35 \mathrm{dex}$, where the data of more central spectra are localized above the curve families with highest $\alpha / \mathrm{Fe}$ 

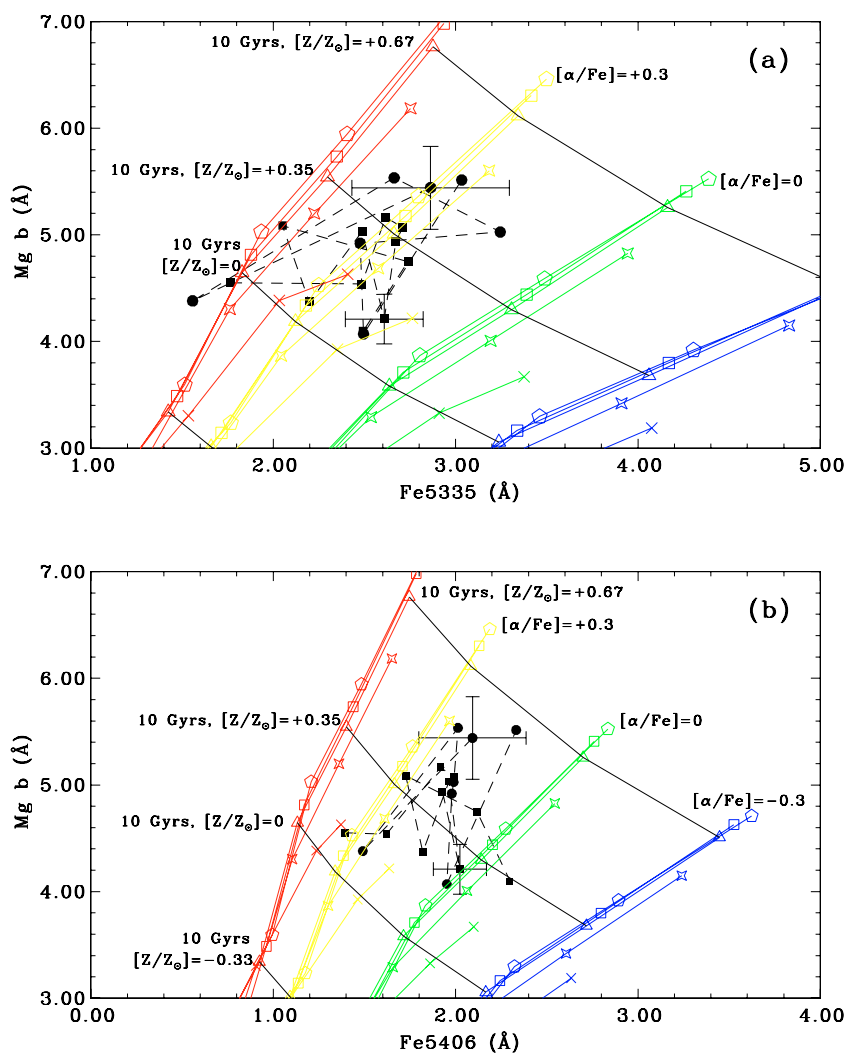

Fig. 12. a) $\mathrm{Mg}$ b versus Fe5335; $\mathrm{Mg}$ b versus Fe5406 b): comparisons of the observed Lick indices of the aperture spectra of NGC 1052 with the theoretical ones of TMB (2003a)'s SSPs. The notation is the same as in Fig. 11.

ratio. However, $\mathrm{Na}$ is not an $\alpha$-element. Moreover, the interstellar medium can increase the values of the $\mathrm{Na} \mathrm{D}$ index. Therefore, the overabundance of sodium relative to iron is still an open question.

The $[\mathrm{MgFe}]_{\mathrm{TMB}}$ versus $\mathrm{H} \beta$ plot for NGC 1052 (Fig. 16) was made using the data along the major axis $(6.8$ arcsec $\geq r \geq$ 20.2 arcsec). The data of NGC 1052 ( $r \geq 11.8$ arcsce) are concentrated outside of the region of the theoretical Lick indices of the SSPs, i.e. in the region of values of $\mathrm{H} \beta$ lower than $1 \AA$, however near of the curves with $\left[Z / Z_{\odot}\right]=0.0$ dex and the highest ages. This indicates that there still is a gas emission contribution to the $\mathrm{H} \beta$ line in the outer regions. The stellar ages in these regions are undetermined through the analysis of this Balmer line.

For NGC 7796, we have performed the following stellar population analysis using the Lick indices of all extracted spectra along both photometric axes.

The stellar populations inside the observed region of NGC 7796 present a variable high alpha-enhancement (maybe between the solar ratio and $[\alpha / \mathrm{Fe}]=+0.5 \mathrm{dex})$. However, differently from NGC 1052, the plots of Mgb versus Fe5270 (Fig. 17b), Mg b vs. Fe5335 (Fig. 18a), Mg $_{1}$ vs. $\langle\mathrm{Fe}\rangle$ (Fig. 20a) and $\mathrm{Mg}_{2}$ vs. $\langle\mathrm{Fe}\rangle$ (Fig. 20b) show a radial dependency for the luminosity-weighted mean $\alpha / \mathrm{Fe}$ ratio that seems to be greater in the nucleus $([\alpha / \mathrm{Fe}] \approx+0.44 \pm 0.09 \mathrm{dex})$ than in the outer regions. The stellar populations in the observed region of NGC 7796 have a luminosity-averaged global metallicity between $\left[Z / Z_{\odot}\right]=0.0$ and +0.35 dex, or almost around +0.35 dex, with ages from 6 to 12 Gyr (see Fig. 22).

Therefore, the timescale of star formation in the nucleus was nearly 0.04 Gyr adopting the relation of Thomas et al. (2005);
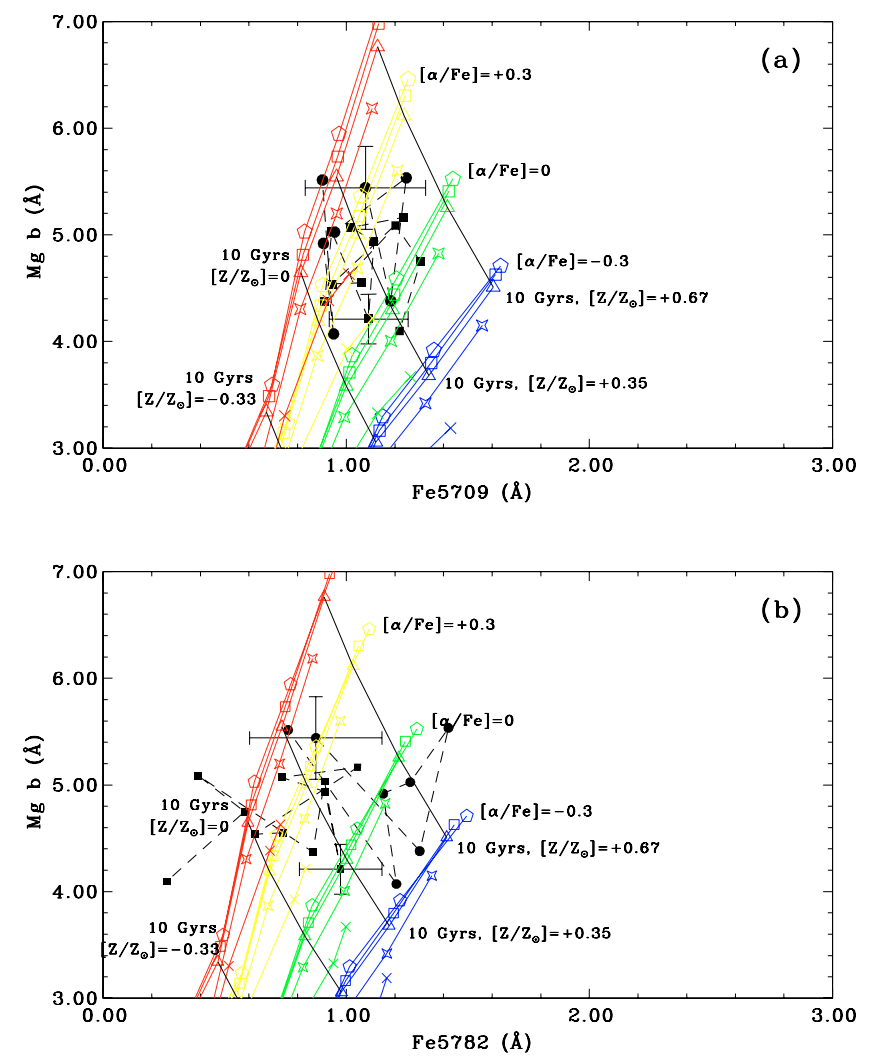

Fig. 13. a) $\mathrm{Mg}$ b versus $\mathrm{Fe} 5709$; b) $\mathrm{Mg}$ b versus Fe5782: comparisons of the observed Lick indices of the aperture spectra of NGC 1052 with the theoretical ones of TMB (2003a)'s SSPs. The notation is the same as in Fig. 11.
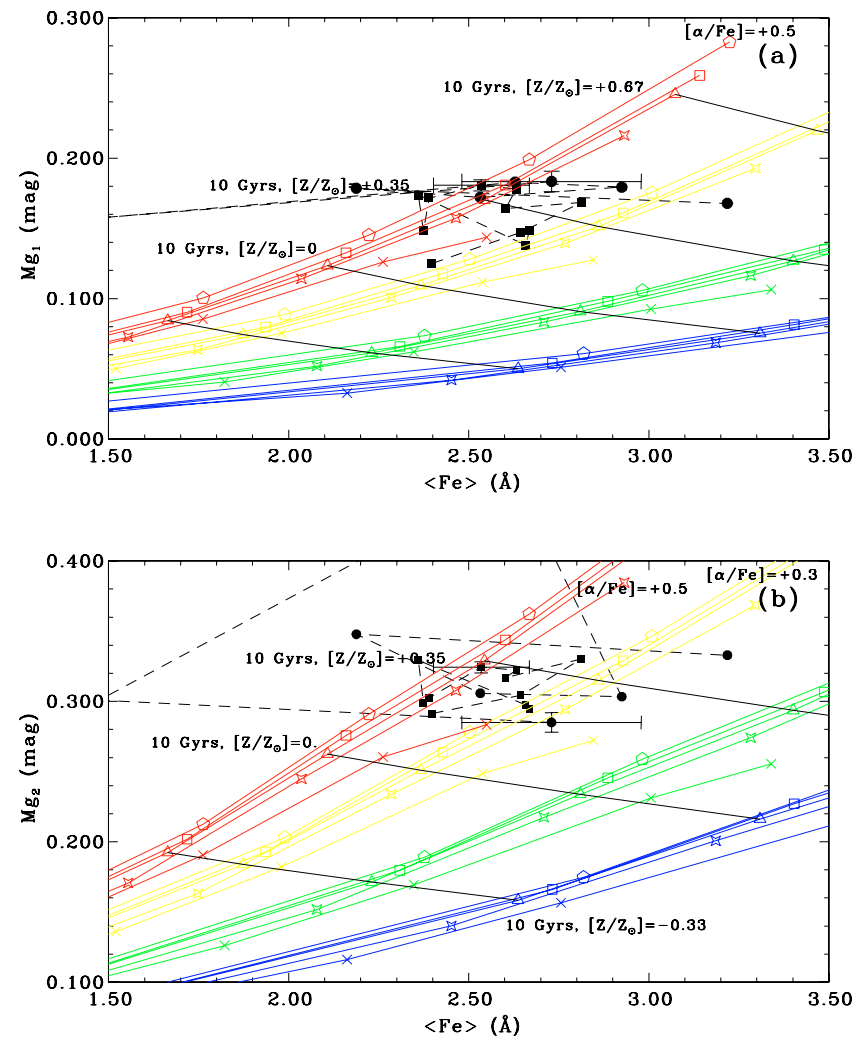

Fig. 14. a) $\mathrm{Mg}_{1}$ versus $\langle\mathrm{Fe}\rangle$; b) $\mathrm{Mg}_{2}$ versus $\langle\mathrm{Fe}\rangle$ : comparisons of the observed Lick indices of the aperture spectra of NGC 1052 with the theoretical ones of TMB (2003a)'s SSPs. The notation is the same as in Fig. 11. 


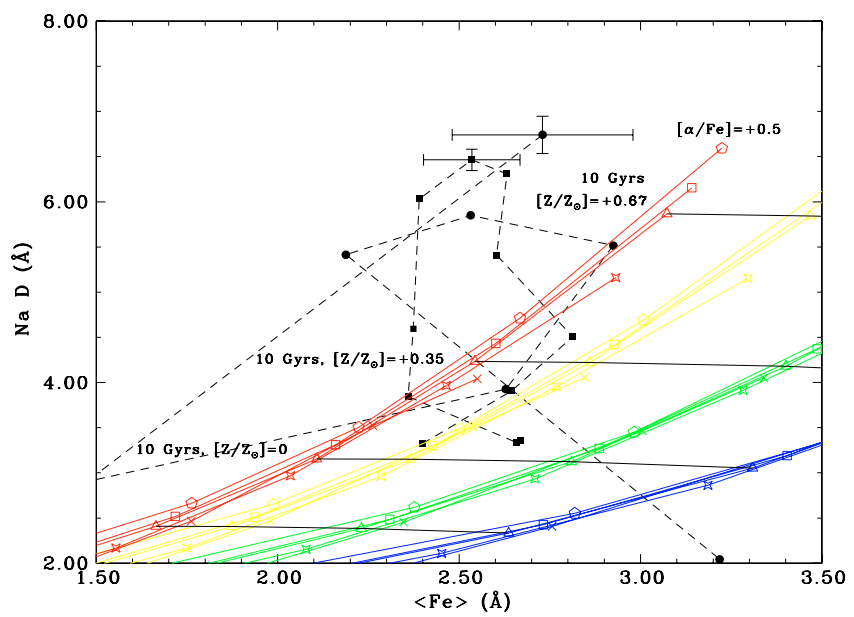

Fig. 15. Na D versus $\langle\mathrm{Fe}\rangle$ : comparisons of the observed Lick indices of the aperture spectra of NGC 1052 with the theoretical ones of TMB (2003a)'s SSPs. The notation is the same as in Fig. 11.

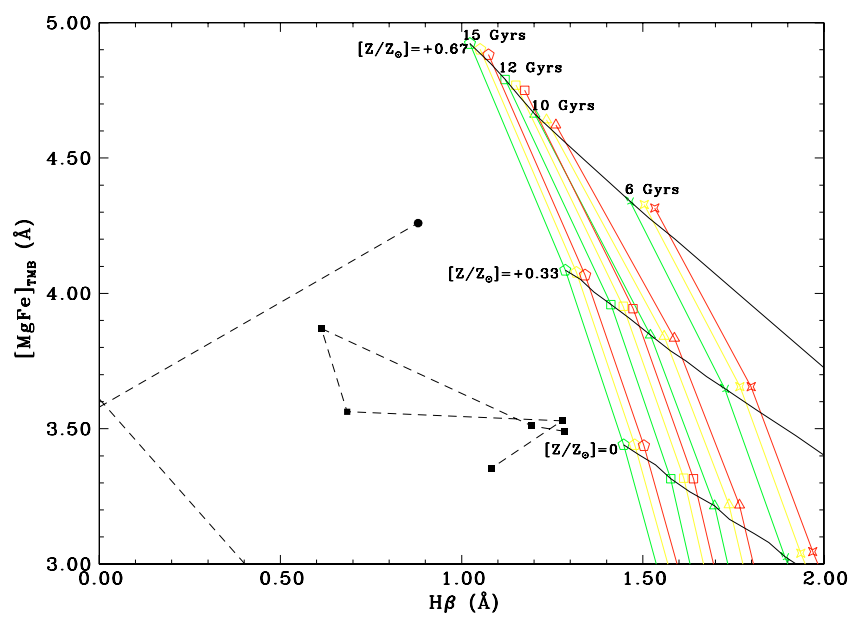

Fig. 16. $[\mathrm{MgFe}]_{\mathrm{TMB}}$ modified Lick index versus $\mathrm{H} \beta$ Lick index: comparison of Lick indices of the long slit spectra of NGC 1052 with the theoretical ones of TMB (2003a)'s SSPs. The indices of the aperture spectra are represented by solid black squares (major axis data for $6.8 \leq r \leq 20.2$ arcsec) and solid black circles (minor axis data for $6.8 \leq r \leq 18.0$ arcsec) which are connected by traced black lines as in Fig. 15. The predicted indices of the SSPs are represented by open colour symbols (ages by symbols and $[\alpha / \mathrm{Fe}]$ by colours), however the solid black lines connect the Lick indices of the SSPs with $[\alpha / \mathrm{Fe}]=0.0$ dex for each global metallicity covering different ages. In this plot, the global metallicities increase from the bottom to the top.

see Sect. 7. Along both axis directions, there is strong spread of the $\mathrm{Mg} / \mathrm{Fe}$ ratio that seems to have a monotonic radial dependency. This can be based on some inside-out galaxy formation process associated with a possible outwards radial increasing of the star formation timescale. Therefore, in NGC 7796 the chemical enrichment of $\alpha$-elements (SN-II) relative to the iron elements (SN-Ia) should occur with a radial dependency.

In the following paragraphs, the analysis in each index-index plane is given.

In the planes index vs. index, the data obtained along both axes are reasonably distributed in the same regions. For example, the data of both central apertures are close to each other.

The Mgb versus Fe4531 plot (Fig. 17a) shows the observed Lick indices of all extracted spectra with a distribution along the curve families of the SSPs with $[\alpha / \mathrm{Fe}]=0.00,+0.3$
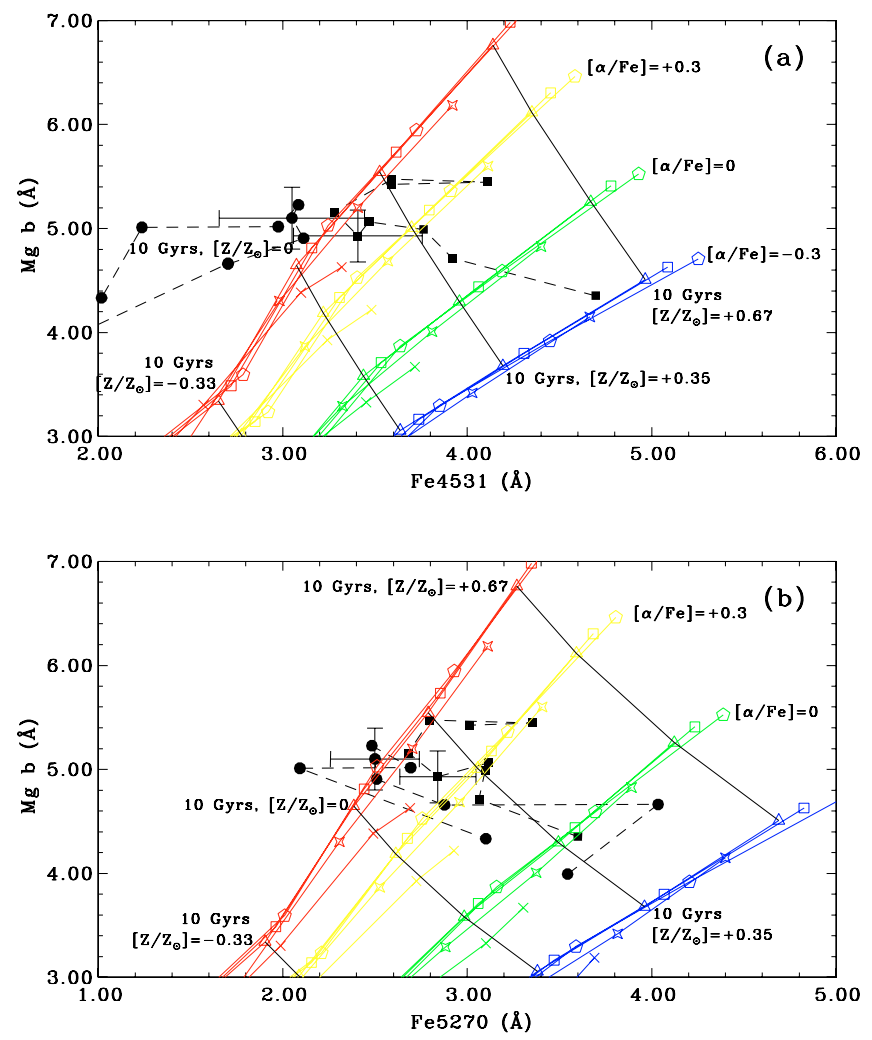

Fig. 17. a) $\mathrm{Mg}$ b versus $\mathrm{Fe} 4531$; b) $\mathrm{Mg}$ b versus Fe5270: comparisons of the observed Lick indices of the aperture spectra of NGC 7796 with the theoretical ones of TMB (2003a)'s SSPs. The notation is the same as in Fig. 11.
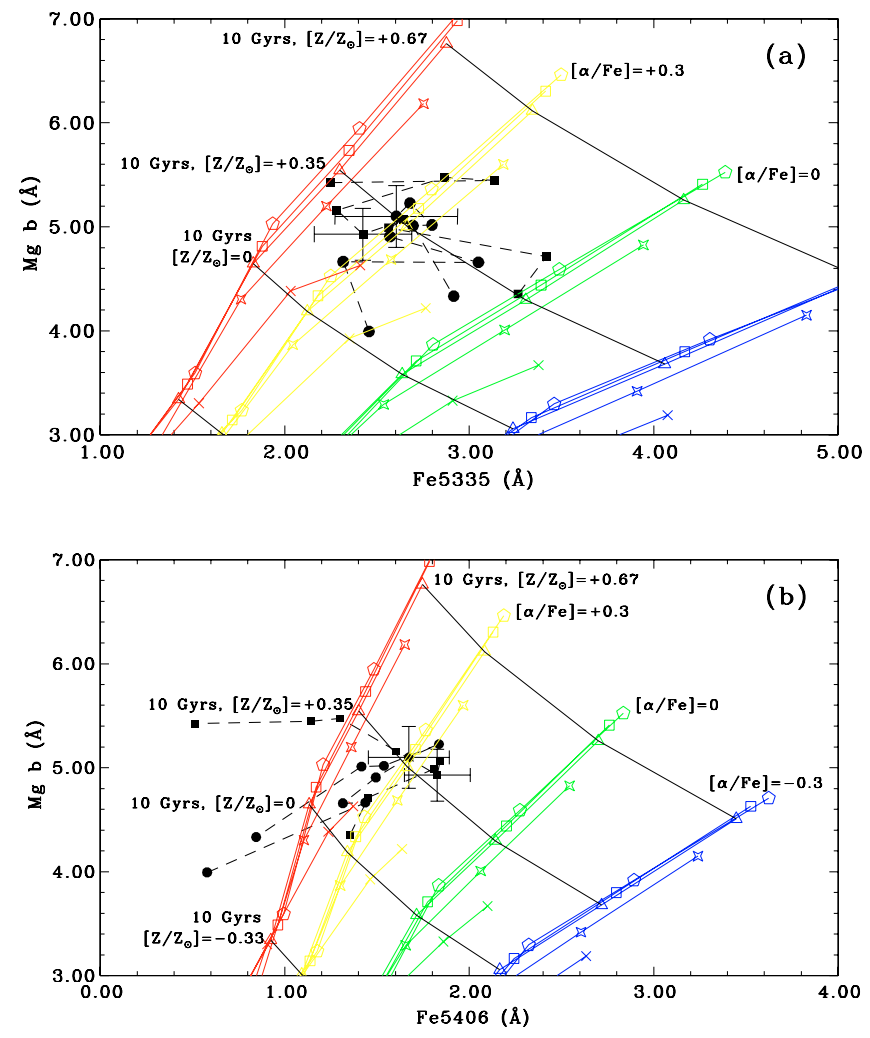

Fig. 18. a) $\mathrm{Mg}$ b versus Fe5335; b) $\mathrm{Mg}$ b versus Fe5406: comparisons of the observed Lick indices of the aperture spectra of NGC 7796 with the theoretical ones of TMB (2003a)'s SSPs. The notation is the same as in Fig. 11. 

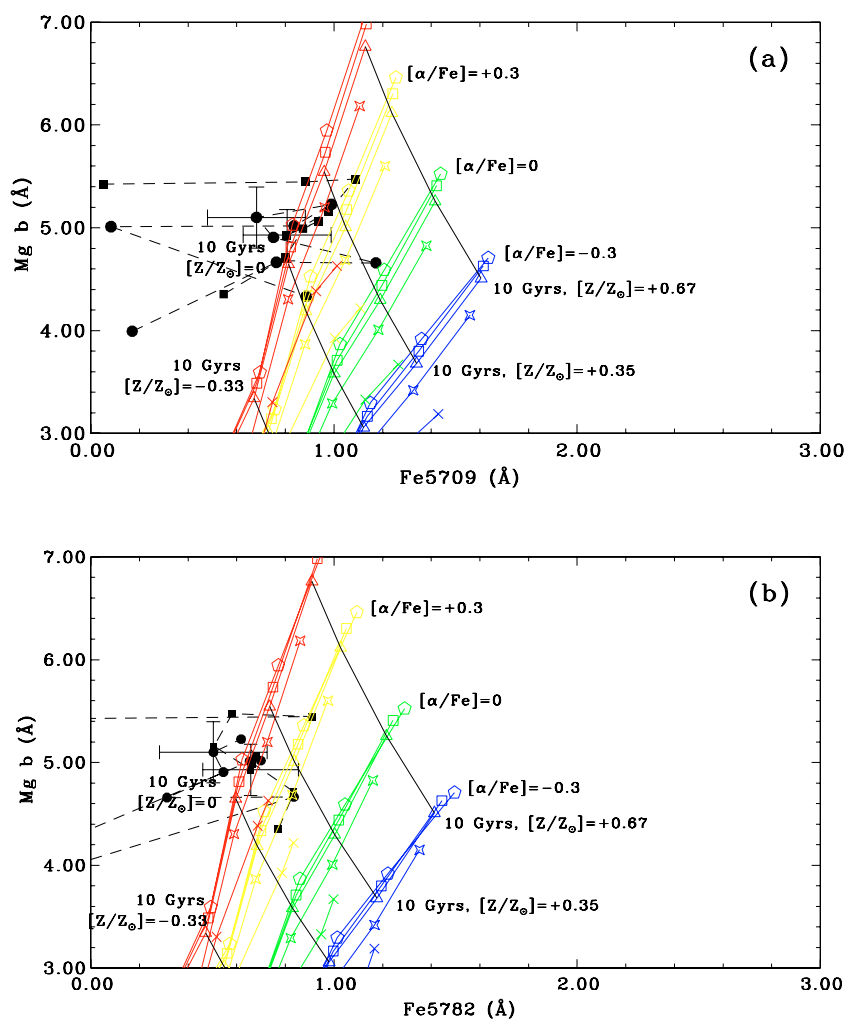

Fig. 19. a) $\mathrm{Mg}$ b versus $\mathrm{Fe} 5709$; b) $\mathrm{Mg}$ b versus $\mathrm{Fe} 5782$ : comparisons of the observed Lick indices of the aperture spectra of NGC 7796 with the theoretical ones of TMB (2003a)'s SSPs. The notation is the same as in Fig. 11.

and $+0.5 \mathrm{dex}$ in the region of the metallicities $\left[Z / Z_{\odot}\right]=0.0$ and $+0.35 \mathrm{dex}$; the data of the central spectra are localized around the curve families with highest $\alpha / \mathrm{Fe}$ ratio for the major axis data only. Some minor axis data are outside of the SSP region.

The Mgb versus Fe5270 plot (Fig. 17b) shows the indices with distributions between the curves of the SSPs with $[\alpha / \mathrm{Fe}]=$ 0.0 and $+0.5 \mathrm{dex}$ in the region of $\left[Z / Z_{\odot}\right]=0.0$ and $+0.35 \mathrm{dex}$. In this plot, the data are spread transversely to the $\alpha / \mathrm{Fe}$ family curves so that the data from the nucleus are located near the $[\alpha / \mathrm{Fe}]=+0.5$ dex curve.

The Mgb versus Fe5335 plot (Fig. 18a) shows the indices with distributions along the curves of the SSPs with $[\alpha / \mathrm{Fe}]=$ $0.0,+0.3$ and $+0.5 \mathrm{dex}$ at $\left[Z / Z_{\odot}\right]=+0.35 \mathrm{dex}$, while the data of the more central spectra are localized around the curves with higher $\alpha /$ Fe abundance ratios.

The Mgb versus Fe5406 plot (Fig. 18b) shows the indices with a distribution between the curves of the SSPs with $[\alpha / \mathrm{Fe}]=$ +0.3 and +0.5 dex in the region of $\left[Z / Z_{\odot}\right]=0.00$ and +0.35 dex.

The plots of Mgb vs. Fe5709 (Fig. 19a) Mg b vs. Fe5782 (Fig. 19b) show the indices with a distribution along the curves of the SSPs with $[\alpha / \mathrm{Fe}]=+0.3$ and +0.5 dex in the region of $\left[Z / Z_{\odot}\right]=0.00$ and +0.35 dex. Some data are outside of the SSP region in both plots.

The $\mathrm{Mg}_{1}$ versus $\langle\mathrm{Fe}\rangle$ plot (Fig. 20a) shows the indices with a distribution between the curves of the SSPs with $[\alpha / \mathrm{Fe}]=0.0$ and +0.5 dex in the $\left[Z / Z_{\odot}\right]=+0.35$ dex region.

The $\mathrm{Mg}_{2}$ versus $\langle\mathrm{Fe}\rangle$ plot (Fig. 24b) shows the indices with a distribution between the curves of the SSPs with $[\alpha / \mathrm{Fe}]=0.0$ and +0.5 dex above and in the $\left[Z / Z_{\odot}\right]=+0.35$ dex region.
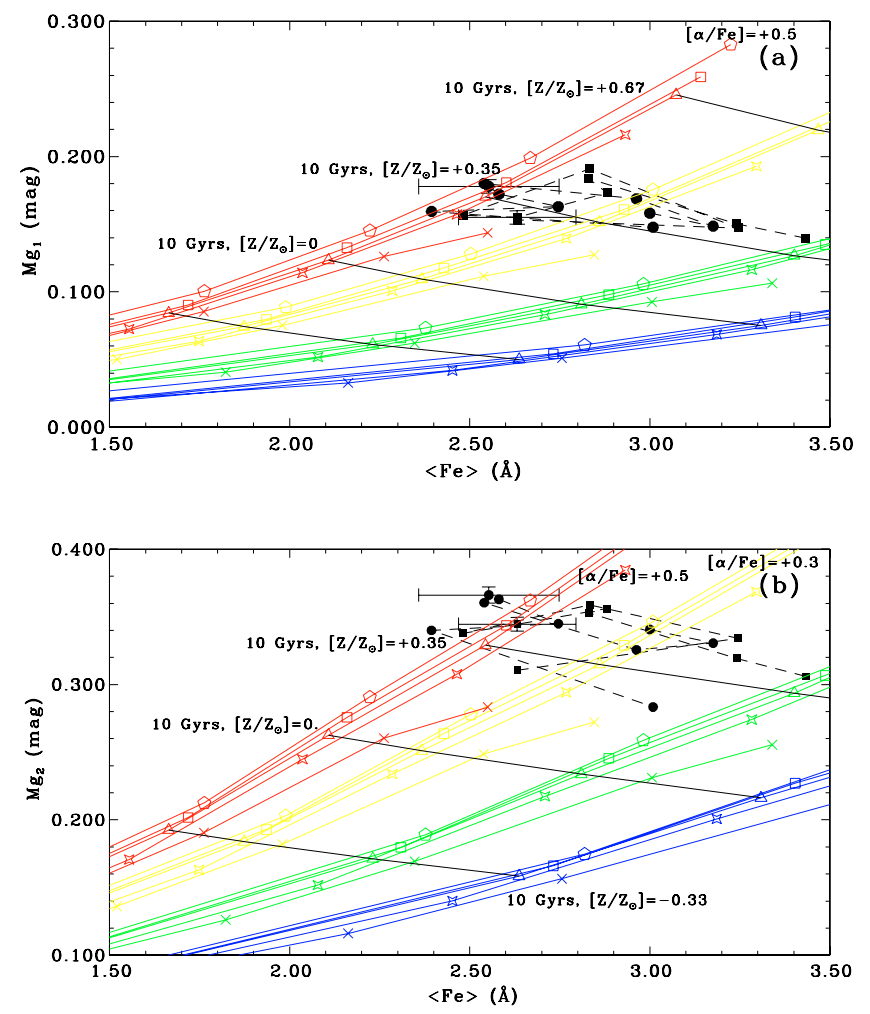

Fig. 20. a) $\mathrm{Mg}_{1}$ versus $\langle\mathrm{Fe}\rangle$; b) $\mathrm{Mg}_{2}$ versus $\langle\mathrm{Fe}\rangle$ : comparisons of the observed Lick indices of the aperture spectra of NGC7796 with the theoretical ones of TMB (2003a)'s SSPs. The notation is the same as in Fig. 11.

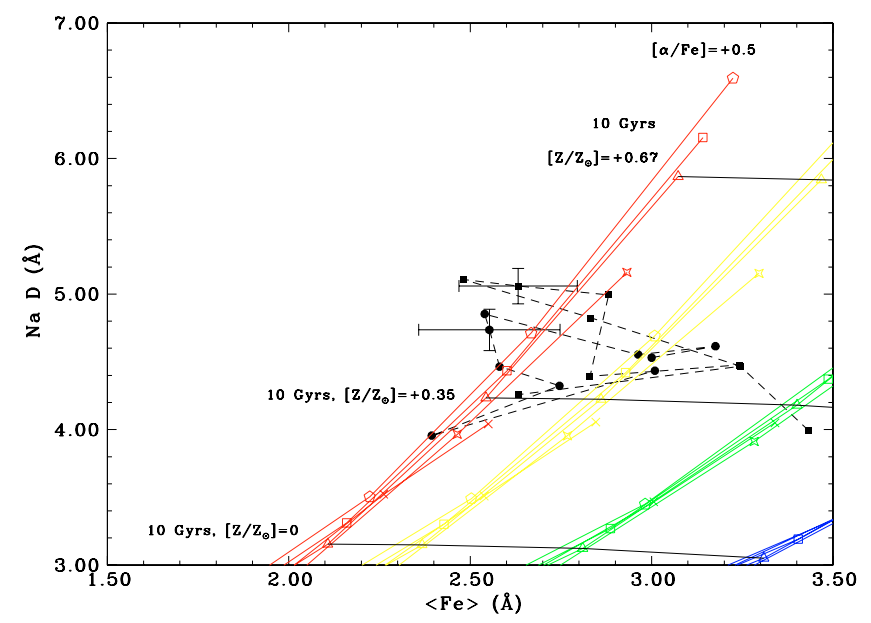

Fig. 21. $\mathrm{Na} \mathrm{D}$ versus $\langle\mathrm{Fe}\rangle$ : comparisons of the observed Lick indices of the aperture spectra of NGC 7796 with the theoretical ones of TMB (2003a)'s SSPs. The notation is the same as in Fig. 11.

Both $\mathrm{Mg}_{1}$ and $\mathrm{Mg}_{2}$ (vs. $\langle\mathrm{Fe}\rangle$ ) plots present the data distributed transversely to the $\alpha / \mathrm{Fe}$ curves so that the data from the nucleus are located near the curve of the highest $\alpha / \mathrm{Fe}$ ratio and the data of the more external apertures are near to the curves with smaller $[\alpha / \mathrm{Fe}]$.

The $\mathrm{NaD}$ versus $\langle\mathrm{Fe}\rangle$ plot (Fig. 21) shows the indices with a distribution between the curves of the SSPs with $[\alpha / \mathrm{Fe}]=0.0$ and $+0.5 \mathrm{dex}$ at $\left[Z / Z_{\odot}\right]=+0.35 \mathrm{dex}$, where the data of more spectra are localized around the curves with the highest $\alpha / \mathrm{Fe}$ ratio. However, $\mathrm{Na}$ is not an $\alpha$-element. Therefore, the overabundance of sodium relative to iron is still questionable. 


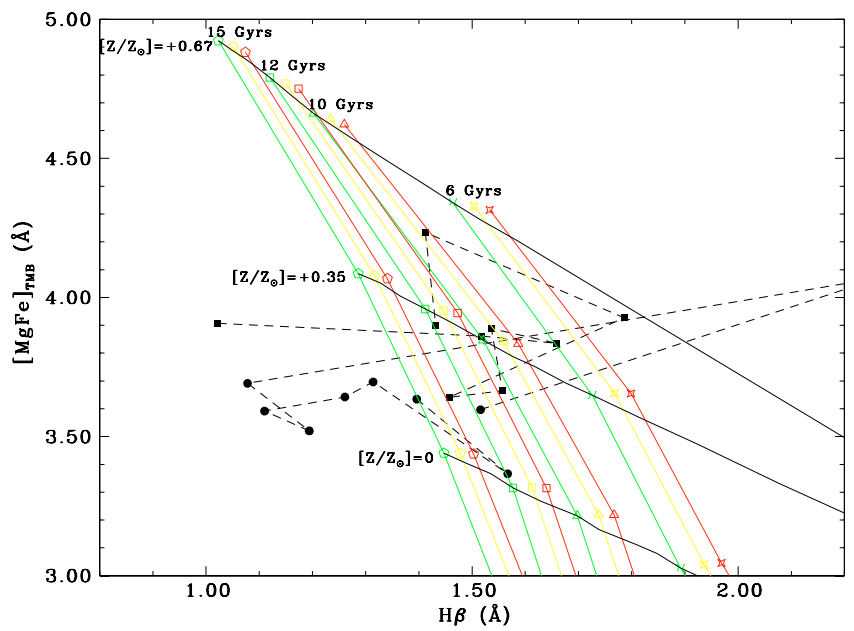

Fig. 22. $[\mathrm{MgFe}]_{\mathrm{TMB}}$ versus $\mathrm{H} \beta$ : comparison of Lick indices of the long slit spectra of NGC 7796 with the theoretical ones of TMB (2003a)'s SSPs. The notation is the same as in Fig. 16. In this plot, the global metallicities increase from the bottom to the top.

The $[\mathrm{MgFe}]_{\mathrm{TMB}}$ versus $\mathrm{H} \beta$ plot (Fig. 22) was made using all data of NGC 7796. The data of NGC 7796 are concentrated around the region of the theoretical Lick indices of the SSPs with global metallicity $\left[Z / Z_{\odot}\right]=+0.35$ dex, including the 6,10 and $12 \mathrm{Gyr}$ ages and different $[\alpha / \mathrm{Fe}]$.

The average dispersion of $[\alpha / \mathrm{Fe}]$ is nearly 0.35 dex inside the observed regions of both galaxies (the rotating disc or major axis of NGC 1052 and both main photometric directions of NGC 7796). This dispersion is still greater than the error of a single estimation of $[\alpha / \mathrm{Fe}]$, which is nearly $\pm 0.1 \mathrm{dex}$, when only one Lick index versus Lick index plot is adopted. A few plots (mainly Figs. 11a and 17a) have part of the data outside the curve grid, perhaps due to the accuracy of the Lick calibration (see Table 6 for Fe4531 and Proctor et al. 2004).

\section{Stellar population synthesis}

For a good understanding of some properties of NGC 1052 and NGC 7796, such as the presence of central gas emission, a precise determination of the star formation process is necessary. Therefore, the age of the constituent stars in these galaxies is an important parameter to be determined.

The integrated spectrum of a given galaxy contains significant information on its stellar content and chemical enrichment (Bica \& Alloin 1986a). This information together with a stellar population synthesis method is used to determine the galaxy star formation history (Bica \& Alloin 1986b). In this work we have employed the stellar population synthesis method developed by Bica (1988) which is based on integrated spectra of star clusters and $\mathrm{H}$ II regions, characterized by different ages and metallicities. In the present case, we have used seven components in the spectral base (G1, G2, G3, Y1, Y2, Y3 and RHII). The old components (G1, G2 and G3) have ages of 10, 13 and 15 Gyr and metallicities of the $\left[Z / Z_{\odot}\right] \sim 0.0, \sim-0.4$ and $\sim-1.1$ dex respectively. The components Y1, Y2 and Y3 have ages of $\sim 10, \sim 25$ and $\sim 80 \mathrm{Myr}$ with metallicities of the $\left[Z / Z_{\odot}\right] \sim-0.25, \sim-0.4$ and $\sim-0.5$ dex respectively. The HII region represents a stellar population $10^{6}$ years old and with solar metallicity.

The algorithm uses $E W$ s and continuum points measured in a given galaxy spectrum and compares them to those of a model computed from a spectral base of simple stellar population elements (see in Rickes et al. 2004).
Table 19. Equivalent widths for the spectral base.

\begin{tabular}{l|c|cccccccc}
\hline \hline \multicolumn{10}{c}{$E W(\AA)$} \\
\hline & $\begin{array}{c}\text { Metallicity } \\
{\left[\mathrm{Z} / \mathrm{Z}_{\odot}\right]}\end{array}$ & Fe4383 & $\mathrm{Ca} 4455$ & $\mathrm{Fe} 4531$ & Fe5270 & Fe5335 & Fe5406 & Fe5709 & Fe5782 \\
\hline G1 & 0.00 & 6.00 & 2.76 & 4.06 & 2.77 & 1.65 & 1.50 & 1.36 & 1.14 \\
G2 & -0.4 & 3.28 & 0.94 & 2.94 & 2.99 & 1.70 & 1.46 & 1.30 & 0.80 \\
G3 & -1.1 & 2.42 & 0.40 & 2.10 & 1.59 & 0.95 & 0.77 & 0.57 & 0.52 \\
Y1 & -0.25 & 2.73 & 1.08 & 1.10 & 1.95 & 0.70 & 1.36 & 1.22 & 0.87 \\
Y2 & -0.4 & 2.58 & 0.49 & 0.78 & 1.06 & 0.71 & 0.89 & 0.70 & 0.28 \\
Y3 & -0.5 & 1.78 & 0.57 & 1.13 & 0.88 & 0.76 & 0.53 & 0.43 & 0.33 \\
RHII & 0.00 & 0.00 & 0.00 & 0.00 & 0.00 & 0.00 & 0.00 & 0.00 & 0.00 \\
\hline
\end{tabular}

Table 20. Continuum points for the spectral base.

\begin{tabular}{c|cccccccc}
\hline \hline \multicolumn{8}{c}{$C_{\lambda} / C_{5870}$} \\
\hline & $\lambda 4475$ & $\lambda 4800$ & $\lambda 5100$ & $\lambda 5300$ & $\lambda 5546$ & $\lambda 5800$ & $\lambda 5822$ & $\lambda 6200$ \\
\hline G1 & 0.67 & 0.78 & 0.79 & 0.91 & 0.97 & 1.01 & 1.01 & 0.94 \\
G2 & 0.83 & 0.92 & 0.91 & 0.97 & 0.98 & 0.99 & 1.01 & 0.94 \\
G3 & 0.90 & 0.96 & 0.95 & 0.97 & 0.99 & 0.99 & 0.99 & 0.93 \\
Y1 & 1.16 & 1.06 & 1.04 & 1.04 & 1.12 & 1.01 & 1.03 & 0.90 \\
Y2 & 1.52 & 1.34 & 1.22 & 1.17 & 1.04 & 1.04 & 1.03 & 0.90 \\
Y3 & 1.37 & 1.29 & 1.23 & 1.08 & 1.06 & 1.00 & 0.99 & 0.81 \\
RHII & 1.87 & 1.66 & 1.45 & 1.33 & 1.21 & 1.03 & 1.00 & 0.92 \\
\hline
\end{tabular}

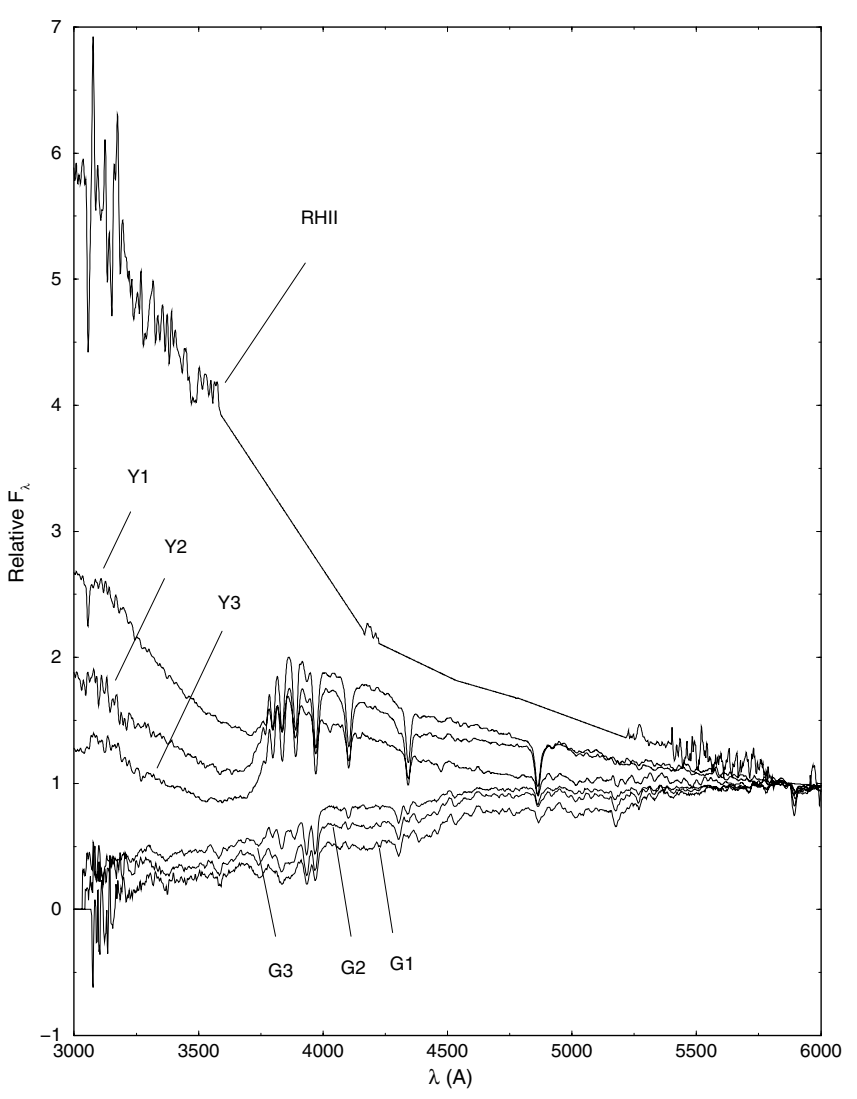

Fig. 23. Spectra of the stellar population templates used in the synthesis, normalized to $\lambda 5870 \AA$.

The corresponding selected $E W \mathrm{~s}$ and continuum points for the base elements have been measured similarly as to those for the observed galaxies, and are given in Tables 19 and 20. The base spectra are presented in Fig. 23. 
Table 21. Synthesis results in terms of flux fractions for NGC 1052 along the major axis.

\begin{tabular}{|c|c|c|c|c|c|c|c|c|}
\hline \multicolumn{9}{|c|}{ Flux fraction at $\lambda 5870 \AA$} \\
\hline$R(\operatorname{arcsec})$ & $\begin{array}{l}\mathrm{G} 1 \\
(\%)\end{array}$ & $\begin{array}{l}\mathrm{G} 2 \\
(\%)\end{array}$ & $\begin{array}{l}\text { G3 } \\
(\%)\end{array}$ & $\begin{array}{c}\mathrm{Y} 1 \\
(\%)\end{array}$ & $\begin{array}{c}\mathrm{Y} 2 \\
(\%)\end{array}$ & $\begin{array}{l}\text { Y3 } \\
(\%)\end{array}$ & $\begin{array}{l}\text { RHII } \\
(\%)\end{array}$ & $\begin{array}{c}E(B-V)_{i} \\
\quad(\mathrm{mag})\end{array}$ \\
\hline 0.00 & $61.8 \pm 8.5$ & $22.8 \pm 9.9$ & $4.2 \pm 4.4$ & $3.4 \pm 3.8$ & $3.4 \pm 3.7$ & $3.2 \pm 3.8$ & $1.4 \pm 2.3$ & 0.05 \\
\hline $1.10 \mathrm{SE}$ & $79.7 \pm 4.9$ & $3.9 \pm 4.0$ & $8.8 \pm 5.5$ & $1.4 \pm 2.5$ & $1.5 \pm 2.3$ & $3.9 \pm 3.8$ & $0.8 \pm 1.8$ & 0.00 \\
\hline $3.56 \mathrm{SE}$ & $55.0 \pm 6.1$ & $8.6 \pm 7.3$ & $5.4 \pm 5.4$ & $3.7 \pm 3.9$ & $2.4 \pm 3.1$ & $24.6 \pm 3.7$ & $0.3 \pm 1.1$ & 0.00 \\
\hline $6.84 \mathrm{SE}$ & $69.4 \pm 4.2$ & $10.9 \pm 6.9$ & $4.4 \pm 4.2$ & $0.6 \pm 1.6$ & $10.6 \pm 3.8$ & $3.5 \pm 4.1$ & $0.6 \pm 1.6$ & 0.00 \\
\hline $11.80 \mathrm{SE}$ & $57.1 \pm 5.1$ & $11.4 \pm 6.4$ & $2.6 \pm 3.5$ & $3.7 \pm 4.1$ & $20.4 \pm 5.1$ & $4.2 \pm 4.5$ & $0.4 \pm 1.4$ & 0.00 \\
\hline 20.21 SE & $24.7 \pm 4.6$ & $35.3 \pm 9.1$ & $13.7 \pm 6.7$ & $2.5 \pm 3.2$ & $6.1 \pm 4.7$ & $16.9 \pm 5.0$ & $0.7 \pm 1.7$ & 0.00 \\
\hline $1.08 \mathrm{NW}$ & $61.0 \pm 3.3$ & $19.7 \pm 3.4$ & $11.0 \pm 5.5$ & $1.0 \pm 2.0$ & $3.0 \pm 3.6$ & $3.3 \pm 4.3$ & $1.0 \pm 2.0$ & 0.04 \\
\hline $3.54 \mathrm{NW}$ & $38.6 \pm 5.1$ & $23.6 \pm 5.8$ & $18.7 \pm 4.4$ & $2.4 \pm 2.9$ & $5.4 \pm 4.0$ & $9.4 \pm 5.3$ & $2.0 \pm 2.7$ & 0.00 \\
\hline $6.81 \mathrm{NW}$ & $39.5 \pm 6.7$ & $26.9 \pm 7.0$ & $1.7 \pm 2.9$ & $7.6 \pm 5.6$ & $8.3 \pm 5.7$ & $6.4 \pm 5.2$ & $9.6 \pm 3.3$ & 0.00 \\
\hline $11.78 \mathrm{NW}$ & $32.9 \pm 4.5$ & $27.5 \pm 4.1$ & $10.4 \pm 5.6$ & $3.7 \pm 3.3$ & $8.3 \pm 2.8$ & $9.0 \pm 6.4$ & $8.1 \pm 2.8$ & 0.00 \\
\hline $20.19 \mathrm{NW}$ & $36.3 \pm 9.3$ & $29.5 \pm 9.7$ & $3.6 \pm 4.5$ & $5.9 \pm 5.4$ & $7.1 \pm 5.6$ & $6.0 \pm 6.0$ & $11.6 \pm 4.5$ & 0.00 \\
\hline
\end{tabular}

Tables notes. Last column: internal reddening, corresponding to the Galactic law.

Table 22. Synthesis results in terms of flux fractions for NGC 1052 along the minor axis.

\begin{tabular}{l|cccccccc}
\hline \hline \multicolumn{10}{c}{ Flux fraction at $\lambda 5870 \AA$} \\
\hline$R(\operatorname{arcsec})$ & $\begin{array}{c}\mathrm{G} 1 \\
(\%)\end{array}$ & $\begin{array}{c}\mathrm{G} 2 \\
(\%)\end{array}$ & $\begin{array}{c}\mathrm{G} 3 \\
(\%)\end{array}$ & $\begin{array}{c}\text { Y1 } \\
(\%)\end{array}$ & $\begin{array}{c}\text { Y2 } \\
(\%)\end{array}$ & $\begin{array}{c}\text { Y3 } \\
(\%)\end{array}$ & $\begin{array}{c}\text { RHII } \\
(\%)\end{array}$ & $\begin{array}{c}E(B-V)_{i} \\
(\mathrm{mag})\end{array}$ \\
\hline 0.00 & $60.9 \pm 7.8$ & $8.5 \pm 9.2$ & $5.1 \pm 6.1$ & $9.1 \pm 9.0$ & $11.1 \pm 7.4$ & $3.4 \pm 4.6$ & $2.0 \pm 3.2$ & 0.05 \\
\hline $2.17 \mathrm{SW}$ & $60.7 \pm 3.2$ & $1.7 \pm 2.8$ & $30.3 \pm 5.4$ & $1.0 \pm 2.0$ & $2.5 \pm 3.2$ & $3.7 \pm 3.5$ & $0.0 \pm 0.0$ & 0.02 \\
$4.90 \mathrm{SW}$ & $69.0 \pm 5.9$ & $7.3 \pm 5.7$ & $5.5 \pm 5.3$ & $3.7 \pm 4.0$ & $9.0 \pm 5.5$ & $2.7 \pm 3.4$ & $2.8 \pm 3.2$ & 0.03 \\
$9.41 \mathrm{SW}$ & $66.9 \pm 6.1$ & $13.7 \pm 6.6$ & $8.4 \pm 6.3$ & $2.2 \pm 3.1$ & $3.9 \pm 4.1$ & $3.3 \pm 3.9$ & $1.5 \pm 2.5$ & 0.02 \\
\hline $2.19 \mathrm{NE}$ & $70.3 \pm 7.3$ & $11.2 \pm 7.0$ & $9.7 \pm 6.4$ & $2.4 \pm 3.2$ & $2.3 \pm 3.1$ & $2.7 \pm 3.5$ & $1.4 \pm 2.4$ & 0.05 \\
$4.92 \mathrm{NE}$ & $57.3 \pm 8.0$ & $19.2 \pm 9.1$ & $2.7 \pm 3.9$ & $4.4 \pm 4.7$ & $9.3 \pm 6.7$ & $2.1 \pm 3.1$ & $5.0 \pm 4.0$ & 0.01 \\
$9.43 \mathrm{NE}$ & $56.5 \pm 6.6$ & $12.2 \pm 7.4$ & $4.1 \pm 4.5$ & $4.4 \pm 4.5$ & $14.5 \pm 6.8$ & $2.9 \pm 3.9$ & $5.3 \pm 3.9$ & 0.02 \\
\hline
\end{tabular}

Tables notes. Last column: internal reddening, corresponding to the Galactic law.

Table 23. Synthesis results in terms of flux fractions for NGC 7796 along the major axis.

\begin{tabular}{l|cccccccc}
\hline \hline \multicolumn{7}{c}{ Flux fraction at $\lambda 5870 \AA$} \\
\hline$R(\operatorname{arcsec})$ & G1 & G2 & G3 & $\begin{array}{c}\text { Y1 } \\
(\%)\end{array}$ & $\begin{array}{c}\text { Y2 } \\
(\%)\end{array}$ & $\begin{array}{c}\text { Y3 } \\
(\%)\end{array}$ & $\begin{array}{c}\text { RHII } \\
(\%)\end{array}$ & $\begin{array}{c}E(B-V)_{i} \\
(\mathrm{mag})\end{array}$ \\
\hline 0.00 & $54.2 \pm 6.3$ & $22.3 \pm 6.9$ & $5.9 \pm 8.1$ & $3.3 \pm 3.9$ & $4.3 \pm 4.5$ & $5.2 \pm 5.4$ & $4.6 \pm 3.8$ & 0.05 \\
\hline $1.09 \mathrm{~S}$ & $44.3 \pm 9.5$ & $29.7 \pm 9.5$ & $7.2 \pm 6.4$ & $3.9 \pm 4.4$ & $7.0 \pm 5.9$ & $2.7 \pm 3.5$ & $5.1 \pm 3.9$ & 0.04 \\
$3.55 \mathrm{~S}$ & $35.7 \pm 7.5$ & $33.0 \pm 8.7$ & $5.1 \pm 5.1$ & $7.1 \pm 4.9$ & $4.4 \pm 4.4$ & $10.2 \pm 5.2$ & $4.4 \pm 3.3$ & 0.03 \\
$7.10 \mathrm{~S}$ & $49.3 \pm 6.8$ & $22.9 \pm 7.0$ & $8.4 \pm 6.2$ & $3.3 \pm 4.0$ & $5.6 \pm 4.9$ & $7.5 \pm 5.7$ & $2.9 \pm 3.2$ & 0.01 \\
$13.21 \mathrm{~S}$ & $50.7 \pm 9.0$ & $20.8 \pm 9.5$ & $7.0 \pm 6.2$ & $7.3 \pm 5.6$ & $4.2 \pm 4.2$ & $6.5 \pm 5.6$ & $3.5 \pm 3.4$ & 0.01 \\
\hline $1.09 \mathrm{~N}$ & $51.7 \pm 9.5$ & $21.8 \pm 10.1$ & $6.0 \pm 6.2$ & $4.9 \pm 4.9$ & $7.6 \pm 5.9$ & $3.2 \pm 4.1$ & $4.8 \pm 4.0$ & 0.03 \\
$3.55 \mathrm{~N}$ & $48.0 \pm 10.6$ & $15.5 \pm 11.2$ & $12.2 \pm 8.5$ & $6.3 \pm 5.9$ & $6.0 \pm 5.2$ & $7.2 \pm 5.9$ & $4.7 \pm 3.9$ & 0.02 \\
$7.10 \mathrm{~N}$ & $43.0 \pm 10.5$ & $18.7 \pm 10.0$ & $8.1 \pm 6.9$ & $9.6 \pm 6.4$ & $6.6 \pm 5.3$ & $7.6 \pm 6.2$ & $6.3 \pm 4.5$ & 0.02 \\
$13.21 \mathrm{~N}$ & $44.6 \pm 10.5$ & $16.0 \pm 9.8$ & $9.4 \pm 7.4$ & $10.8 \pm 6.4$ & $6.7 \pm 5.2$ & $8.2 \pm 4.8$ & $4.3 \pm 3.7$ & 0.01 \\
\hline
\end{tabular}

Tables notes. Last column: internal reddening, corresponding to the Galactic law.

Table 24. Synthesis results in terms of flux fractions for NGC 7796 along the minor axis

\begin{tabular}{|c|c|c|c|c|c|c|c|c|}
\hline \multicolumn{9}{|c|}{ Flux fraction at $\lambda 5870 \AA$} \\
\hline$R(\operatorname{arcsec})$ & $\begin{array}{l}\text { G1 } \\
(\%)\end{array}$ & $\begin{array}{l}\mathrm{G} 2 \\
(\%)\end{array}$ & $\begin{array}{l}\text { G3 } \\
(\%)\end{array}$ & $\begin{array}{l}\mathrm{Y1} \\
(\%)\end{array}$ & $\begin{array}{l}\mathrm{Y} 2 \\
(\%)\end{array}$ & $\begin{array}{l}\text { Y3 } \\
(\%)\end{array}$ & $\begin{array}{c}\text { RHII } \\
(\%)\end{array}$ & $\begin{array}{c}E(B-V)_{i} \\
(\mathrm{mag})\end{array}$ \\
\hline 0.00 & $55.0 \pm 9.2$ & $21.2 \pm 9.8$ & $5.4 \pm 5.6$ & $4.4 \pm 4.7$ & $6.0 \pm 5.3$ & $4.2 \pm 4.5$ & $3.7 \pm 3.7$ & 0.02 \\
\hline $1.09 \mathrm{E}$ & $54.5 \pm 9.0$ & $25.2 \pm 9.9$ & $4.5 \pm 5.2$ & $3.4 \pm 3.6$ & $2.5 \pm 3.2$ & $3.4 \pm 3.9$ & $6.5 \pm 3.4$ & 0.01 \\
\hline $3.55 \mathrm{E}$ & $38.1 \pm 10.5$ & $23.7 \pm 11.5$ & $6.9 \pm 6.7$ & $11.1 \pm 7.2$ & $5.9 \pm 5.0$ & $10.3 \pm 6.3$ & $3.9 \pm 3.6$ & 0.01 \\
\hline $6.93 \mathrm{E}$ & $36.9 \pm 8.0$ & $24.0 \pm 9.3$ & $10.0 \pm 7.6$ & $10.6 \pm 6.1$ & $8.0 \pm 5.2$ & $7.7 \pm 5.5$ & $2.9 \pm 3.1$ & 0.02 \\
\hline $12.50 \mathrm{E}$ & $38.4 \pm 6.0$ & $21.3 \pm 8.6$ & $9.0 \pm 7.7$ & $7.00 \pm 5.8$ & $2.1 \pm 3.1$ & $20.5 \pm 6.1$ & $1.8 \pm 2.6$ & 0.01 \\
\hline $1.09 \mathrm{~W}$ & $52.2 \pm 6.7$ & $20.5 \pm 7.3$ & $5.8 \pm 5.1$ & $4.8 \pm 4.8$ & $10.9 \pm 5.1$ & $3.9 \pm 4.2$ & $1.8 \pm 2.7$ & 0.02 \\
\hline $3.55 \mathrm{~W}$ & $44.0 \pm 8.9$ & $12.7 \pm 7.9$ & $6.4 \pm 6.2$ & $7.4 \pm 5.8$ & $13.9 \pm 5.9$ & $11.6 \pm 7.1$ & $4.0 \pm 3.9$ & 0.01 \\
\hline $6.93 \mathrm{~W}$ & $54.0 \pm 8.2$ & $9.5 \pm 7.3$ & $9.0 \pm 7.3$ & $7.6 \pm 5.4$ & $10.8 \pm 5.1$ & $5.1 \pm 5.1$ & $4.0 \pm 3.8$ & 0.02 \\
\hline $12.50 \mathrm{~W}$ & $32.5 \pm 6.4$ & $37.4 \pm 8.3$ & $8.7 \pm 7.1$ & $8.5 \pm 5.9$ & $2.6 \pm 3.2$ & $4.3 \pm 4.6$ & $5.9 \pm 4.0$ & 0.03 \\
\hline
\end{tabular}

Tables notes. Last column: internal reddening, corresponding to the Galactic law. 


\subsection{Synthesis results}

The synthesis model presents the contribution of each base element to the flux at $\lambda 5870 \AA$. The computed models along the major and minor axes of NGC 1052 and NGC 7796 are given in Tables 21-24. The $E(B-V)_{\mathrm{i}}$ values are also derived from the synthesis.

For NGC 1052, the old components (G1, G2 and G3) dominate the $\lambda 5870 \AA$ flux in the more central extractions. In its nucleus, these components contribute $82 \%( \pm 10 \%)$ of the total flux (89\% for the major axis spectral extraction and $75 \%$ for the minor axis one), while at $\sim 0.3 r_{\mathrm{e}}^{\text {corr }}$ this contribution seems to decrease to $71 \%( \pm 8 \%)$ along the major axis (or the stellar disc) while it remains the same $(80 \pm 10 \%)$ along the minor axis (or the bulge). The young components (Y1, Y2 and Y3) have a small increase along the disc from the nucleus outwards: from $17 \pm 10 \%$ (center) to $25 \pm 5 \%$ (at $0.3 r_{\mathrm{e}}^{\text {corr }}$ ). Around the bulge the contribution of the young components is constant: at $0.3 r_{\mathrm{e}}^{\text {corr }}$ it is also $16 \pm 9 \%$. In terms of stellar metallicity, the synthesis results have been rebinned so that the $\mathrm{G} 1+\mathrm{Y} 1, \mathrm{G} 2+\mathrm{Y} 2+\mathrm{Y} 3$ and G3 contributions were summed in order to represent the fractions for $\left[Z / Z_{\odot}\right] \sim 0, \sim-0.4$ and $\sim-1 \mathrm{dex}$. The solar metallicity components dominate the nucleus $(68 \pm 3 \%)$ and its contribution decreases along the stellar disc $\left(49 \pm 17 \%\right.$ at $\left.0.3 r_{\mathrm{e}}^{\text {corr }}\right)$ while it remains constant around the bulge $\left(65 \pm 6 \%\right.$ at $\left.0.3 r_{\mathrm{e}}^{\text {corr }}\right)$. The fraction of the G2, Y2 and Y3 components (under-solar metallicities) is the same in the nucleus and around the bulge $(26 \pm 5 \%)$. However, it increases along the disc $\left(41 \pm 6 \%\right.$ at $\left.0.3 r_{\mathrm{e}}^{\text {corr }}\right)$. The extremely metal poor component $\mathrm{G} 3$ has a very small contribution in the observed region: $4 \pm 1 \%$ at the nucleus, $7 \pm 5 \%$ at $0.3 r_{\mathrm{e}}^{\text {corr }}$ of the minor axis (bulge) and $6 \pm 3 \%$ at $0.3 r_{\mathrm{e}}^{\text {corr }}$ of the disc. The RHII component does not show a noticeable contribution or spread. The synthesis results of NGC 1052 can be seen in Tables 21, 22 (all apertures) and Fig. 24 (nucleus, major axis observation).

The synthesis results for NGC7796 are analogous to NGC 1052. Moreover, there is more symmetry around the center for both axes than in NGC 1052. The old components dominate in the nucleus with $\sim 82.5 \pm 1 \%$ of the total flux, ( $83 \%$ for the major axis aperture and $82 \%$ for the minor axis), while at $\sim 0.3 r_{\mathrm{e}}^{\text {corr }}$ this contribution decreases to $\sim 73 \pm 3 \%$ ( $75 \pm 8 \%$ for the major axis and $71 \pm 1 \%$ for the minor one). The young components Y1, Y2 and Y3 have an outwards radial increase (14 $\pm 1 \%$, at the nucleus, to $23 \pm 4 \%$ outside). Along both axes these components increase from the central part outwards: from $13 \pm 3 \%$ to $20 \pm 6 \%$ at $0.3 r_{\mathrm{e}}^{\text {corr }}$ (major axis) and from $15 \pm 8 \%$ to $25 \pm 1 \%$ at $0.3 r_{\mathrm{e}}^{\text {corr }}$ (minor axis). In terms of stellar metallicity, the synthesis results have been rebinned in the same way as for NGC 1052. The solar metallicity components dominate the nucleus $(58 \pm 1 \%)$ and its contribution decreases along the major axis $\left(53 \pm 1 \%\right.$ at $\left.0.3 r_{\mathrm{e}}^{\text {corr }}\right)$ and minor axis $\left(54 \pm 11 \%\right.$ at $\left.0.3 r_{\mathrm{e}}^{\text {corr }}\right)$. The fraction of the undersolar metallicity component is the same in the nucleus $(31 \pm 1 \%)$ and along both axes $\left(34 \pm 2 \%\right.$ at $\left.0.3 r_{\mathrm{e}}^{\text {corr }}\right)$. The extremely metal poor component $\mathrm{G} 3$ has a very small contribution in the observed region: $5 \pm 1 \%$ at the nucleus, $8 \pm 1 \%$ at $0.3 r_{\mathrm{e}}^{\text {corr }}$ of the major axis and $9 \pm 1 \%$ at $0.3 r_{\mathrm{e}}^{\mathrm{corr}}$ of the minor axis. Therefore, the template contributions along the major axis are analogous to those along the minor axis, i.e. there is a homogeneous stellar population spatial distribution along both directions. The RHII component does not show a noticeable contribution. The synthesis results of NGC 7796 are given in Tables 23, 24 (all apertures) and Fig. 25 (nucleus, major axis observation).

The spectra representing the composite stellar population of NGC 1052 and NGC 7796 have been constructed using the star
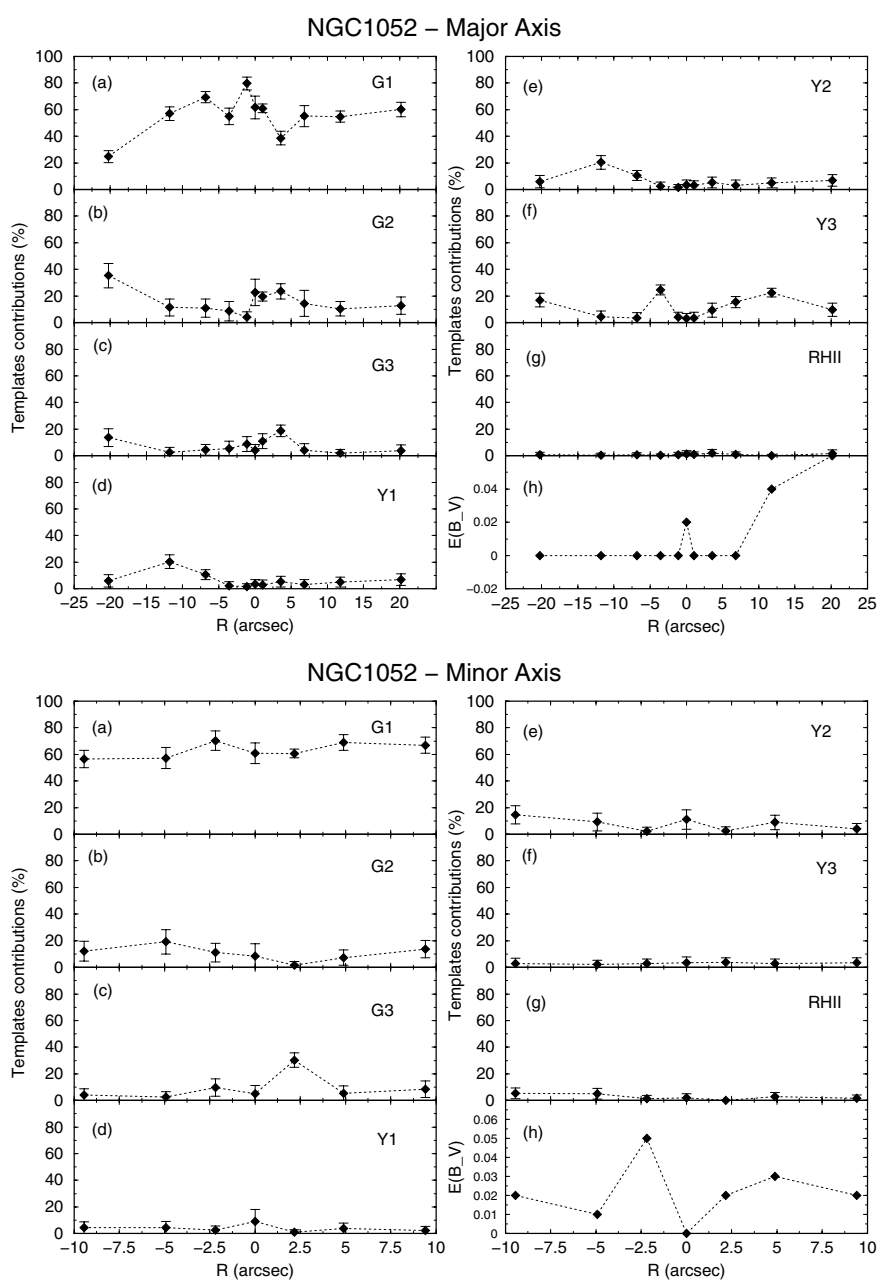

Fig. 24. Synthesis results of NGC 1052 in flux fractions as a function of the distance to the center along both photometric axes - panels a), b), c), d), e), f) and g) for the G1, G2, G3, Y1, Y2, Y3 and RHII components respectively; panel $\mathbf{h}$ ) - spatial distribution of the internal reddening. Note that the abscissa axis has a different scale for each plot.

cluster templates combined according to the proportions given by each synthesis result. We illustrate this procedure in Figs. 26 and 27 respectively for NGC 1052 and NGC 7796, in which we show the synthesis for the central aperture using their major axis extractions only (top panel). The resulting residual spectrum for each galaxy, which is obtained after the subtraction of the population template, is shown in the bottom panel of both figures. For NGC 1052, it is a pure emission spectrum with some absorption lines. For NGC 7796, it is a pure absorption spectrum. In the synthetic spectra of both galaxy nuclei compared to the observed ones, there are residuals for the $\mathrm{Mg} \mathrm{b}$ and $\mathrm{NaD}$ absorptions that are greater than for the Fe lines, possibly indicating some overabundance of those elements relative to iron.

The goodness of the stellar population synthesis method can be assessed by looking at the observed and synthetic spectra (Figs. 26 and 27).

\section{Star formation history in NGC 1052 and NGC 7796}

The star formation history of some regions of a galaxy can be characterized by the luminosity-weighted mean age of the bulk of its stars and the timescale of the corresponding main episode. In order to find the luminosity-averaged age of a galaxy region we have performed the stellar population synthesis described 

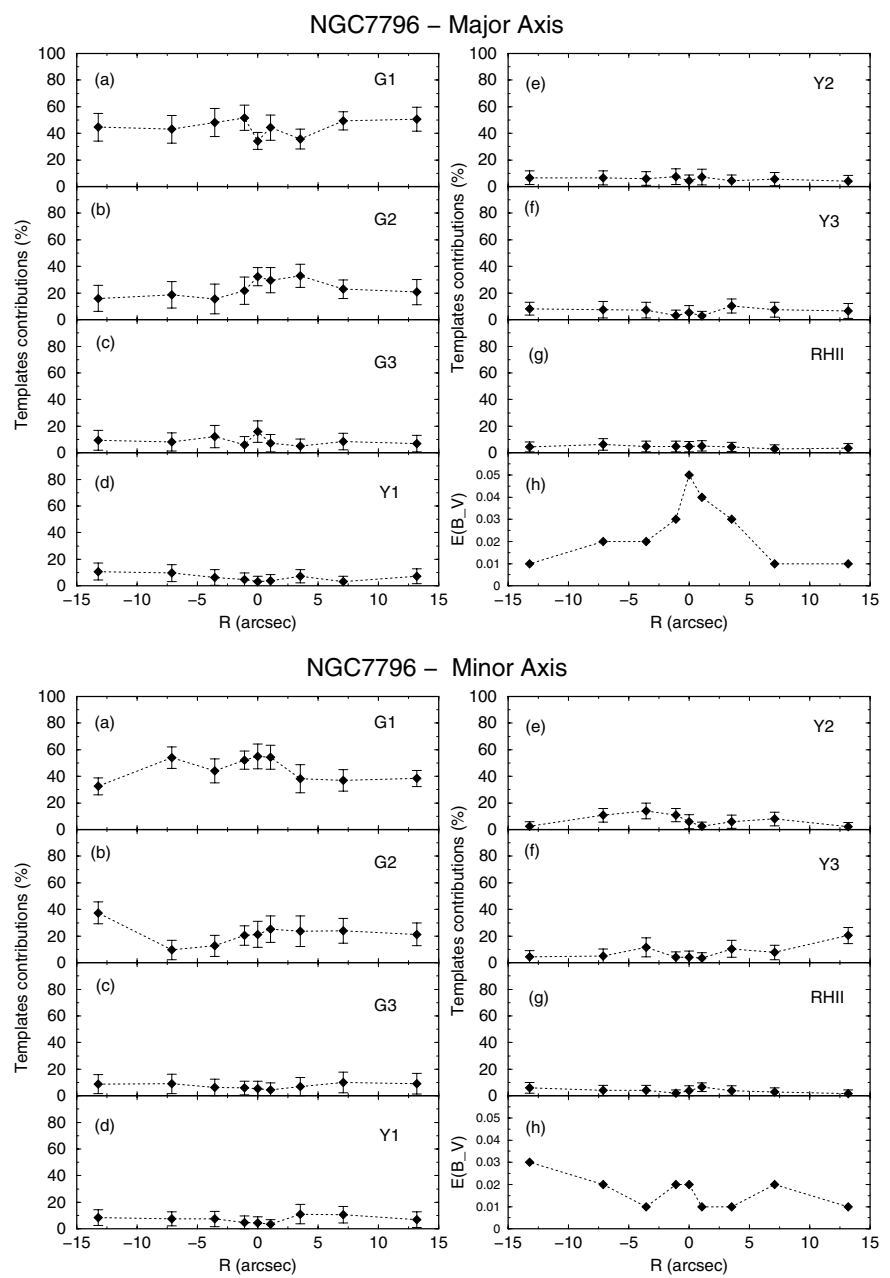

Fig. 25. Synthesis results of NGC 7796 in flux fractions as a function of the distance to the center along both photometric axes - panels a), b), c), d), e), f) and g) for each base component; panel h) - spatial distribution of the internal reddening (like Fig. 24).

in Sect. 6. The formation timescale was obtained through the the luminosity-averaged $\alpha / \mathrm{Fe}$ abundance ratio (see Sect. 5). The $[\alpha / \mathrm{Fe}]$ solar relative ratio is linearly proportional to the logarithm of the timescale of the star forming episode $\Delta t_{\mathrm{SFR}}(\mathrm{Gyr})$, which is the $F W H M$ of the Gaussian star formation rate (Thomas et al. 2005):

$[\alpha / \mathrm{Fe}] \approx \frac{1}{5}-\frac{1}{6} \log \Delta t_{\mathrm{SFR}}$.

Our results for $[\alpha / \mathrm{Fe}]$ and stellar age for NGC 1052 are divided into the bulge (minor axis data) and along the stellar disc (major axis data) as follows: (i) $\alpha / \mathrm{Fe}$ is slightly oversolar and quite homogeneous in the galaxy center $([\alpha / \mathrm{Fe}]=+0.2 \pm 0.2 \mathrm{dex})$ or around the bulge $([\alpha / \mathrm{Fe}]=+0.4 \pm 0.1 \mathrm{dex})$ but it varies along the disc from the solar value to $[\alpha / \mathrm{Fe}]=+0.5$ dex and does not show a monotonic radial dependency (we point that the average dispersion of $[\alpha / \mathrm{Fe}]$ is greater than the error of a single estimate of it, see Sect. 5); (ii) the bulk of the stars is old, 10-15 Gyr, in the bulge (dominates $82 \%$ of the $V$ light) and there is only along the disc an outwards radial increase of an intermediateaged (or young) stellar population and a small decrease of the old component; (iii) the iron abundance seems to be constant over the whole observed region applying the relation of Salaris et al. (1993) cited in Sect. 5; (iv) and the global metallicity changes

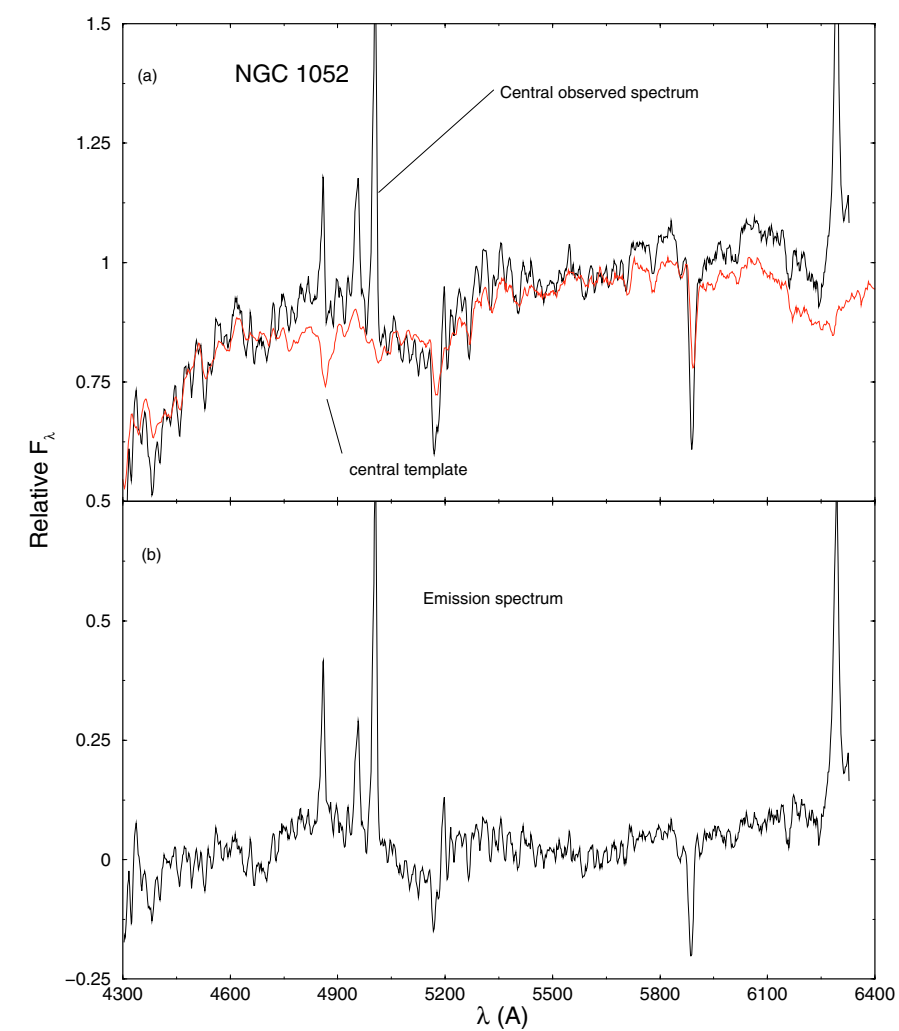

Fig. 26. Stellar population synthesis of the central extraction of NGC 1052 (major axis extraction). a) Observed spectrum corrected for reddening and template spectrum. b) Residual spectrum as an emission spectrum.

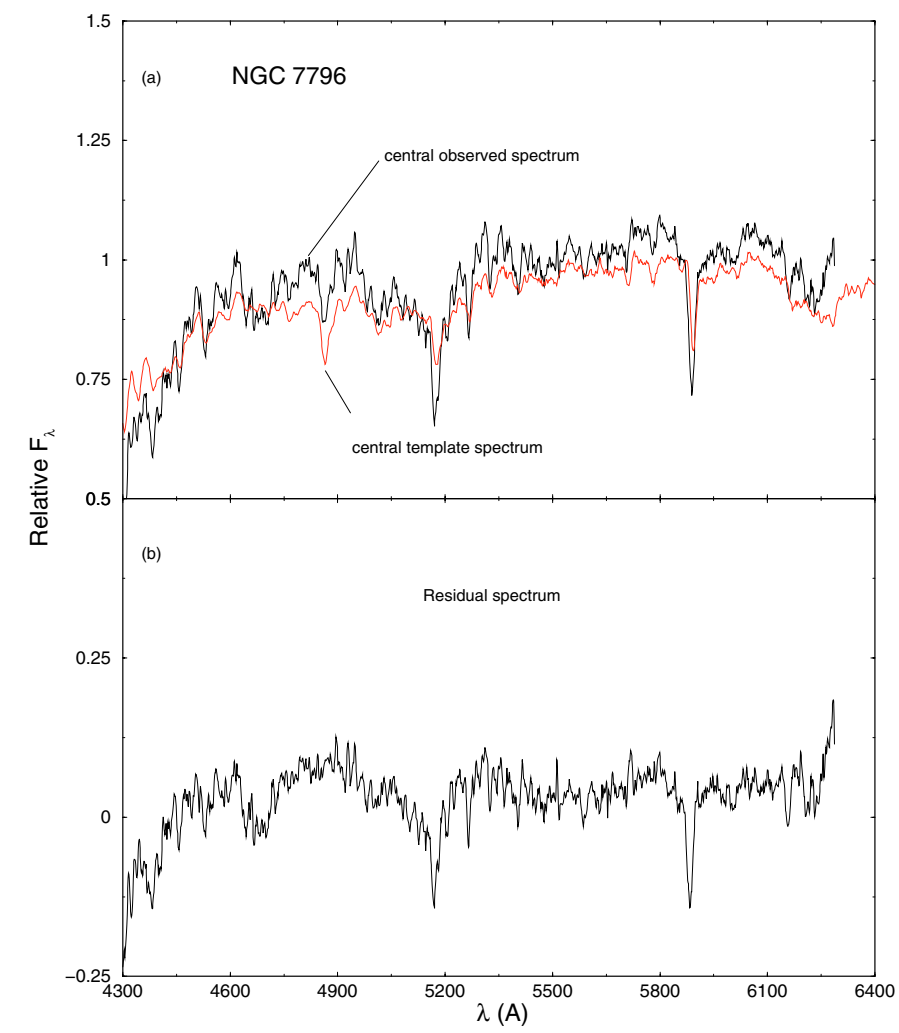

Fig. 27. Stellar population synthesis of the central extraction of NGC 7796 (major axis extraction). a) Observed spectrum corrected for reddening and template spectrum. b) Residual spectrum. 
from the solar value to $\left[Z / Z_{\odot}\right]=+0.35$ dex with a possible outwards decrease along the disc only. These results corroborate part of the conclusions of other works (Raimamm et al. 2001; Thomas et al. 2005), see Sect. 1. Therefore, the star formation history in the bulge of NGC 1052 was dominated by an ancient episode ( $\approx 13$ Gyr ago) with a timescale of 0.1 or 1 Gyr at least. The star formation timescale in the disc was heterogeoneous based on an unknown galaxy formation process.

For NGC 7796, the results for $[\alpha / \mathrm{Fe}]$ and stellar age are divided into the nucleus and along both photometric directions outwards: (i) $\alpha / \mathrm{Fe}$ is oversolar with a small dispersion in the nucleus $([\alpha / \mathrm{Fe}]=+0.4 \pm 0.1 \mathrm{dex})$ and possibly it shows a monotonic radial dependency along both axes decreasing to the solar value outwards; (ii) the stars are old in the nucleus, 10-15 Gyr (dominating $83 \%$ of the $V$ light), and there is along both directions a small outwards decreasing of this component with a small homogeneous contribution of an intermediate-aged or young component; (iii) the iron abundance seems to be constant over whole observed region if we adopt the cited relation of Salaris et al. (1993), Sect. 5; (iv) and the mean global metallicity is oversolar with a possible outwards monotonic radial decrease along both directions (or it has a high dispersion of 0.35 dex). The stellar population parameters of the nucleus agree with the results of Thomas et al. (2005); see Sect. 1. Therefore, the star formation in the center of NGC 7796 happened 10-15 Gyr ago and whose bulk of stars has a very small age spread (0.1 Gyr). The star formation must have continued outwards in order to explain the strong radial decrease of the $\alpha / \mathrm{Fe}$ ratio.

The mass of the stellar content of each galaxy has been estimated applying the relation of Thomas et al. (2005) between the stellar mass and the central velocity dispersion valid in any environment. The stellar masses of NGC 1052 and NGC 7796 are $1.4 \times 10^{11} M_{\odot}$ and $2.9 \times 10^{11} M_{\odot}$ respectively.

\section{Summary and conclusions}

We have performed a detailed stellar population analysis, adopting long slit spectroscopic observations, of two different earlytype galaxies of low density regions of the local Universe: NGC 1052, a Liner of a group that shows a stellar rotating disc, and NGC 7796, a field spheroid with a counter rotating core. Both galaxies have stellar masses around $10^{11} M_{\odot}$. The spatial distributions of the mean luminosity-weighted stellar age, metallicity, and $\alpha /$ Fe ratio along both photometric axes have been obtained in order to reconstruct the star formation history in their kinematically distinct subsystems. We have measured several Lick line-strength indices in the aperture spectra which were extracted from the galaxy center up to almost one effective radius. The Lick index radial gradients were also measured. We have confirmed the stellar kinematics of these spheroidal systems as well. The measured Lick indices were compared to the theoretical ones of the simple stellar population models of Thomas et al. (2003a) directly on the index versus index planes. We have applied a stellar population synthesis using a grid of star cluster templates from Bica (1988).

Based on the Lick index comparisons with the SSP models, both galaxies show a stellar overabundance of magnesium relative to iron, which shows a strong dispersion along the disc of NGC 1052 and is almost constant in its bulge. It is higher in the nucleus of NGC 7796 and has an outwards radial decrease along both axes. The variation of $[\alpha / \mathrm{Fe}]$ goes from 0.0 to $+0.5 \mathrm{dex}$. A plausible explanation for this is a large spread of the star formation timescale inside these galaxies during their formations. In the case of NGC 7796, the SN-Ia/SN-II ratio must increase from the galaxy center outwards in an inside-out galaxy formation process.

The results of the population synthesis indicate that the nucleus of both ellipticals is dominated by old metal rich stars $\left(\sim 13 \mathrm{Gyr}, \sim Z_{\odot}\right)$. The populations are more homogeneous in the bulge of NGC 1052 than along its disc, where there is an outwards radial decrease of the contribution of the older, richer components together with a respective rising proportion of the younger, metal poor ones. The results for NGC 7796 are analogous: there are older ages and higher metallicities in the nucleus but with a similar radial behavior of the age and $Z$ variations along both axes.

Differently from Mehlert et al. (2003), the observed regions of NGC 1052 and NGC 7796 have a strong dispersion in the parameters of their stellar populations, specially for the $\alpha /$ Fe ratio and age. Moreover, the star characteristics are associated with their kinematics: the stellar populations are older and $\alpha$-enhanced in the non-rotating bulge of NGC 1052 and counter rotating core of NGC 7796, while they show a strong spread of $\alpha / \mathrm{Fe}$ and age along the rotating disc of NGC 1052 and an outwards radial decrease outside the core of NGC 7796. The global metallicity has an outwards radial decrease, while the iron abundance is nearly constant. The age variation is possibly connected to the $\alpha / \mathrm{Fe}$.

The abundances of sodium and carbon of the stellar content seem to have some spatial dispersion in both galaxies (maybe an outwards radial decrease). However, there is likely some interstellar contribution to the $\mathrm{NaD}$ index. A careful analysis based on SSP models is still necessary because the models must consider the influence of the abundance variations of $\mathrm{Na}$ and $\mathrm{C}$ on the Lick indices.

Therefore, the bulk of the stars in the bulge of NGC 1052 and KDC of NGC 7796 was formed in an ancient short episode (maybe at 13 Gyr ago with a timescale of $0.1-1$ Gyr) providing an efficient chemical enrichment by SN-II, while in the NGC 1052 disc and outer parts of NGC 7796 the star formation occurred later with larger temporal scales, with the enrichment by SN-Ia important. Specifically, for NGC 7796 an inside-out formation is plausible, while a merging episode with a drawn out star formation is more likely for NGC 1052.

Acknowledgements. A. Milone is grateful to FAPESP, Fundação de Amparo à Pesquisa do Estado de São Paulo (process numbers 2006/05029-3 and 2000/06695-0), and Proap-Capes/INPE as well. The authors thank the referees for the suggestions and comments that improved the manuscript. The $I R A F$ (Image Reduction Analysis Facility) system was adopted for the data reduction and analysis. IRAF is distributed by the National Optical Astronomy Observatories, which are operated by the Association of Universities for Research in Astronomy, Inc., under cooperative agreement with the National Science Foundation.

\section{References}

Arimoto, N., \& Yoshii, Y. 1987, A\&A, 173, 23

Bekki, K., \& Shioya, Y. 1999, ApJ, 531, 108

Bender, R., \& Surma, P. 1992, A\&A, 258, 250

Bender, R., Doebereiner, S., \& Moellenhoff, C. 1988, A\&AS, 74, 385

Bender, R., Burstein, D., \& Faber, S. M. 1993, ApJ, 411, 153

Bertin, G., Bertola, F., Buson, L. M., et al. 1994, A\&A, 292, 381

Beuing, J., Bender, R., Mendes de Oliveira, C., Thomas, D., \& Maraston, C. 2002, A\&A 395, 431

Bica, E. 1988, A\&A, 195, 79

Bica, E., \& Alloin, D. 1986a, A\&AS, 66, 171

Bica, E., \& Alloin, D. 1986b, A\&A, 162, 21

Bica, E., Arimoto, N., \& Alloin, D. 1988, A\&A, 202, 8

Binney, J. J., Davies, R. L., \& Illingworth, G. D. 1990, ApJ, 361, 78

Burstein, D., Bertola, F., Buson, L. M., Faber, S. M., \& Lauer, T. R. 1988, ApJ, 328,440 
Cardiel, N., Gorgas, J., Cenarro, J., \& Gonzalez, J. J. 1998, A\&AS, 127, 597 Carlberg, R. G. 1984, ApJ, 286, 403

Carollo, C. M., \& Danziger, I. J. 1994, MNRAS, 270, 523

Carollo, C. M., Danziger, I. J., \& Buson, L. 1993, MNRAS, 265, 553

Chiosi, C., \& Carraro, G. 2002, MNRAS, 335, 335

Couture, J., \& Hardy, E. 1988, AJ, 96, 867

Davies, R. L., Burstein, D., Dressler, A., et al. 1987, ApJS, 64, 581

Davies, R. L., Sadler, E. M., \& Peletier, R. F. 1993, MNRAS, 262, 650

Davies, R. L., Kuntschner, H., Emsellem, E., et al. 2001, ApJ, 548, L33

de la Rosa, I. G., de Carvalho, R. R., \& Zepf, S. E. 2001, AJ, 122, 93

Duflot, M., Fehrenbach, C., Mannone, C., Burnage, R., \& Genty, V. 1995, AAS, 110,177

Faber, S. M., Wegner, G., Burstein, D., et al. 1989, ApJS, 69, 763

Ferrari, F., Pastoriza, M. G., Macchetto, F. D., et al. 2002, A\&A, 389, 355

Fried, J. W., \& Illingworth, G. D. 1994, AJ, 107, 992

Forbes, D. A., Sánchez-Blázquez, P., \& Proctor, R. 2005, MNRAS, 361, L6

Giuricin, G., Marinoni, C., Ceriani, L., \& Pisani, A. 2000, ApJ, 543, 178

Golev, V., \& Prugniel, Ph. 1998, A\&AS, 132, 255

Goudfrooij, P., \& Emsellem, E. 1996, A\&A, 305, L45

Henry, R. B. C., \& Worthey, G. 1999, PASP, 111, 919

Huchra, J. P., Brodie, J. P., Caldwell, N., Christian, C., \& Schommer, R. 1996, ApJS, 102, 29

Idiart, T. P., Michard, R., \& de Freitas Pacheco, J. A. 2003, A\&A, 398, 949

Jorgensen, I. 1997, MNRAS, 288, 161

Jorgensen, I., Franx, M., \& Kjaergaard, P. 1995, MNRAS, 276, 1341

Kadler, M., Kerp, J., Ros, E., et al. 2004, A\&A, 420, 467

Kawata, D. 1999, PASJ, 51, 931

Kobayashi, C. 2004, MNRAS, 347, 740

Kobayashi, C., \& Arimoto, N. 1999, ApJ, 527, 573

Kurtz, M. J., \& Mink, D. J. 1998, PASP, 110, 934

Larson, R. B. 1974a, MNRAS, 166, 585

Larson, R. B. 1974b, MNRAS, 169, 229

Maraston, C. 1998, MNRAS, 300, 872

Matteucci, F. 1994, A\&A, 288, 57

Matteucci, F., \& Tornambè, A. 1987, 185, 51
Matteucci, F., Ponzone, R. \& Gibson, B. K. 1998, A\&A, 335, 855

McDermid, R. M., Bacon, R. Kuntschner, H., et al. 2006, NewAR, 49, 521

Mehlert, D., Thomas, D., Saglia, R. P., Bender, R., \& Wegner, G. 2003, A\&A, 407,423

Mihos, J. C., \& Hernquist, L. 1994, ApJ, 427, 112

Morelli, L., Halliday, C., Corsini, E. M., et al. 2004, MNRAS, 354, 753

Peletier, R. F., Davies, R. L., Illingworth, G. D., Davis, L. E., \& Cawson, M. 1990, AJ, 100, 109

Pierce, M., Brodie, J. P., Forbes, D. A., et al. 2005, MNRAS, 358, 419

Pipino, A., Matteucci, F., \& Chiappini, C. 2006, ApJ, 638, 739

Proctor, R. N., Forbes, D. A., Hau, G. K. T., et al. 2004, MNRAS, 349, 1381

Raimann, D., Storchi-Bergmann, T., Bica, E., \& Alloin, D. 2001, MNRAS, 324, 1087

Ravindranath, S., Ho, L. C., Peng, C. Y., Filippenko, A. V., \& Sargent, W. L. W. 2001, AJ, 122, 653

Rembold, S. B., Pastoriza, M. G., Ducati, J. R., Rubio, M., \& Roth, M. 2002, ApJ, 588, 344

Rickes, M. G., Pastoriza, M. G., \& Bonatto, Ch. 2004, A\&A, 419, 449

Salaris, M., Chieffi, A., \& Straniero, O. 1993, ApJ, 414, 580

Saglia, R. P., Maraston, C., Thomas, D., \& Bender, R. 2002, ApJ, 579, L13

Salpeter, E. E. 1955, ApJ, 121, 161

Terlevich, R., Davies, R. L., Faber, S. M., \& Burstein, D. 1981, MNRAS, 196, 381

Thomas, D., Maraston, C., \& Bender, R. 2003a, MNRAS, 339, 897

Thomas, D., Maraston, C., \& Bender, R. 2003b, MNRAS, 343, 279

Thomas, D., Maraston, C., Bender, R., \& de Oliveira, C. M. 2005, ApJ, 621, 673

Tonry, J., \& Davis, M. 1979, AJ, 84, 1511

Trager, S. C., Worthey, G., Faber, S. M., Burstein, D., \& Gonzalez, J. J. 1998, ApJS, 116, 1

Tripicco, M. J., \& Bell, R. A. 1995, AJ, 110, 3035

Worthey, G. 1994, ApJS, 95, 107

Worthey, G., \& Ottaviani, D. L. 1997, ApJS, 111, 377

Worthey, G., Faber, S. M., \& Gonzalez, J. J. 1992, ApJ, 398, 69

Yoshii, Y., \& Arimoto, N. 1987, A\&A, 188, 13 